\title{
Qualitätsmanagement in der HNO - eine Standortbestimmung
}

\author{
Wie stellen sich bei aktueller Betrachtung die Qualitätsstandards in der \\ deutschen Krankenhauslandschaft und den Praxen, insb. der HNO-Heilkunde dar? \\ Bestandsaufnahme mit Ausblick
}

\section{Quality Management in Otolaryngology - An Assessment of the Current Situation}

\author{
On Current View, how is the State of Quality Standards in German Hospitals and \\ Practices, esp. in Otolaryngology? An Appraisal with Outlook
}

\section{(우(1) $(9)$}

Autor

Frank Wallner

Institut

Universitäts-HNO-Klinik Heidelberg

Schlüsselwörter

Qualitätsmanagement, Qualitätssicherung, Zertifizierung, Akkreditierung, deutsches Gesundheitswesen.

Key words

Quality management, quality assurance, certification,

accreditation, German health care system

Bibliografie

DOI https://doi.org/10.1055/a-1012-9442

Laryngo-Rhino-Otol 2020; 99: S336-S383

(c) Georg Thieme Verlag KG Stuttgart · New York

ISSN 0935-8943

Korrespondenzadresse

Dr. med. Frank Wallner

Univ. HNO-Klinik

Im Neuenheimer Feld 400

D-69120 Heidelberg

frank.wallner@med.uni-heidelberg.de

\section{ZUSAMMENFASSUNG}

Um mit der Unvollkommenheit ärztlichen Handelns umzugehen, gibt es neben gesellschaftlichen Sanktionen schon seit der Antike ethische Verhaltensvorgaben für Ärzte, die unser heutiges Verständnis von einem guten Arzt geformt haben. Mit Industrialisierung und Standardisierung wurde vor 100 Jahren in den USA der Grundstein für Qualitätsmanagement in der Medizin gelegt. In den 1950ern entstand in Japan ein umfassendes Qualitätskonzept, dass großen Einfluss auf die Medizin gewinnen sollte. AlleheutigenZertifizierungsund Akkreditierungsverfahren gehen aus diesen Wurzeln hervor. Seit 15 Jahren knüpft der Gesetzgeber in Deutschland ein immer feineres Netz an Regularien, um durch externe Qualitätssiche- rung Mindeststandards im Gesundheitswesen zu gewährleisten. Ärztliche Institutionen der Selbstverwaltung verbessern darüber hinaus durch eigene Initiativen die Behandlungsqualität.

Qualitätsmanagement hat dauerhaft Einzug gehalten in Krankenhäuser und Praxen, zu erkennen an Risikomanagement und Patientenorientierung. Daneben gibt es eine Vielzahl an freiwilligen ärztlichen Initiativen zur Qualitätssicherung, z. B. die Leitlinien der ärztlichen Fachgesellschaften.

Per Umfrage wurde die Implementation eines Qualitätsmanagementsystems in deutschen HNO-Kliniken und -Praxen evaluiert und mit dem Bundesdurchschnitt verglichen. Es fand sich überwiegend eine umfassendere Umsetzung als in den Vergleichsgruppen.

Die aktuellen Herausforderungen an Kliniken und Praxen sind erheblich und haben Auswirkungen auf das Qualitätsniveau. Unterfinanzierung, Personalmangel und gesellschaftliche Veränderungen sind hier genauso relevant wie Auswirkungen des wissenschaftlichen Fortschritts.

Um Qualitätsmanagement zukünftig umfassend zu implementieren, sind kluge politische Weichenstellungen und Ressourcen entscheidend - das Konzept an sich hat sich längst durchgesetzt.

\section{ABSTRACT}

To deal with medical malpractice, apart from sanctions an ethical code has been developed since ancient times which shapes our present expectation of a good physician. A century ago, industrialization and standardization initiated medical quality management in the USA. In the 1950s, the Japanese concept of total quality management arose, winning huge influence also in medicine. Every recent system of certification or accreditation originates from these roots.

In the last 15 years in Germany, minimum standards in health care have been enforced by law with increasing sophistication. Additionally, self-governed institutions of physicians have been clearly contributing to the quality of care. 
Quality management has become an integral part of the German healthcare system, most notably in risk management and patient orientation. There are also a multitude of voluntary physician-driven initiatives to improve the quality of care, among others the guidelines of the medical societies.

A survey was conducted by the author to evaluate the implementation of quality management in otolaryngological departments and practices. The degree of implementation was predominately higher than for the national peers.
Currently there are substantial challenges to the health care system which impact the quality of care. Lack of funding, shortage of qualified staff, societal changes and effects of rapid scientific progress are a few to name.

To achieve a broad implementation of quality management in the future, wise political decisions and proper funding are crucial - the concept as such has long been accepted.

\section{Inhaltsverzeichnis}

\begin{tabular}{|c|c|}
\hline & Zusammenfassung \\
\hline & Abstract \\
\hline 1. & Einleitung \\
\hline 2. & Ethik - Verpflichtung zum verantwortlichen Handeln \\
\hline 2.1 & Historische Gelöbnisse \\
\hline 2.2 & Die Genfer Deklaration des Weltärztebundes \\
\hline 2.3 & $\begin{array}{l}\text { Deklaration von Helsinki - Ethische Grundsätze für die } \\
\text { medizinische Forschung }\end{array}$ \\
\hline 2.4 & Diskussion - Der gute Arzt \\
\hline 3. & Qualitätsdefinitionen \\
\hline 3.1 & Qualität \\
\hline 3.2 & Qualitätsmanagement \\
\hline 3.3 & Qualitätssicherung \\
\hline 3.4 & PRO und Lebensqualität \\
\hline 3.5 & Qaly \\
\hline 3.6 & Diskussion - alles relativ? \\
\hline 4. & $\begin{array}{l}\text { Zertifizierungs- und Akkreditierungsmodelle (freiwillige } \\
\text { externe Qualitätssicherung) }\end{array}$ \\
\hline 4.1 & TJC - The Joint Commission \\
\hline 4.2 & ISO - International Organization for Standardization \\
\hline 4.3 & $\begin{array}{l}\text { KTQ - Kooperation für Transparenz und Qualität im } \\
\text { Gesundheitswesen }\end{array}$ \\
\hline 4.4 & EFQM - European Foundation for Quality Management \\
\hline 4.5 & Vergleich ISO, KTQ, EFQM \\
\hline 4.6 & $\begin{array}{l}\text { Zertifizierung von Praxen und MVZ - QEP, ISO, KPQM, } \\
\text { KTQ, EPA u. a }\end{array}$ \\
\hline 4.7 & Zertifizierung von Zentren \\
\hline 4.8 & Diskussion - Messbarer Nutzen? \\
\hline 5. & $\begin{array}{l}\text { Gesetzliche und institutionelle Qualitätssicherung (verpflichtende } \\
\text { externe Qualitätssicherung) }\end{array}$ \\
\hline 5.1 & Qualitätsrelevante Gesetze \\
\hline 5.2 & G-BA - Gemeinsamer Bundesausschuss \\
\hline 5.3 & IQTIG - Institut für Qualität u. Transparenz im Gesundheitswesen \\
\hline 5.4 & IQWIG - Institut für Qualität u. irtschaftlichkeit im Gesundheitswesen \\
\hline 5.5 & BÄK - Bundesärztekammer und LÄK - Landesärztekammern \\
\hline 5.6 & $\begin{array}{l}\text { AWMF - Arbeitsgemeinschaft der Wissenschaftlichen } \\
\text { Medizinischen Fachgesellschaften }\end{array}$ \\
\hline 5.7 & $\begin{array}{l}\text { KBV - Kassenärztliche Bundesvereinigung und KV - Kassenärztliche } \\
\text { Vereinigungen }\end{array}$ \\
\hline 5.8 & $\begin{array}{l}\text { DKG - Deutsche Krankenhausgesellschaft und LKG - } \\
\text { Landeskrankenhausgesellschaften }\end{array}$ \\
\hline
\end{tabular}

S336

S336

S338

S338

S338

S338

S339

\$339

S339

$\$ 339$

S340

S340

S340

S341

S341

S341

S341

S342

S344

S346

S348

S348

S349

S349

S350

S350

S351

S358

S358

S359

S361

S361
5.9 Spitzenverband-GKV und Landesverbände der Krankenkassen

5.10 MDK - Medizinischer Dienst der Krankenversicherung

5.11 SVR -Sachverständigenrat Gesundheit

5.12 Diskussion - wie Qualität messen?

6. Einrichtungsinternes und -übergreifendes Qualitätsmanagement

6.1 Führung

6.2 Qualitätsmanagementsystem

6.3 Ziele, Ergebnisse und Entwicklung

6.4 Forschung und Innovation

6.5 Prozesse

6.6 Risiko- und Fehlermanagement

6.7 Patientenorientierung

6.8 Mitarbeiterorientierung

6.9 Kommunikation, Kooperation und Interdisziplinarität

6.10 Einrichtungsübergreifende Initiativen

6.11 Diskussion - eine extensive Qualitätslandschaft

7. Stand des Qualitätsmanagements in deutschen Krankenhäusern und Praxen, insb. in der HNO-Heilkunde

7.1 Krankenhäuser - Externe Qualitätssicherung

7.2 HNO-Krankenhäuser - Ergebnisse der Umfrage

7.3 Praxen - Bericht der KBV zum Umsetzungsstand von Qualitätsmanagement

7.4 HNO-Praxen - Ergebnisse der Umfrage

7.5 Diskussion - gute Ergebnisse für die HNOHeilkunde

8. Aktuellen Herausforderungen an Qualitätssicherung und -management

8.1 Ressourcenallokation und Patientenwohl

8.2 Führung und Mitarbeiter im Krankenhaus

8.3 Forschung, Wissenschaft und Fortschritt

8.4 Qualitätsmanagement: Finanzierung überfällig - mit begleitender RCT

8.5 Externe Qualitätssicherung

8.6 Diskussion - komplexe Herausforderungen $\quad$ S376

9. Ausblick - und zunächst ein Rückblick $\$ 377$

9.1 Blick zurück $\$ 377$

9.2 Politik und Qualität $\$ 377$

9.3 Entwicklungen im Qualitätsmanagement S377

9.4 Technologie $\quad$ S377

9.5 Ärzte und Mitarbeiter $\quad$ S378

Interessenkonflikt $\quad$ S378

Literatur $\quad \$ 378$ 


\section{Einleitung}

Wenn Perfektion dem menschlichen Wesen und Handeln inhärent wäre, bräuchten wir kein Qualitätsmanagement. Die Realität ist dadurch gekennzeichnet, dass nicht nur die bestmögliche Qualität verfehlt werden kann, sondern unerwünschte Ereignisse die Gesundheit und das Leben von Patienten kosten können.

In einer weit beachteten Studie von 1991 fanden sich bei Auswertung von 30000 Krankenakten aus dem Bundesstaat New York bei 3,7\% der Patienten unerwünschte Ereignisse, von denen 13,6\% zum Tod führten. $58 \%$ der Ereignisse wurden als vermeidbar eingeschätzt, und $28 \%$ wurden auf Nachlässigkeit zurückgeführt [1]. Das Institute of Medicine extrapolierte in seinem Bericht „To Err is Human“, dass jährlich 44000 bis 98000 Todesfälle in Krankenhäusern der USA vermieden werden könnten [2]. Für Deutschland gab es für 2007 vom Aktionsbündnis Patientensicherheit (APS) die Schätzung, dass man bei stationären Patienten von 2-4\% vermeidbaren unerwünschten Ereignissen, 1 \% Behandlungsfehlern und einer konsekutiven Mortalität von 0,1 \% ausgehen muss [3]. Darüber hinaus können Mitarbeiter, interne und externe Partner, wirtschaftliche Ergebnisse und die Reputation einer Gesundheitseinrichtung von den Folgen mangelnder Qualität betroffen sein.

Als Konsequenz daraus ist ein Risiko- und Fehlermanagement heute fester Bestandteil jedes Qualitätsmanagementsystems, und wissenschaftliche Untersuchungen zu menschlichen und systemischen Fehlerursachen [4, 5] führten zu einer neuen, offeneren Kultur des Umgangs mit Fehlern.

Bei aller Unvollkommenheit haben wir aber andererseits auch eine starke positive Vision von der erreichbaren Qualität unseres Tuns, gespeist aus intrinsischer Motivation, Arbeitsethos und Ethik. Dies findet seinen Ausdruck sowohl im klinischen und forschenden Streben nach Verbesserung und Fortschritt als auch im täglichen Ringen, unter gegebenen Umständen und Einschränkungen das Optimum zu erreichen. Methoden des umfassenden Qualitätsmanagements sind dazu die Entsprechung und bieten eine systematische Unterstützung.

\section{Ethik - Verpflichtung zum verantwortli- chen Handeln}

Die Arzt-Patient-Beziehung ist wesentlich dadurch charakterisiert, dass der Patient sich mit seiner Person, seiner körperlichen Unversehrtheit, oft seinem ganzen Wohl und Wehe dem Arzt ausliefert. Dazu ist - je nach Umständen erhebliches - Vertrauen in die fachliche Kompetenz und charakterliche Integrität des Arztes erforderlich. Dieses Vertrauen zu verdienen und zu rechtfertigen ist ein ethischer Imperativ, dem nachzukommen in Selbstverpflichtungserklärungen bekräftigt wird. Die ältesten dieser Dokumente reichen an die Anfänge der schriftlichen Überlieferung der Menschheitsgeschichte zurück.

\subsection{Historische Gelöbnisse}

\subsubsection{Hippokratischer Eid}

Die wohl bekannteste Selbstverpflichtungserklärung ist der Hippokratische Eid, der dem auf der griechischen Insel Kos praktiziert habenden Arzt Hippokrates (ca. 460 bis 370 v.Chr.) zugeschrieben wird. Der Hippokratische Eid enthält bereits die wesentlichen Ele- mente der ärztlichen Ethik: Das Versprechen, Patienten nach bestem Wissen und Können zu behandeln und Schaden von ihnen abzuwenden, wird ergänzt durch das Ablehnen von Sterbehilfe und Abtreibung. Es wird gelobt, den durch die ärztliche Tätigkeit erlangten Zugang zum intimsten Lebensbereich der Patienten nicht missbräuchlich auszunutzen, insbesondere nicht durch sexuelle Handlungen. Weiterhin erfolgt eine Verpflichtung auf die ärztliche Schweigepflicht.

\subsubsection{Primum non nocere}

Eine kondensierte, auf das therapeutische Vorgehen fokussierten Handlungsanweisung, die in ihrer Prägnanz zeitlos ist, wird oft Hippokrates zugeschrieben, wurde aber möglicherweise erst im 17. Jahrhundert geprägt [6]. Insbesondere der erste Satz ist auch heutzutage im klinischen Alltag geläufig:

\section{„Primum non (nil/nihil) nocere, secundum cavere, tertium sanare“.}

Als allererste Maxime soll der Arzt dem Patienten durch sein Handeln keinen (vermeidbaren) Schaden zufügen, zweitens soll er vorsichtig und umsichtig vorgehen und erst im Anschluss daran mit seinen Heilungsbemühungen beginnen.

\subsubsection{Vejjavatapada - Indien}

Historisch aus der gleichen Epoche wie der Hippokratische Eid ist der aus Indien auf ca. 500 v. Chr. datierte Vejjavatapada überliefert. Besonders betont wurde Mitgefühl, Erbarmen und Achtsamkeit, wie es der buddhistischen Philosophie entspricht. Aus dieser Motivation heraus soll der Kranke behandelt werden, nicht aus Gewinnsucht. Der Arzt soll sich nicht entziehen, wenn seine Behandlung wirkungslos bleibt, und er soll physisch abstoßende Situationen mit Gleichmut ertragen.

\subsubsection{Konfuzianische Tradition - China}

Die chinesische Hochkultur hat, geprägt durch die konfuzianische Tradition, zur selben Zeit einen vergleichbaren Kodex für ärztliche Ethik hervorgebracht. Ärzte sollen mit Mitgefühl und ohne Ansehen des sozialen Status Patienten sorgfältig und nach bestem Können behandeln und sich dabei würdevoll verhalten. Insbesondere werden sie verpflichtet, sich mit größter Ernsthaftigkeit medizinisch fortzubilden [7].

\subsubsection{Die Siebzehn Regeln des Enjuin - Japan}

An der japanischen, buddhistischen Ri-shu Medizinschule wurde im 16. Jahrhundert für die Absolventen eine Verpflichtung mit 17 Regeln entwickelt. Als Besonderheit erfolgte die Aufforderung, Stolz und Geltungssucht zu vermeiden, die als gravierende negative Eigenschaften des Geistes angesehen werden. Stattdessen wurde Gleichmut auch undankbaren Patienten gegenüber gefordert.

\subsection{Die Genfer Deklaration des Weltärztebundes}

Die Genfer Deklaration des Weltärztebundes, die 1948 erstmalig verabschiedet wurde, liegt mittlerweile in der sechsten Revision von 2017 vor [8]. Im Gegensatz zu den historischen Gelöbnissen wurden alle religiösen Bezüge entfernt und das absolute Tötungsund Abtreibungsverbot mit einer allgemeiner gehaltenen Formu- 
lierung („höchster Respekt vor dem Leben“) relativiert. Neue Aspekte sind der explizite Vorsatz, die Autonomie des Patienten zu wahren und weiterhin die eigene Fachkompetenz (direkt oder indirekt) nicht für Repressionsmaßnahmen oder gar Folter zur Verfügung zu stellen. Ein sehr relevanter Punkt in Zeiten der kontinuierlichen Arbeitsverdichtung ist der Satz, das Ärzte auf die eigene Gesundheit und das eigene Wohlergehen gerade im Interesse der ihnen anvertrauten Patienten achten sollen. Dies war ebenfalls ein Schwerpunkt auf dem 122. Deutschen Ärztetag 2019 (s. 8.2.4).

\subsection{Deklaration von Helsinki - Ethische Grundsätze für die medizinische Forschung}

Der medizinische Fortschritt, die Weiterentwicklung des medizinischen Wissens und der medizinischen Techniken, ist mit dem Thema der medizinischen Forschung an Erkrankten oder auch Gesunden (einschließlich von Selbstversuchen) untrennbar verbunden. Die Tatsache, dass Studienteilnehmer Schaden nehmen könnten, kann nur dadurch ethisch gerechtfertigt werden, dass das Ziel die Verbesserung der Situation aller von der gleichen Gesundheitsstörung Betroffenen ist. Erste Empfehlungen zum ethischen Umgang mit medizinischer Forschung sind bereits zu Beginn des 19. Jahrhunderts dokumentiert. 1964 wurde vom Weltärztebund die Deklaration von Helsinki beschlossen, die die Rechte von Patienten, die in Studien eingeschlossen werden sollen, definiert, einen sehr elaborierten Kodex für das Design von Studien und das Verhalten forschender Ärzte enthält und Ethikkommissionen verbindlich macht [9].

\subsection{Diskussion - Der gute Arzt}

In der Zusammenschau aller oben genannten Gelöbnisse und Verpflichtungen erhält man eine auf Jahrtausenden menschlicher Erfahrung basierende Vision von einem „guten Arzt“. Da der Arzt eine zentrale Rolle im Diagnose- und Behandlungsprozess einnimmt, hat er großen Einfluss auf die medizinische Behandlungsqualität. Das Befolgen der ärztlichen Ethik ist somit die älteste, unmittelbar wirksame Qualitätssicherungsmaßnahme.

\section{Qualitätsdefinitionen}

Qualität kann als Begriff in zweierlei Hinsicht verstanden werden: erstens als Beschreiben der Beschaffenheit von etwas, und zweitens als Beschreiben der Güte von etwas. Im Kontext des medizinischen Qualitätsmanagements ist die zweite Bedeutung gemeint.

\subsection{Qualität}

Es gibt unterschiedliche Ansätze, den Begriff Qualität zu definieren, insbesondere bezogen auf das Gesundheitswesen. Qualität ist keine absolute, sondern eine relative Größe. Sie wird in der Industrie definiert als der Grad des Erreichens von Anforderungen ( = Sollwert) im Produkt (= Istwert).

\subsubsection{ISO}

In der für das Qualitätsmanagement relevanten DIN EN ISO 9000:2015, die auch im Gesundheitswesen angewendet wird, wird Qualität definiert als:

$$
\begin{aligned}
& \text { „Grad, in dem ein Satz inhärenter Merkmale eines Objekts } \\
& \text { Anforderungen erfüllt“ }
\end{aligned}
$$

In der DIN EN ISO 9001:2015 erfolgt die Definition etwas ausführlicher:

\begin{abstract}
„Vermögen einer Gesamtheit inhärenter Merkmale eines Produkts, eines Systems oder eines Prozesses zur Erfüllung von

Forderungen von Kunden und anderen interessierten Parteien“.
\end{abstract}

Bei dieser Definition erfolgt die Beurteilung der Qualität aus der Perspektive des Kunden, d. h. im Fall der Anwendung auf die Medizin in erster Linie aus der Perspektive der Patienten. Neben diesen primären Kunden können auch interne und externe Partner als Kunden kategorisiert werden. Interne Partner sind z. B. ärztliche Kollegen anderer Abteilungen, externe Partner sind z. B. Zuweiser, Kostenträger oder der Gesetzgeber. Eine sog. „Kundenorientierung“ im Qualitätsmanagement sollte deshalb alle 3 der hier genannten Gruppen einschließen.

\subsubsection{Donabedian}

Die erste bekannte Definition, die speziell auf das Gesundheitswesen bezogen ist und weite Verbreitung gefunden hat, wurde 1966 von Avedis Donabedian formuliert [10]. Er segmentierte Qualität in 3 Bereiche: die Struktur-, Prozess- und Ergebnisqualität. Dabei bezeichnet die Strukturqualität die zur Verfügung stehenden Ressourcen, die bei der Behandlung der Patienten eingesetzt werden können, was sowohl die Qualifikation, Art und Menge der Mitarbeiter als auch sämtliche technischen und baulichen Ausstattungen betrifft. Die Prozessqualität bezeichnet, wie die Leistungen am Patienten erbracht werden und umfasst dabei den gesamten Behandlungsprozess nicht nur in seinen klinischen, sondern auch administrativen Aspekten. Die Ergebnisqualität bezieht sich in erster Linie auf das Erreichen der erwarteten Behandlungsziele für den Patienten, ist darauf aber nicht beschränkt. In der praktischen Anwendung sind Struktur- und Prozessqualität leichter zu messen als die Ergebnisqualität, andererseits haben Änderungen in Struktur- und Prozessqualität nicht unbedingt eine Auswirkung auf die Ergebnisqualität. Trotz dieser Einschränkungen hat diese Definition eine weite Verbreitung im Gesundheitswesen inkl. der Gesundheitspolitik gefunden.

\subsubsection{IOM}

Eine weitere, relativ weit verbreitete Definition wurde 1990 vom „Institute of Medicine“ (IOM) publiziert [11]:

„Quality of care is the degree to which health services for individuals and populations increase the likelihood of desired health outcomes and are consistent with current professional knowledge“ (Medizinische Qualität ist der Grad, mit dem Gesundheitsdienstleistungen für Einzelne und die Gesellschaft die Wahrscheinlichkeit für erwünschte Ergebnisse erhöhen und die auf dem aktuellen Stand des fachlichen Wissens sind).

In Erweiterung der ISO-Definition wird hier explizit Bezug genommen auf den aktuellen Wissensstand des Fachgebietes. Dass der Begriff „Wahrscheinlichkeit“ - anders als in der oben genannten ISO-Definition - zwischen den „Grad“ (des Erreichens) und die „Ergebnisse“ gestellt wird, reflektiert die Schwierigkeit des Messens der Ergebnisqualität. 


\subsection{4 ÄZQ und GMDS}

Das „Ärztliche Zentrum für Qualität in der Medizin“ (ÄZQ, s. 5.5.2) zitiert folgende Definition der „Deutschen Gesellschaft für Medizinische Informatik, Biometrie und Epidemiologie“ (GMDS) [12]:

"Qualität im Gesundheitswesen bedeutet eine ausreichende und zweckmäßige, d. h. patienten- und bedarfsgerechte, an der Lebensqualität orientierte, fachlich qualifizierte, aber auch wirtschaftliche medizinische Versorgung mit dem Ziel, die Wahrscheinlichkeit erwünschter Behandlungsergebnisse bei Individuen und in der Gesamtbevölkerung zu erhöhen“.

Durch sie wird die IOM-Definition erweitert um die Spezifizierungen „ausreichend und zweckmäßig“, die synonym gesetzt werden mit „patienten- und bedarfsgerecht“, sowie "wirtschaftlich“. Dadurch werden die Kernbegriffe des $\S 12$ SGB V (s. 5.1) bezüglich der Leistungsansprüche der Versicherten eingebracht.

Es wird weiterhin ausgeführt, dass die (Qualitäts-) Anforderungen in der medizinischen Qualität unterschieden werden können in die expliziten (die vereinbarten), die impliziten (die - stillschweigend - vorausgesetzten) und die gesetzlich verpflichtenden Anforderungen [13].

\subsubsection{IQTIG}

Das „Institut für Qualität und Transparenz im Gesundheitswesen“ (IQTIG, s. 5.3) definiert Qualität in der Gesundheitsversorgung in seinen „Methodischen Grundlagen v1.1“ (S. 16, [14]) wie folgt:

"Qualität der Gesundheitsversorgung ist der Grad, in dem die Versorgung von Einzelpersonen und Populationen Anforderungen erfüllt, die patientenzentriert sind und mit professionellem Wissen übereinstimmen“.

Dabei bezieht sich das IQTIG ausdrücklich auf die Definitionen der ISO 9000:2015 und des Institute of Medicine.

\subsection{Qualitätsmanagement}

Qualitätsmanagement ist ein übergeordneter Begriff, der alle Aspekte zum Sichern und Fortentwickeln der Qualität in Bezug auf eine Organisation umfasst. Es umfasst systematische und koordinierte Maßnahmen zur Planung, Lenkung, Kontrolle und Verbesserung von Prozessen und Abläufen mit verschiedenen spezifischen Instrumenten und ist primär von der Unternehmensleitung zu verantworten, sollte aber von allen Mitarbeitern mitgetragen werden.

\subsubsection{PDCA-Zyklus nach Deming, TQM und KVP}

W. Edwards Deming war ein Mathematiker, Physiker und Statistiker, der als Wissenschaftler und Berater 1950 nach Japan eingeladen wurde, seine Ideen von Qualitätsmanagement in die Nachkriegsindustrie Japans einzubringen. Sein Konzept war äußerst erfolgreich und trug elementar zur erfolgreichen Neuaufstellung der japanischen Industrie bei [15]. Heute als „Total Quality Management“ (TQM) und „Kontinuierlicher Verbesserungsprozess“ (KVP) bekannt, propagierte er einen holistischen Blick auf die gesamte Organisation, ihre internen und externen Beziehungen, und die fortlaufende Suche nach Verbesserungsmöglichkeiten. Repräsentativ dafür ist der PDCA-Zyklus (Plan-Do-Check-Act), den er selbst seinem Lehrer Shewhart zuschrieb. Er besteht aus einem Regelkreis in 4 Schritten zur kontinuierlichen Qualitätsverbesserung:

- Plan: Es wird ein Ziel und die erforderlichen Messgrößen definiert

- Do: Umsetzung durch Maßnahmen

- Check (oder auch „Study“): Überprüfen der erreichten Ergebnisse

- Act: konsekutives Festlegen des weiteren Vorgehens

\subsubsection{Zertifizierung vs. Akkreditierung}

Diese oft synonym gebrauchten Begriffe unterscheiden sich deutlich: eine Akkreditierung stellt höhere Anforderungen als eine Zertifizierung. Die Zertifizierung bestätigt das Erfüllen von festgelegten Anforderungen, auch Konformität genannt, z. B. von Prozessen. Eine Akkreditierung stellt eine formelle Anerkennung der Kompetenz der bewerteten Organisation dar.

\subsection{Qualitätssicherung}

Im weiteren Sinne bedeutet Qualitätssicherung, dass die im Gesundheitswesen Agierenden sich durch geeignete Maßnahmen vergewissern, dass die Qualität ihrer Ergebnisse definierte Mindeststandards nicht unterschreitet. Sie hat auch den Aspekt, den Kunden dieses Einhalten von Qualitätsstandards zuzusichern, was sich in der synonymen Verwendung des Begriffs „Qualitätszusicherung“ oder auch im englischen „quality assurance“ ausdrückt. Vormalig wurde Qualitätssicherung oft synonym mit dem Begriff „Qualitätsmanagement“ benutzt. Heute versteht man sie als ein Teil des Qualitätsmanagements. Der „Gemeinsame Bundesausschuss“ (G-BA, s. 5.2) verwendet den Begriff zum Beschreiben seiner sämtlichen qualitätsbezogenen Aufgaben, insbesondere der sog. externen Qualitätssicherung.

\subsubsection{Qualitätsindikatoren}

Das IQTIG gibt folgende Definition:

„Qualitätsindikatoren sind fest definierte Kriterien, anhand deren sich medizinische Qualität in einem Krankenhaus oder in einer Praxis messen, darstellen und vergleichen lässt“ [16].

Qualitätsindikatoren sind quantitative Masse, die jedoch kein direktes Maß der Qualität. Die Validierung der in der Qualitätssicherung durch den G-BA eingesetzten Qualitätsindikatoren erfolgt durch das IQTIG.

\subsection{PRO und Lebensqualität}

PRO (patient reported outcome) wird definiert als ,jede Äußerung von Betroffenen über ihre gesundheitliche Situation und die damit in Zusammenhang stehende medizinische Behandlung “ [17]. Mit steigender Validität und Komplexität werden genannt:

- Berichte über Symptome (z. B. visuelle analoge Schmerzskalen)

- Berichte über körperliche Aktivitäten (z. B. Fähigkeit zu schlucken)

- Patientenzufriedenheit

- Patientenpräferenzen

- Lebensqualität 
Das Robert-Koch-Institut definiert die gesundheitsbezogene Lebensqualität wie folgt [18]:

"Gesundheitsbezogene Lebensqualität (Health-Related Quality of Life, HRQoL) ist ein multidimensionales „Konstrukt“ aus physischen, psychischen und sozialen Dimensionen und schließt deutlich mehr ein als lediglich Aussagen zum individuellen Gesundheitszustand. Wesentliche Orientierung ist hierbei die subjektive Wahrnehmung durch den Probanden“.

Das Messen der Lebensqualität erfolgt in aller Regel mit validierten Fragebogeninstrumenten.

Neben den klassischen Endpunkten Morbidität und Mortalität und anderen, arztbasierten Ergebnisparametern hat das PRO in den letzten Jahren bei der Bewertung der Ergebnisqualität eine deutliche Aufwertung erfahren, weil man - insbesondere mit der Lebensqualität - messen kann, „was beim Patienten ankommt“ (am Beispiel Rhinoplastik: $[19,20])$.

\subsection{Qaly}

Zur Abgrenzung davon sei noch der Qaly angeführt. Er ist ein Akronym für „Quality adjusted life years“ und ein gesundheitsökonomisches Instrument, den Nutzen von Interventionen zu quantifizieren und in Relation zu den Kosten zu setzen. Die Lebensqualität wird im Bereich von 1 (= vollständig gesund) bis 0 (= tot) bewertet. Dabei kommen die Methoden „Time Trade-off“ (wie viele Jahre Lebenserwartung wäre man bereit, für ein Rest-Leben in vollständiger Gesundheit abzugeben), „Standard Gamble“ (wieviel Todeswahrscheinlichkeit wäre man bereit in Kauf zu nehmen für vollständige Genesung) und Ratingskalen zum Einsatz. Die so erfasste Lebensqualität wird mit der Lebenserwartung in Jahren multipliziert, wodurch man den Qaly erhält. Hat eine Intervention Effekte auf Lebensqualität und/oder Lebenserwartung, ergibt sich ein veränderter Qaly. Die möglicherweise positive Differenz kann auf die Kosten der Intervention bezogen werden, d. h. man erhält einen individuellen Preis pro Jahr Qaly. Das kann für Kosten-Nutzen-Bewertungen und für Rationierungen, sei es bei knappen Therapien (Organtransplantation) oder Ressourcen, verwendet werden.

Der Qaly ist ethisch und methodisch nicht unumstritten.

\subsection{Diskussion - alles relativ?}

Aus der Gegenüberstellung dieser verschiedenen Definitionen und Herangehensweisen an den Qualitätsbegriff ergibt sich, dass je nach Perspektive und Bezugsrahmen ganz unterschiedliche Anforderungen und Messverfahren zur Evaluation verwendet werden die medizinische Ergebnisqualität ist nicht gleichzusetzen mit der Lebensqualität, der Qualitätsbegriff des Zertifizierers beinhaltet nicht das politische Gebot zur Wirtschaftlichkeit des Sozialgesetzbuches. Die Perspektive des Arztes beinhaltet unabdingbar, wie in Kapitel 2 dargestellt, die ärztliche Ethik als Kriterium für Qualität.

\section{Zertifizierungs- und Akkreditierungsmodelle (freiwillige externe Qualitätssicherung)}

\subsection{TJC - The Joint Commission}

\subsubsection{Geschichte und Organisation}

„The Joint Commission“ ist weltweit mit Abstand die älteste, gesundheitssystemspezifische Akkreditierungsorganisation.

Um die Jahrhundertwende zum 20. Jahrhundert materialisierte sich mit der sich beschleunigenden Industrialisierung das Bedürfnis nach Standardisierung in den USA in der Gründung des National Bureau of Standards im Jahr 1901 (heute National Institute of Standards and Technology, NIST). Die Vorstellungen der Ingenieure, durch Standardisierung die Effizienz zu steigern und Fehler zu reduzieren, wurde auch von ärztlicher Seite aufgenommen. Der prominente Chicagoer Chirurg Franklin H. Martin veranstaltete die ersten Chirurgiekongresse in Chicago 1910, Philadelphia 1911 und New York 1912. Letzterer wurde von 2600 Teilnehmern besucht. Auf ihm wurde eine Resolution zur „Einführung eines Systems zur Standardisierung von Krankenhäusern“ beschlossen. Gleichzeitig wurde das „American College of Surgeons“(ACS) mit dem Ziel gegründet, die Qualität der Chirurgen durch das Erteilen einer an Bedingungen geknüpften Lizenz zu gewährleisten. Ein dauerhaft eingerichtetes Komitee des ACS war das „Hospital Standardization Committee“ unter dem Vorsitz des Bostoner Chirurgen Ernest A. Codman, der ein entschiedener Vertreter der Betrachtung der $\mathrm{Er}$ gebnisqualität war („end result idea“) und der seinen Patienten sogar eine Geld-zurück-Garantie anbot. Codman konnte sich mit seinen Ideen nicht durchsetzen. Stattdessen wurde im Jahr 1917 als Ergebnis eines dreitägigen Kongresses der erste „Minimum Standard“ für Krankenhäuser verfasst. Er umfasste 5 Punkte:

1. Alle Ärzte und Chirurgen sollten als Mitarbeitergruppe organisiert sein

2. Voraussetzung zur Mitgliedschaft in dieser Gruppe sollte fachliche und ethische Kompetenz sein

3. Die Mitarbeitergruppe muss sich mindesten einmal im Monat treffen und regelmäßig ihre klinischen Erfahrungen analysieren und besprechen

4. Es müssen vollständige Krankenakten geführt werden

5. Jedes Krankenhaus muss zumindest ein klinisches Labor und eine Röntgenabteilung vorhalten

Trotz anfänglicher Schwierigkeiten waren bereits im Jahr 19207 Ärzte für das „Hospital Standardization Program“ vor Ort in den Krankenhäusern, um das Einhalten der Minimum Standards mittels einer Evaluation („Survey“) zu beurteilen. Im Jahr 1921 wurde erstmals eine Liste aller Krankenhäuser veröffentlicht, die die Standards erfüllten. In den folgenden 30 Jahren wurden die Standards weiterentwickelt und erheblich ausgedehnt und in "Standards Manuals“ zusammengefasst und veröffentlicht, um den teilnehmenden Krankenhäusern zur Vorbereitung zu dienen.

Das Programm fand weite Verbreitung und Anwendung, auch als Distinktionsfaktor für Krankenhäuser, die erfolgreich an einem Survey teilgenommen hatten. Da der Unterhalt des Programms für das ACS finanziell nicht mehr zu bewältigen war, traten 1951 weitere Organisationen in den Kreis der Gesellschafter ein und überführten das „Hospital Standardization Committee“ in die „Joint Commission on Accreditation of Hospitals“ (JCAH). Wegen der zu- 
nehmenden Kosten wurde im Jahr 1964 erstmals das Prinzip eingeführt, das Krankenhäuser für die Evaluation („Survey“ in der Terminologie der JCAH) die anteiligen Kosten übernehmen müssen. Im Jahr 1965 wurde der Medicare Act beschlossen, der die Klausel enthielt, dass Krankenhäuser, die am Medicare- und Medicaid-Programm teilnehmen wollten, durch eine JCAH-Akkreditierung ihre Qualifikation und Berechtigung nachgewiesen hätten. Dadurch erhielt die JCAH eine quasi-staatliche Funktion.

Als Folge einer Neuorientierung und Umstrukturierung wurde die Akkreditierungsmöglichkeit auch auf andere Gesundheitsorganisationen ausgedehnt und der Name 1987 in „Joint Commission on Accreditation of Healthcare Organizations“ (JCAHO) geändert. Im Jahr 1992 wurden erstmals Kriterien für die Beurteilung der Ergebnisse in das Akkreditierungsmanual aufgenommen, die 1997 durch die sog. „ORYX“-Methode zur (partiellen) Ergebnisqualitätsmessung weiter institutionalisiert wurde. Schließlich wurde im Jahr 1994 die „Joint Commission International“ (JCl) gegründet, um Akkreditierungen weltweit anbieten zu können [21]. Aufgrund von Kritik und Diskussionen über die Effektivität des Verfahrens wurde im Jahr 2006 auch die Möglichkeit von nicht-angekündigten Surveys eingeführt. Im Jahr 2007 verkürzte die JCAHO ihren Namen in „The Joint Commission“. Der Bereich der Messung der Ergebnisqualität wurde im Jahr 2015 stark überarbeitet. Knapp die Hälfte der Qualitätsindikatoren wurde gestrichen, weil die berichteten Ergebnisse konstant exzellent waren, und neue Möglichkeiten des Berichtens wurden eingeführt (electronic clinical quality measures (eCQMs), chart-abstracted measures) [22].

\subsubsection{Verfahren}

Derzeit wird das Verfahren neben Krankenhäusern auch für „Ambulatory Health Care“, „Behavioral Health Care“, „Critical Access Hospitals“, „Laboratory“, „Nursing Care Center“, „Office-Based Surgery“ und - über die Joint Commission International (JCl) - auch international angeboten.

Um akkreditiert zu werden und zu bleiben, muss sich eine Gesundheitsorganisation einem Vor-Ort-Besuch unterziehen, der spätestens alle 3 Jahre wiederholt werden muss. Üblicherweise ist der Termin im Voraus bekannt und wird in aller Regel aufwendig vorbereitet, oft sogar durch ein „Probe-Survey“. Es sind allerdings seit 2006 auch nicht-angekündigte Überraschungs-Surveys möglich.

Anhand von umfangreichen Checklisten, die auf dem veröffentlichen, aktuellen „Standards Manual“ basieren, prüft ein Team von hauptberuflichen „Surveyern“ die Konformität der Gesundheitsorganisation mit diesen Standards. Der Gesamtumfang des Surveys erstreckt sich auf 3 Bereiche: Patientenbezogene Prozesse mit Fokus auf Qualität der Versorgung und Patientensicherheit, organisationsbezogene Prozesse inkl. Führung und Risk Management, und auf die Organisationsstrukturen. Das Entdecken von sog. „Sentinel Events“, d. h. Vorfälle mit hohem Potenzial zur Patientengefährdung, kann dabei zur Abwertung oder sogar zur Verweigerung der Akkreditierung führen. Nach Abschluss des Surveys kann die Gesundheitsorganisaton entweder eine Akkreditierung mit Auszeichnung, eine reguläre Akkreditierung, eine Akkreditierung mit Typ 1 Empfehlungen (Aufträge zur Nachbesserung ohne Einschränkung der erteilten Akkreditierung), eine bedingte Akkreditierung, eine einstweilige Akkreditierung, eine vorläufige Akkreditierung oder keine Akkreditierung erhalten [23]. Im letzteren Fall ist ein
Folge-Survey frühestens ein Jahr später möglich, in den 3 davor genannten Fällen müssen erfolgreiche Nachbesserungen nachgewiesen werden. Ergänzend zu den Surveys legt die Joint Kommission in den letzten Jahren eine hohe Priorität auf die jährliche, elektronische Übermittlung von Qualitätsindikatoren durch Krankenhäuser (s. 4.1.1).

Aktuell hat die Joint Commission ihren Fokus auf die Patientensicherheit verstärkt und greift mit der visionären Kampagne „Leading the way to ZERO“ (harm), die das vollständige Vermeiden von unerwünschten Ereignissen zum Ziel hat, das Motiv des „primum non nocere" auf [24].

Parallel zum Akkreditierungsprogramm hat die Joint Kommission seit 2005 zusätzlich ein umfangreiches Zertifizierungsprogramm aufgebaut, das neben Disease-Management-Programmen auch für „Comprehensive Cardiac Center“, „Health Care Staffing Services“, „Integrated Care“, „Palliative Care“, „Perinatal Care“, „Primary Care Medical Home“, „Patient Blood Management“ sowie international angeboten wird.

\subsubsection{Relevanz}

In den USA ist die Joint Commission mit mehr als 20000 akkreditierten Gesundheitseinrichtungen mit weitem Abstand der Marktführer, und ihre internationale Tochter JCl hat weltweit $939 \mathrm{Kran}$ kenhäuser in 66 Ländern zertifiziert (Stand 2017) [25]. Nur in Deutschland ist durch die Präsenz der KTQ (s. 4.3) das Marktsegment, das sonst von der JCl bedient wird, bereits besetzt. Wohl aus diesem Grund sind in Deutschland gegenwärtig nur 2 Krankenhäuser nach $\mathrm{JCl}$ zertifiziert [26].

\subsection{ISO - International Organization for Standardiza- tion}

\subsubsection{Geschichte und Organisation}

Die ISO (International Organization for Standardization) ist weltweit der größte Entwickler für internationale Standards. Auf einer Konferenz von 25 nationalen Normungsorganisationen, die im Oktober 1946 in London stattfand, wurde der Beschluss zur Gründung der ISO gefasst, in der ähnliche Vorläuferorganisationen aufgehen sollten. Da das Akronym für die International Organization for Standardization in verschiedenen Sprachen unterschiedlich ausfällt, einigte man sich darauf, das griechische Wort „Isos“ (dt. „gleich“) als Grundlage der Namensgebung zu verwenden.

Als unabhängige Nicht-Regierungsorganisation ohne Gewinnerzielungsabsicht nahm sie 1947 mit ihrem zentralen Büro in Genf ihre Tätigkeit auf. Seitdem hat sie 22729 internationale Standards veröffentlicht und hat mittlerweile Mitglieder aus 164 Ländern. Insgesamt 783 technische Komitees und Subkomitees entwickeln die Standards. Deutschland ist seit 1951 durch das Deutsche Institut für Normung (DIN) als Mitglied in der ISO vertreten. Von der DIN in Deutschland adaptierte Normen werden als DIN EN (für Europäische Norm) ISO bezeichnet [27].

\subsubsection{Verfahren}

Für das Qualitätsmanagement in Gesundheitsorganisationen kommen die Normen der DIN EN ISO 9000 - Familie zur Anwendung. Sie wird weltweit von mehr als einer Million Organisationen angewendet. Die letzte Revision der ISO 9001 Norm fand im Jahr 2015 statt, was sich im Zusatz der Jahreszahl zur Norm ausdrückt: DIN EN ISO 9001:2015. 
Von den vier Normen der 9000er-Familie ist die ISO 9001 diejenige, die für den eigentlichen Zertifizierungsprozess Anwendung findet und in der die Anforderungen an ein Qualitätsmanagementsystem festgelegt sind. In der DIN EN ISO 9000:2015 werden Grundlagen und Begrifflichkeiten erläutert. Die DIN EN ISO 9004: 2012 stellt einen Leitfaden für die Entwicklung eines effizienten und umfassenden Qualitätsmanagementsystems dar und ist nicht die Grundlage für eine Zertifizierung, sondern versucht, das Konzept des Total Quality Management (TQM) in das ISO-Universum zu integrieren. Dadurch findet eine noch stärkere Annäherung an Exzellenzmodelle wie EFQM statt (s. 4.4.2). Schließlich gibt es noch die DIN EN ISO 19011:2018, in der der eigentliche Zertifizierungsprozess von Managementsystemen, der in der Terminologie der ISO „Audit“ genannt wird, genau beschrieben und festgelegt wird [28]. Die früher bestehenden Normen ISO 9002 und ISO 9003 wurden wegen Obsoleszenz zurückgezogen und ihr Inhalt in die anderen Normen integriert, soweit erforderlich.

\subsubsection{Die DIN EN ISO 9001:2015}

Bei der letzten Überarbeitung der ISO 9001 wurde die Zahl der Kapitel auf 10 erhöht. Zum einen wurde ein neues Kapitel „6. Planung für das Qualitätsmanagementsystem“ eingeführt, und das bisherige Kapitel 8 wurde aufgeteilt in „9. Bewertung der Leistung“ und „10. Verbesserung“.

Die ersten 3 einleitenden Kapitel dienen der Erörterung: 1. Anwendungsbereich, 2. Normative Verweise und 3. Begriffe. Die weiteren Kapitel der Norm orientieren sich am PDCA-Zyklus nach Deming. Die nächsten 3 Kapitel entsprechen dem „Plan“: 4. Kontext der Organisation, 5. Führung und 6. Planung für das Qualitätsmanagementsystem. Die 2 nächsten Kapitel entsprechen dem „Do“: 7. Unterstützung und 8. Betrieb (Operation). Das vorletzte Kapitel entspricht dem „Check“: 9. Bewertung der Leistung. Das letzte Kapitel entspricht dem „Act“: 10. Verbesserung.

Als grundsätzliche Prinzipien des Qualitätsmanagements werden genannt:

- Kundenorientierung

- Führung

- Mitarbeiter einbeziehen

- Prozessorientierung

- Kontinuierliche Verbesserung

- Evidenz-basierte Entscheidungsfindung

- Kommunikation und Kooperation

Zusammenfassend kann man sagen, dass das ISO-System zusätzlich zur klaren Prozessorientierung das Ziel der kontinuierlichen Verbesserung durch Einbeziehen der Ergebnisbewertung hat, insbesondere durch die Ausrichtung am PDCA-Zyklus. Neben dem starken Fokus auf die Kundenbedürfnisse hat der Bereich RisikoManagement eine zentrale Bedeutung erhalten [29].

\subsubsection{Audit und Zertifizierung}

Schon immer bestand nach ISO die Möglichkeit, neben Gesamtorganisationen auch Teilorganisationen wie z. B. eine Klinik in einem Klinikum oder ein Labor zu zertifizieren.

Als ersten Schritt muss die Organisation ein Qualitätsmanagementsystem aufbauen, das an der ISO 9001:2015 orientiert sein sollte, und ein QM-Handbuch erstellen. Es ist sinnvoll, dazu eigene
Mitarbeiter schulen zu lassen. Dann muss eine Selbstbewertung durch eine interne Evaluation nach ISO-Kriterien, ein sog. „Audit“, sowie verschiedene Messungen und Erhebungen (z. B. über Patientenzufriedenheit) durchgeführt werden und im „Managementreview“ dokumentiert werden. Die Ergebnisse dieser Selbstbewertung müssen in Relation zu formulierten Zielen gesetzt werden.

Anschließend wird mit einer externen Zertifizierungsgesellschaft, die von der Deutschen Akkreditierungsstelle (DAkkS, s. 4.2.3) akkreditiert sein muss, eine Zertifizierung vertraglich vereinbart. Empfehlenswert, aber nicht verpflichtend ist ein sog. VorAudit, bei dem Verbesserungsbereiche identifiziert und bis zum eigentlichen Audit nachgebessert werden können. Das eigentliche Audit, die Fremdbewertung, ist in 2 Stufen unterteilt, wobei von den Auditoren in der ersten Stufe die Dokumentation und das Management bewertet werden, und in der zweiten Stufe an den $\mathrm{Ar}$ beitsplätzen der Mitarbeiter die Umsetzung des Qualitätsmanagementsystem überprüft wird. Je nach Größe der Organisation kann das Audit einen oder mehrere Tage dauern. Als Ergebnis des Audits wird ein Auditbericht erstellt. Wenn Konformität zur Norm festgestellt wurde, erhält die Organisation das für 3 Jahre gültige Zertifikat. Wenn die Konformität wegen Abweichungen unterschiedlichen Ausmaßes zunächst nicht gegeben ist, werden Korrekturmaßnahmen vereinbart, deren Umsetzung vom Auditor im Nachgang kontrolliert wird und von dem im positiven Fall anschließend die Zertifikatsvergabe initiiert wird. Sollten wesentliche Mängel festgestellt worden sein, wird kein Zertifikat erteilt. Bei erteiltem Zertifikat ist es verpflichtend, jährlich sog. Überwachungsaudits durch externe Auditoren durchführen zu lassen, die die Aufrechterhaltung des Qualitätsmanagementsystems überprüfen. Nach 3 Jahren erfolgt auf Wunsch die Re-Zertifizierung.

\subsubsection{DAkkS - Deutsche Akkreditierungsstelle}

Die DAkkS ist die nationale Akkreditierungsstelle der Bundesrepublik Deutschland. Sie ist eine privatwirtschaftliche Institution, die auf Grundlage einer EU-Verordnung, dass nur eine Akkreditierungsstelle pro Staat zulässig ist, gegründet wurde und im Jahr 2010 ihre Tätigkeit aufnahm. Durch einen sog. Beleihungsvorgang des Bundes ist die DAkkS autorisiert, hoheitliche Akkreditierungstätigkeiten wahrzunehmen. Anteileigner sind zu je einem Drittel der Bund, die Länder und der Bundesverband der Deutschen Industrie (BDI). Die DAkkS untersteht der Aufsicht des Bundesministeriums für Wirtschaft und Energie (BMWi).

Die Aufgabe der DAkkS ist, Konformitätsbewertungsstellen (Laboratorien, Inspektions- und Zertifizierungsstellen) darauf zu prüfen, ob sie „ihre Aufgaben fachkundig und nach geltenden Anforderungen erfüllen. Kurz: Die DAkkS prüft die Prüfer “ [30]. Bei positivem Ergebnis der Prüfung wird die Konformitätsbewertungsstelle akkreditiert. Der Nachweis der Akkreditierung ist in der Praxis unabdingbar für die Geschäftstätigkeit einer Konformitätsbewertungsstelle.

Akkreditiert werden können u. a. Laboratorien (Prüf- und Kalibrierlaboratorien, Medizinische Laboratorien), Zertifizierungsstellen (für Personen, Managementsysteme und für Produkte, Prozesse und Dienstleistungen), Anbieter von Eignungsprüfungen (Ringversuchsanbieter).

Von Verbänden der zu akkreditierenden Konformitätsbewertungsstellen wurden in den letzten Jahren deutliche Kritik an der DAkkS formuliert. Die Akkreditierungsvorgänge seien bürokratisch 
überfrachtet, die Bearbeitungsdauern unakzeptabel lang und die Preise seinen überhöht. Das stelle gerade für kleine und mittelständige Unternehmen erhebliche Belastungen dar [31-33].

\subsubsection{Weiterentwicklung des Modells}

Das ISO-Modell hat sich in den vergangenen Jahren deutlich weiterentwickelt. Galt vor 25 Jahren noch der „Running Gag“, mit ISO könne man wegen der reinen Prozessorientierung auch „Schwimmwesten aus Beton" erfolgreich zertifizieren, so hat sich das insbesondere in der ISO 9001 mit der starken Kunden- und Ergebnisorientierung und der Annäherung an Exzellenzsysteme wie EFQM drastisch geändert.

\subsubsection{Relevanz}

Die ISO veröffentlicht keine Daten zur Anzahl der nach ihren Standards zertifizierten Gesundheitseinrichtungen. Beratungsfirmen gehen davon aus, dass ISO in den letzten Jahren KTQ (s. 4.3) überflügelt hat und derzeit das am weitesten verbreitete Zertifizierungssystem in den Krankenhäusern Deutschlands ist [34]. In den Praxen liegt ISO auf dem zweiten Platz hinter den von den Kassenärztlichen Vereinigungen herausgegebenen Systemen (s. 4.6.2)

\subsection{KTQ - Kooperation für Transparenz und Qualität im Gesundheitswesen}

\subsubsection{Geschichte und Organisation}

Die „Kooperation für Transparenz und Qualität im Gesundheitswesen“ GmbH (KTQ) wurde 1997 als Machbarkeitsstudie mit einer Anschubfinanzierung durch das Bundesgesundheitsministerium begonnen. Im Jahr 2001 wurde die „Kooperation für Transparenz und Qualität im Krankenhaus gGmbH“ gegründet. Gesellschafter waren ursprünglich die Bundesärztekammer (BÄK), der Deutsche Pflegerat (DPR), die Deutsche Krankenhausgesellschaft (DKG), und die gesetzlichen Krankenkassen. Das Ziel war, ein auf die Bedürfnisse und Spezifika des (deutschen) Gesundheitswesens zugeschnittenes Zertifizierungsverfahren zu entwickeln, um die Krankenhäuser auf die kommende Verpflichtung zum internen Qualitätsmanagement vorzubereiten (s. 5.1). Im Jahr 2002 begann der Regelbetrieb und die ersten Zertifizierungen wurden durchgeführt. Die Art der zertifizierbaren Einrichtungen wurde schrittweise ausgedehnt. Derzeit können neben Krankenhäusern auch Rehabilitationseinrichtungen, Praxen und MVZs, Pflegeeinrichtungen (ambulant und (teil-) stationär) und Hospize, Rettungsdienste, und seit Katalog-Version 6.0 auch mehrere zusammenhängende Einrichtungen der gleichen Art (Verbundzertifizierung), mehrere zusammenhängende Einrichtungen unterschiedlicher Art (Vernetzte Zertifizierung) sowie Organisationseinheiten, d. h. Teileinrichtungen in großen Organisationen, zertifiziert werden. Letzteres war über viele Jahre ein Hauptkritikpunkt am KTQ-Verfahren, da vorher nur z. B. ein gesamtes Universitätsklinikum, aber nicht eine einzelne Klinik zertifiziert werden konnte. Dies wurde als Vorteil des ISOVerfahrens angesehen, bei dem die Zertifizierung von Teileinheiten schon immer möglich war.

Im Jahr 2017 traten die gesetzlichen Krankenkassen aus dem Kreis der Gesellschafter aus, im Jahr 2019 auch die restlichen ursprünglichen Gesellschafter. Die Anteile wurden an die mittlerweile gegründete KTQ International $\mathrm{GmbH}$ übertragen, über die die
Aktivitäten ins (zunächst deutschsprachige) Ausland ausgedehnt werden sollen.

\subsubsection{Verfahren}

Die KTQ-Zertifizierung hat eine eindeutige Prozessorientierung: ihr Ziel „war und ist stets die Optimierung von Prozessen innerhalb der Patientenversorgung“, und der Zertifizierungsvorgang dient dazu, „Aussagen über die Qualität der Prozessabläufe in der Versorgung treffen zu können “ [35].

Das KTQ-Modell wird in den KTQ-Katalogen spezifiziert, die bezogen auf die Einrichtungsart angepasst sind. Zur Anwendung kommen immer 6 Kategorien: Patientenorientierung, Mitarbeiterorientierung, Sicherheit und Risikomanagement, Kommunikationsund Informationswesen, Unternehmensführung und schließlich Qualitätsmanagement. Sie werden durch Subkategorien und sog. „Kriterien“ weiter differenziert. Im derzeit aktuellen Katalog für Krankenhäuser (Katalog 2015) gibt es insgesamt 55 Kriterien. Ungefähr die Hälfte der Kriterien sind „Kernkriterien“, deren besondere Bedeutung für das Erreichen einer guten Qualität sich in der Anwendung eines Multiplikators auf die durch sie zu erreichende Punktzahl ausdrückt. Ein weiteres wesentliches Merkmal der Kataloge ist, dass sie nach dem PDCA-Zyklus aufgebaut sind. Die Bewertung der einzelnen Kriterien wird - in unterschiedlicher Gewichtung - auf die 4 Schritte des PDCA-Zyklus aufgeteilt. „Plan“ ist die Beschreibung des Soll-Zustandes mit Zielen und Kennzahlen, „Do“ ist die Beschreibung des Ist-Zustandes bzw. des Umsetzungsgrades, „Check“ ist die Beschreibung der Messverfahren und „Act“ die Beschreibung der Verbesserungsmaßnahmen. Für jeden einzelnen Schritt wird dann weiterhin die Hälfte der zu erreichenden Punkt für den sog. „Erreichungsgrad“ (Maß der Erfüllung der Anforderung) und die andere Hälfte für den sog. „Durchdringungsgrad“ (Maß der Umsetzung in der Breite) vergeben [28].

Der Zertifizierungsablauf erfolgt in 4 Schritten: Zunächst erfolgt eine Selbstbewertung, dann die Anmeldung bei einer der Zertifizierungsstellen, dann die Fremdbewertung durch KTQ-Visitoren und schließlich die Zertifizierung und Veröffentlichung des KTQQualitätsberichts. Das Zertifikat ist für 3 Jahre gültig.

Im Einzelnen gestaltet sich der Ablauf folgendermaßen [36]:

Ein an der KTQ-Zertifizierung interessiertes Krankenhaus führt zunächst eine Selbstbewertung anhand des KTQ-Katalogs unter Verwendung eines KTQ-Software-Tools durch. Weiterhin wird eine Kurzversion der Selbstbewertung, der sog. KTQ-Qualitätsbericht, erstellt, der zur späteren Veröffentlichung vorgesehen ist. Dazu ist es in der Regel erforderlich, einige Mitarbeiter auf KTQ-Kursen schulen zu lassen. Wenn in dieser Selbstbewertung ein erfolgversprechendes Ergebnis erreicht wird (bezogen auf die Mindestpunktzahl jeder Kategorie), kann das Krankenhaus für den nächsten Schritt einen Vertrag mit einer von der KTQ autorisierten Zertifizierungsstelle schließen und bei dieser Selbstbewertung und KTQ-Qualitätsbericht einreichen. Die Zertifizierungsstelle beauftragt ein Visitorenteam und stellt einen Visitationsbegleiter.

Das Visitorenteam besteht aus 3 Mitgliedern: einem ärztlichen Visitor (Chefarzt, stellv. Chefarzt oder Oberarzt), einem pflegerischen Visitor (Pflegedirektor oder stellv. Pflegedirektor) und einem ökonomischen Visitor (leitende Position in der Geschäftsführung eines Krankenhauses). Sie dürfen in ihrer jeweiligen Team-Zusammensetzung nicht aus dem gleichen Krankenhaus kommen. Die 
Visitorentätigkeit erfolgt strikt als Nebentätigkeit und ist in ihrer jährlichen Anzahl begrenzt. Dem Visitorenteam wird von der Zertifizierungsstelle ein Visitationsbegleiter zur Seite gestellt, der den gesamten Visitationsprozess organisiert und auf die Einhaltung der KTQ-Regeln achtet, bezüglich der Bewertung der Einrichtung durch die Visitoren jedoch Neutralität wahrt.

Dem Visitorenteam werden die Unterlagen des Krankenhauses übermittelt. Jeder Visitor prüft und bewertet die Unterlagen zunächst separat für sich. Die Visitoren besuchen anschließend gemeinsam das Krankenhaus, validieren die Selbstbewertung, sehen weitere Unterlagen ein und führen den sog. „kollegialen Dialog“, der dem Krankenhaus zusätzliche Impulse und Anregungen für sein Qualitätsmanagement geben soll. Die Visitation kann je nach Größe des Krankenhauses mehrere Tage dauern. Zum Abschluss fassen die Visitoren alle Informationen und Bewertungen im sog. KTQ-Visitationsbericht zusammen und geben eine Empfehlung (positiv oder negativ) bezüglich der Zertifikatsvergabe an die KTQ ab.

Die KTQ vergibt das Zertifikat an das Krankenhaus, wenn die Empfehlung der Visitoren positiv war, das Krankenhaus den KTQQualitätsbericht veröffentlicht und an der gesetzlich vorgeschriebenen externen Qualitätssicherung teilnimmt (nach DeQS-RL und/ oder QSKH-RL, s. 5.2.6.3 und 5.2.6.4). Für die Gültigkeitsdauer von 3 Jahren sind jährliche interne Audits vorgeschrieben, und eine ReZertifizierung nach Ablauf der Gültigkeit ist möglich und erwünscht. Für besonders herausragende Leistungen verleiht die KTQ jährlich den KTQ-Award. Auf der Website der KTQ sind anonymisierte Verteilungskurven der zertifizierten Einrichtungen für die Punkterreichung in den einzelnen Kategorien veröffentlicht, sodass jede Einrichtung die Möglichkeit zum Benchmark hat.

Die Zertifizierung von anderen Organisationen als Krankenhäusern verläuft im Prinzip gleich, ist aber im Katalog und der Zusammenstellung der Visitorenteams an die Spezifika der Organisationsart angepasst.

Für die Dauer der Gültigkeit des Zertifikats darf die Einrichtung mit ihm werben.

\subsubsection{KTQ-Plus}

Das KTQ-Plus-Verfahren ist eine freiwillige Ergänzung zur Zertifizierung, bei der durch „eine dezidierte Rückmeldung zu den abgestimmten Kriterien mit der Benennung von Stärken und Verbesserungspotenzialen als mündliches Feedback und in Form eines Berichts" die Entwicklung des einrichtungsinternen Qualitätsmanagements gefördert werden soll. Die Teilnahme an KTQ-Plus darf nach außen kommuniziert werden [37].

\subsubsection{KTQ Best Practice}

Mit der Best-Practice-Initiative will die KTQ die „Exzellenz im Qualitätsmanagement des Gesundheitswesens “ fördern und unternimmt damit einen Schritt über die reine Prozessorientierung hinaus. Alle KTQ-zertifizierten Einrichtungen sind aufgefordert, besonders gute Lösungen, innovative Projekte usw. zu präsentieren, um dadurch „verstärkt zum Austausch der Einrichtungen untereinander und zur Verbreitung hervorragender Lösungen“ beizutragen [38].

\subsubsection{Weiterentwicklung des Modells}

Das KTQ-Modell wird kontinuierlich aufgrund von Erfahrung und Nutzerfeedback, teilweise auch wegen geänderter gesetzlicher Vorgaben in der Qualitätssicherung weiterentwickelt.

So wurde im aktuellen Katalog 2015 für Krankenhäuser im Vergleich zur vorherigen Version 6.0 z. B. die zunehmende Bedeutung des Risikomanagements abgebildet und der Nachweis einer leitliniengerechten Behandlung als neue Anforderung eingebracht [39].

In den aktuellen Newslettern von April und Juli 2019 kündigt die KTQ weitreichende Veränderungen des Modells an:

- Der KTQ-Qualitätsbericht müsse von der Einrichtung nicht mehr erstellt werden, sondern ist zukünftig optional, weil über den gesetzlich verpflichtenden Qualitätsbericht (s. 5.2.6.1) schon eine ausreichende Information erfolge. Ziel sei die „Verfahrensvereinfachung und Aufwandsreduzierung“. Dasselbe Ziel verfolgen weiterhin Reduzierungen der Anforderungen für die Selbstbewertung.

- Es wird optional angeboten, im zweiten Gültigkeitsjahr des Zertifikats eine Zwischenbegehung durchzuführen, um Fortschritte bei gemeinsam vereinbarten Themen zu bewerten und eine zeitnahe Weiterentwicklung zu unterstützen. Die Dauer der Re-Zertifizierung nach 3 Jahren kann sich dadurch verkürzen.

- Der Bereich Risikomanagement und Patientensicherheit wird erneut in seiner Bedeutung erheblich aufgewertet werden.

- Das Feedback-Element des Zertifizierungsvorgangs soll deutlich gestärkt werden und das formative Element der Visitationsprüfung betont werden: Das Feedback der Visitoren soll Beratungscharakter haben. Sie sollen zukünftig zu jedem Kriterium der Selbstbewertung transparent ihre Einschätzung kommunizieren. Visitationsbegleiter sollen zukünftig, über ihrer neutrale Beobachterrolle hinaus, im Beratungsprozess mitwirken.

- Passend dazu soll die Bewertungsstruktur grundlegend überarbeitet werden. Ein prozentuales Gesamtergebnis, den dazugehörigen Benchmark und den KTQ-Award werde es zukünftig nicht mehr geben.

- Vielmehr gehe es „um die Förderung der Entwicklung der Qualität durch kollegiales Feedback“, es soll „der Fokus auf unsere Best-Practice-Initiative gesetzt“ werden und so der „Wissenstransfer aller KTQ-zertifizierten Einrichtungen“ gefördert werden.

Die Änderungen sollen zum 01.09.2019 in Kraft treten [40,41]. Die Betonung der kollegialen Beratung hat Analogien zum ärztlichen PeerReview-Verfahren der BÄK (s. 6.10.1). Hintergrund für die erkennbare, verstärkte Kundenorientierung könnte die abnehmende Gesamtzahl der zertifizierten Einrichtungen sein. Vor 10 Jahren waren fast 3-mal so viele Krankenhäuser zertifiziert wie derzeit [42].

\subsubsection{Pro cum cert (pCC) und pCC-KTQ-KH}

Pro cum cert ( $p C C$ ) ist nach eigener Aussage eine „werteorientierte Zertifizierungsgesellschaft“ für „Unternehmen und Einrichtungen des Gesundheits-, Sozial- und Bildungsbereichs, deren Ziele wertegebunden, gemeinnützig oder kirchlich geprägt sind“. Als Zertifizierungsgesellschaft bietet pCC ein großes Spektrum an Verfahren inkl. ISO 9001 und KTQ an. 
Zeitgleich mit der Entwicklung des KTQ-Modells wurde von pCC eine Erweiterung des KTQ-Katalogs für Krankenhäuser entwickelt, dass die Anforderungen an konfessionell getragene Häuser bezüglich ihrer „christlichen Werteorientierung “ berücksichtigt. Der pCCKTQ-KH-Katalog enthält neben den bekannten 6 Kategorien deshalb 3 zusätzliche Kategorien: Seelsorge im kirchlichen Krankenhaus, Verantwortung gegenüber der Gesellschaft und Trägerverantwortung. Die Zertifizierung nach pCC-KTQ-KH entspricht einer vollwertigen KTQ-Zertifizierung und gibt dem Krankenhaus darüber hinaus die Möglichkeit, sein christliches Profil nach innen und außen darzustellen [43].

\subsubsection{Relevanz}

KTQ hat im deutschen Gesundheitssystem noch eine relativ große Verbreitung. Nach eigenen Angaben sind aktuell 244 Krankenhäuser (Gesamtzahl Zertifikate: 2275), 41 Praxen und MVZ (Gesamtzahl Zertifikate: 220), 46 Rehabilitationseinrichtungen (Gesamtzahl Zertifikate: 275), 13 Pflegeeinrichtungen (Gesamtzahl Zertifikate: 104) und ein Rettungsdienst (Gesamtzahl Zertifikate: 12) zertifiziert [44]. Die Differenz aus der Zahl der aktuell zertifizierten Einrichtungen und der Gesamtzahl der Zertifikate ist bedingt durch die zeitliche beschränkte Gültigkeitsdauer der Zertifikate. Einrichtungen können mehrfach zertifiziert sein oder mittlerweile nicht mehr zertifiziert sein. Insgesamt ist die Zahl der zertifizierten Einrichtungen rückläufig (s. 4.3.5).

\subsection{EFQM - European Foundation for Quality Management}

\subsubsection{Geschichte und Organisation}

Ende der achtziger Jahre des letzten Jahrhunderts kamen europäische Politiker und Wirtschaftsführer zu der Ansicht, dass zum „Verbessern der Wettbewerbsfähigkeit der europäischen Unternehmen und zur nachhaltigen wirtschaftlichen Entwicklung Europas “(Übers. d. Verf. [45]) die alleinige Anwendung des bis dato gut etablierten ISO-Systems nicht mehr ausreicht, sondern ein europäisches TotalQuality-Management-System in Entsprechung des amerikanischen Malcom Baldrige National Quality Awards (1987) oder des japanischen Deming Prize (1951) entwickelt und ausgerollt werden sollte. Am 15. September 1988 trafen sich 14 leitende Manager aus großen europäischen Unternehmen mit Jaques Delors, dem damaligen Präsidenten der Europäischen Kommission, und unterzeichneten eine Absichtserklärung, eine europäische Stiftung mit dem Ziel der Verbesserung der europäischen Wettbewerbsfähigkeit zu gründen. Ein Jahr später wurde die European Foundation for Quality Management unter Beteiligung von 67 europäischen Unternehmen gegründet. Ein Entwicklungsteam wurde eingerichtet, um ein holistisches, auf alle Formen von Organisationen anwendbares Modell zu entwickeln, das mit der Vergabe des ersten European Quality Award (EQA) 1992 in Madrid erstmals zur Anwendung kam. Das EFQM-Modell wurde bei den Revisionen 1999, 2003, 2010 und 2013 teils deutlich, teils behutsam überarbeitet, um es fortzuentwickeln und an die Bedürfnisse der Organisationen anzupassen. Für das Jahr 2020 steht der nächste Versionssprung an (s. 4.4.3). Der EQA wurde im Jahr 2006 in den European Excellence Award (EEA) umbenannt. Auf nationaler Ebene wird er durch den erstmals im Jahr 1997 vergebenen Ludwig Erhard Preis (ILEP [46]) ergänzt. Das EFQM-Modell wurde im Jahr 1996 um die Kategorien „öffentlicher Sektor“ und 1997 „kleine und mittelständige Unternehmen“ durch entsprechende Awards erweitert. Als vierte Gruppe können sich auch Organisationseinheiten um eine Anerkennung bewerben. Im Jahr 2001 wurde eine „EFQM Knowledge Base “ in Betrieb genommen und ein abgestuftes Zertifikatsystem („Levels of Excellence“) zur Ergänzung des jährlichen Hauptpreises eingeführt [47].

Die EFQM gibt an, derzeit 450 Mitglieder (Firmen und öffentliche Einrichtungen), 48 Partnerorganisationen (nationale Qualitätsorganisationen), 1500 Assessoren und 50000 Anwender zu haben.

\subsubsection{Verfahren}

Der Exzellenz-Gedanke ist das zentrale Motiv des EFQM-Modells, das sich als Instrument und Grundstruktur für das Managementsystem einer erfolgreichen Organisation versteht. Es soll eine ganzheitliche Bewertung und Steuerung einer Organisation ermöglichen, indem es ihre relevanten Elemente auf ihre Funktion im Kontext mit anderen Elementen und ihr Zusammenwirken mit ihnen untersucht. Der Fokus ist dabei immer auf eine langfristige, nachhaltige Exzellenz gerichtet. Das EFQM Exzellenz-Modell besteht aus 3 ineinandergreifenden Komponenten: den „Grundkonzepten der Exzellenz“, dem „Kriterien-Modell“ und der „RADAR-Logik“.

\subsubsection{Die acht Grundkonzepte}

Die 8 Grundkonzepte der Exzellenz beschreiben die prinzipiellen Voraussetzungen dafür, als Organisation Exzellenz zu erreichen, und stellen wichtige Leitlinien für die Organisationsführung dar. Im Einzelnen werden genannt:

- Nutzen für Kunden schaffen

- Die Zukunft nachhaltig gestalten

- Die Fähigkeiten der Organisation entwickeln

- Kreativität und Innovation fördern

- Mit Vision, Inspiration und Integrität führen

- Veränderungen aktiv managen

- Durch Mitarbeiterinnen und Mitarbeiter erfolgreich sein

- Dauerhaft herausragende Ergebnisse erzielen [48].

Auf diesen Kanon an Grundkonzepten bauen die beiden Bewertungselemente, d. h. das Kriterien-Modell und die RADAR-Logik, auf. Die Bewertung erfolgt prinzipiell immer zunächst als Selbstbewertung und kann bei Bewerbung um eine Auszeichnung durch eine Fremdbewertung ergänzt werden.

\subsubsection{Das Kriterienmodell}

Das Ziel des Kriterienmodells ist laut EFQM, es den Führungskräften zu erlauben, „die Ursache-Wirkungs-Zusammenhänge zwischen dem, was die Organisation tut und den resultierenden $\mathrm{Er}$ gebnissen besser zu verstehen“.

Das Kriterienmodell ist die übergeordnete Bewertungsstruktur. Sie enthält 9 Kriterien, die sich auf die Aktivitäten und die Ergebnisse einer Organisation beziehen. Die 5 aktivitätsbezogenen Kriterien werden „Befähigerkriterien“ genannt, die 4 ergebnisbezogenen „Ergebniskriterien“. Den 9 Kriterien sind insgesamt 32 Teilkriterien zugeordnet und jedem Teilkriterium mehrere erläuternde sog. „Orientierungspunkte“, die den Bezug zu den acht Grundkonzepten herstellen.

Die 5 Befähigerkriterien lauten: 
1. Führung

2. Strategie

3. Mitarbeiter

4. Partnerschaften und Ressourcen

5. Prozesse, Produkte und Dienstleistungen

Dem Kriterium Strategie sind 4 Unterkriterien zugeordnet, den anderen jeweils 5 .

Die 4 Ergebniskriterien lauten:

6. Kundenbezogene Ergebnisse,

7. Mitarbeiterbezogene Ergebnisse,

8. Gesellschaftsbezogene Ergebnisse

9. Schlüsselergebnisse

Ihnen sind jeweils 2 Teilkriterien zugeordnet.

Der Aufbau dieses Modells erlaubt es, die Organisation als Ganzes aus der PDCA-Perspektive zu betrachten: Die Befähigerkriterien entsprächen dem „Plan“ und „Do“, die Ergebniskriterien dem „Check“ und die Feedback-Schleife zu den Befähigern, die von der EFQM als „Lernen, Kreativität, Innovation“ bezeichnet wird, entspräche dem „Act“.

Insgesamt werden bei der Bewertung der 9 Kriterien maximal 1000 Punkte vergeben, die weitgehend gleichmäßig auf die Kriterien verteilt sind. Die 2 ergebnisbezogenen Kriterien „6. Kundenbezogene Ergebnisse“ und „9. Schlüsselergebnisse“ werden mit je $15 \%$ bzw. 150 Punkten stärker gewichtet, alle anderen Kriterien werden mit $10 \%$ bzw. 100 maximal erreichbaren Punkten gewichtet. Dadurch ist die Gesamtpunktzahl hälftig auf Befähiger- und Ergebniskriterien verteilt.

\subsubsection{Die RADAR-Logik}

Die Methode, die einzelnen Teilkriterien zu bewerten und den jeweiligen Prozentsatz der erreichten Punkte zu vergeben, wird in der EFQM-Terminologie „RADAR-Logik“ genannt. RADAR steht für Results, Approach, Deployment sowie Assessment und Refinement, ist für die Bewertung von Befähiger- und Ergebniskriterien spezifisch angepasst und insgesamt vom PDCA-Zyklus-Prinzip abgeleitet (mit der Ergänzung des „Ergebnisses“). Die dahinterstehende Überlegung ist, dass zunächst die Zieldefinition einer Organisation über die gewünschten Ergebnisse erfolgt („R“), dann die passenden Herangehensweisen entwickelt werden („A“ = „Plan“), diese umgesetzt ( „D“ = „Do“) und anschließend evaluiert und ggf. optimiert werden („AR“ = „Check \& Act“).

Für die Bewertung werden die „RADAR-Elemente“ auf die Befähiger- und Ergebniskriteriengruppen aufgeteilt:

Bei den Befähiger-Teilkriterien werden Vorgehen (Approach), Umsetzung (Deployment) und Bewertung und Verbesserung (Assessment and Refinement) angewendet. Für die Ergebnis-Teilkriterien wird das Ergebniselement (Results) in „Relevanz und Nutzen“ und „Leistung“ aufgeteilt. Allen RADAR-Elementen werden individuell 2-4 „Attribute“ wie z. B. „Fundiert“, „Eingeführt“, „Messung“, „Umfang“, „Trends“ usw. zugeordnet.

Auf der Ebene der Attribute findet dann die eigentliche Bewertung anhand einer Matrix statt, die eine fünffache Segmentierung aufweist von „keine Nachweise“ (= 0 \%) über „einzelne Nachweise“ (= $25 \%$ ), „Nachweise“ ( = $50 \%$ ), „klare Nachweise“ (= $75 \%$ ) bis „durchgängig vorbildlich“ (=100\%).

\subsubsection{Ablauf des Verfahrens, Bewerbung um Anerkennung} und Preise

Im Vordergrund steht im EFQM-Modell der Prozess der Selbstbewertung. Mit der notwendigen Kompetenz für das Modell durch Ausbildung eigener Mitarbeiter und ggf. mithilfe externer Berater kann die Selbstbewertung Verbesserungspotenziale in allen Aspekten des eigenen Managementsystems aufzeigen. Wenn diese durch entsprechende Maßnahmen genutzt werden, kann in einem iterativen Prozess die Qualität der Gesamtorganisation gesteigert werden.

Wenn der Wunsch besteht, das erreichte eigene Qualitätsniveau einer externen Beurteilung zu unterziehen und es nach außen zu kommunizieren, kann an dem 4-stufigen „EFQM Recognition“-Programm teilgenommen werden [49]. Das je nach Stufe und damit verbundenem Aufwand unterschiedlich ausführliche Feedback der externen Gutachter (sog. „Assessoren“) kann als Ausgangspunkt weiterer Verbesserungsinitiativen dienen:

1. Committed to Excellence Validation

Beim Einstiegslevel werden nach der Selbstbewertung und dem damit verbundenen Identifizieren von Verbesserungspotenzialen 3 Verbesserungsprojekte durchgeführt. Ein EFQM-Validator wird während eines Ein-Tages-Besuchs die Ergebnisse dieser 3 Projekte beurteilen und einen diesbezüglichen Feedback-Bericht erstellen. Wenn ein klarer Fortschritt festgestellt werden kann, wird die Anerkennung „Committed to Excellence 1 Star" verliehen.

2. Committed to Excellence Assessment Beim nächsten Level werden die wichtigsten Elemente des Managements untersucht: Strategie und Schlüsselergebnisse, das Management von Mitarbeitern, Kunden und Prozessen und die Nachhaltigkeit. Zusätzlich zur Selbstbewertung ist eine kurze Selbstbeschreibung zu erstellen. Zwei EFQM-Assessoren beurteilen während eines Ein-Tages-Besuchs die Organisation unter o.g. Aspekten. Anschließend wird ein mündliches Feedback gegeben sowie ein ausführlicher schriftlicher Bericht. Wenn in der Bewertung mehr als 200 Punkte erreicht werden, wird die Anerkennung „Committed to Excellence 2 Star“ verliehen.

3. Recognised for Excellence

Fortgeschrittene Organisationen können sich einer vollständigen externen Beurteilung durch 3-5 Assessoren in einem Zeitraum von 3-5 Tagen unterziehen. Anschließend erfolgt ein umfassendes schriftliches Feedback sowie die Verleihung des Zertifikats „Recognized for Excellence 3 Star, 4 Star or 5 Star“. Die Vergabe der Sterne hängt davon ab, ob die Organisation einige (mindestens 300 Punkte), gute (mindestens 400 Punkte) oder sehr gute (mindestens 500 Punkte) ChangeManagement-Aktivitäten und Ergebnisse zeigt.

4. EFQM Global Excellence Award Organisationen, die mit „Recognized for Excellence 5 Star” bewertet wurden, können sich für den jährlichen Global Excellence Award bewerben. Nach einem Vortreffen erfolgt eine Beurteilung durch 5-9 Assessoren an 5 Tagen. Anschließend entscheidet eine EFQM-Jury über die jährliche Preisvergabe. Es kann eine Einstufung als „Highly Commended“, „Prize Winner“ in einem oder mehreren fundamentalen Qualitätskonzepten oder als „Award Winner“ erfolgen. Weiterhin erfolgt ein extensives schriftliches Feedback. 


\section{Zusätzlich können sich deutsche Organisationen um den} nationalen Ludwig-Erhard-Preis bewerben [46].

\subsubsection{Weiterentwicklung des Modells}

Auch für das EFQM-Modell ist für den Herbst 2019 eine sehr weitgehende Umgestaltung in der kommenden Modell-Version 2020 angekündigt. Anstatt wie bisher den Fokus nur auf die kontinuierliche Verbesserung zu legen, die in den letzten 25 Jahre im Mittelpunkt gestanden habe, gebe es einen Bedarf in Organisationen, mit Transformationen und disruptiven Ereignissen durch effektives ChangeManagement genauso gut umgehen zu können wie mit kontinuierlicher Verbesserung. Das EFQM-Modell 2020 werde sich daran stark anpassen und eher ein Management-Werkzeug anstelle des bisherigen Assessment-Werkzeugs werden. Weiterhin werde das gesamte EFQM-Verfahren vollständig digitalisiert werden. Es werde für Mitglieder eine kuratierte Version der Wissens-Plattform („Knowledge Base“) eingerichtet sowie eine Assessment-Plattform („Assessment Base“). Letztere werde es Organisationen erlaube, eine Selbstbewertung durchzuführen und zu testen, ob das EFQM-Modell für sie praktikabel sei. Zusätzlich werde ein eigenes, exklusives und App-basiertes soziales Netzwerk namens „Totem“ eigerichtet, in dem sich Mitglieder nicht nur darstellen können, sondern das als Best-PracticePlattform dienen solle und über das unkomplizierter Zugang zur Knowledge Base und zur Assessment Base ermöglicht werden solle [50-52].

\subsubsection{Relevanz}

Im Vergleich zu den Branchen von Wirtschaft und Verwaltung ist die Anwendung des EFQM-Modells bei Organisationen im Gesundheitswesen, die zu den Leistungserbringern gerechnet werden können, relativ überschaubar- der „Healthcare Sector“, der auch Verwaltungsorganisationen, Pharmaindustrie usw. einschließt, umfasst nur $6 \%$ aller EFQM-Anwender. Für die einem Zertifikat entsprechenden „Recognitions“ der unterschiedlichen Stufen werden für Deutschland derzeit 14 Kliniken, davon eine Uni-Klinik, 3 Organisationseinheiten von Kliniken, 2 Praxen und 1 MVZ genannt [53].

\subsection{Vergleich ISO, KTQ, EFQM}

Von den 3 Modellen ist ISO am stärksten prozessorientiert und EFQM als Exzellenzmodell am stärksten ergebnisorientiert. KTQ bewegt sich zwischen den beiden. Alle 3 Systeme beginnen mit einer Selbstbewertung und bieten anschließend eine externe Evaluation durch Auditoren, Visitoren oder Assessoren. Nur ISO und KTQ erteilen ein Zertifikat und bewerten damit den Ist-Zustand, während bei EFQM die Ausrichtung auf die Zukunft und das Zusammenspiel der Unternehmensbereiche im Fokus steht. Der entscheidende Unterschied ist, dass Qualitätsindikatoren und Konformitätsbescheinigungen retrospektiv sind, während Exzellenzmodelle eine vorausschauende Steuerung der Organisation erlauben.

Alle Verfahren legen Wert auf einen kontinuierlichen Verbesserungsprozess. KTQ ist das einzige Gesundheitssystem-spezifische Verfahren, ISO und EFQM sind dagegen generisch [54]. Von Anwendern und Beratern wird ISO als niedrigschwelliges System angesehen, das einen guten Einstieg in das Einrichten eines Qualitätsmanagementsystems bietet. KTQ hat den Vorteil, die Prozesse im Krankenhaus oder der Praxis bereits explizit abzubilden, ist aber in der
Umsetzung aufwendiger. EFQM ist das abstrakteste Modell von allen, Vorerfahrung mit Qualitätsmanagementsystemen wird empfohlen [28].

\subsection{Zertifizierung von Praxen und MVZ - QEP, ISO, KPQM, KTQ, EPA u. a}

Vertragsärztliche Praxen und MVZs unterliegen genauso wie Krankenhäuser der gesetzlichen Verpflichtung, ein einrichtungsinternes Qualitätsmanagement zu betreiben (§135a SGB V). Die vom Gemeinsamen Bundesausschuss (G-BA) dazu herausgegebene Qualitätsmanagementrichtlinie (QM-RL) legt dazu die Einzelheiten fest (s. 5.2.5.1).

Eine freiwillige Zertifizierung kann bei der Verbesserung und kontinuierlichen Weiterentwicklung des Qualitätsmanagementsystems sehr hilfreich sein. Neben den generischen, oben bereits beschriebenen Systemen nach ISO, KTQ und EFQM gibt es auch praxis- bzw. MVZ-spezifische Zertifizierungssysteme. Am weitesten verbreitet ist QEP (Qualität und Entwicklung in Praxen, Anbieter Kassenärztlichen Bundesvereinigung (KBV) [55, 56]), mit deutlichem Abstand gefolgt vom KPQM (KV-Praxis-Qualitätsmanagement, Anbieter Kassenärztliche Vereinigung Westfalen-Lippe [57]) und dem EPA Praxissiegel (Europäisches Praxisassessment, Anbieter Aqua-Institut [58]). Darüber hinaus gibt es noch eine Anzahl weiterer Anbieter mit kleinerem Marktanteil. Die KBV gibt die Anteile der Anwender der oben genannten Verfahren anhand der jährlichen Stichprobe (s. 4.6.2) von 2017 mit QEP 32\%, DIN EN ISO 9001:2015 26\%, KPQM 4\%, KTQ PRAX-MVZ3.0 2\% und EPA $2 \%$ an. EFQM spielt bei derzeit deutschlandweit 3 Teilnehmern aus diesem Segment keine Rolle (s. 4.4.4).

\subsubsection{Verfahren}

Als einziges Verfahren ist ISO rein generisch, KTQ adaptiert sein Verfahren durch einen speziell abgestimmten Katalog. Alle anderen 3 Verfahren wurden speziell für den vertragsärztlichen Bereich entwickelt.

Allen Verfahren ist die prinzipielle Vorgehensweise gemein, ein kriterienbasiertes Selbst- und Fremdbewertungssystem zu sein. Nach Vorbereitung und Selbstbewertung findet die externe Bewertung durch einen Auditor bzw. Visitor statt, und im Erfolgsfall wird bei allen Systemen ein für 3 Jahre gültiges Zertifikat verliehen. Bei QEP, ISO und KTQ wird erwartet, dass bei einer Re-Zertifizierung die Leistungen messbar verbessert werden können. Die Kosten für Schulungen und Zertifizierungen liegen in vergleichbaren Größenordnungen $[59,60]$.

\subsubsection{Relevanz}

Der jährlich von der KBV abzugebende „Bericht zum Umsetzungsstand von Qualitätsmanagement in Vertragsarztpraxen und MVZ gemäß QM-RL Teil A §7“ wurde den durch Stichprobe ausgewählten Teilnehmern die Frage nach „Einsatz spezifischer QM-Systeme“ gestellt, deren Beantwortung freiwillig war. $54 \%$ der Befragten gaben daraufhin an, diese Systeme einzusetzen (Verteilung siehe 4.6), und $13 \%$ gaben an, sich schon einmal einer freiwilligen Zertifizierung unterzogen zu haben (s. 5.2.6.2), d. h. dreiviertel der Anwender belassen es bei der Selbstbewertung. 


\subsection{Zertifizierung von Zentren}

\subsubsection{Geschichte und Organisation}

Das Ziel einer Zentrenbildung ist im Regelfall, einen Mehrwert gegenüber der konventionellen Versorgung zu schaffen. Ein Zentrum kann beschrieben werden als eine „Aggregation von Expertise und Ressourcen" [61].

Prinzipiell kann man Zentrenbildungen unterscheiden in solche, die durch Bündeln von Ressourcen (z.B. Endoskopiezentren) entstehen, von Gesundheitsorganisationen ausgehen und meistens nicht zertifiziert sind, und in Zentrumszertifizierungen für bestimmte Krankheitsbilder durch Fachgesellschaften. Darüber hinaus gibt es staatliche Zentrenausweisungen z. B. durch Krankenhauspläne, Tumorzentren und onkologische Schwerpunkte in Baden-Württemberg, Brustzentren in Nordrhein-Westfalen oder auch für MVZs.

Die Zentrenzertifizierungen durch Fachgesellschaften, die sich auf krankheitsorientierte Zentren beziehen, lassen sich in Zentren in der Tumorversorgung und Zentren in anderen Fachgebieten unterteilen.

Zentren in der Tumorversorgung werden nach „Onkozert“, dem Zertifikationsverfahren der Deutschen Krebsgesellschaft (DKG) und der Deutschen Krebshilfe (DKH), zertifiziert, wie z. B. für unser Fachgebiet die Kopf-Hals-Tumorzentren [62]. Je nach Größe und Bedeutung werden sie als „Organkrebszentrum (C)“, „Onkologisches Zentrum (CC)“ oder „Onkologisches Spitzenzentrum (CCC)“ klassifiziert. Darüber hinaus existieren weitere, fachgesellschaftsgebundene onkologische Zertifizierungsverfahren. Die Zentren für bedeutende nicht-onkologische Krankheitsbilder werden von den zuständigen Fachgesellschaften oder Fachorganisationen zertifiziert.

\subsubsection{Verfahren}

Ein krankheitsorientiertes Zentrum sollte der Bundesärztekammer entsprechend folgende Eigenschaften aufweisen:

- Krankheitsorientierte Spezialisierung

- Patientenzentrierung

- Evidenzbasierte Versorgung

- Interdisziplinäre und -professionelle Kooperation und Kommunikation

- Enge Kooperation und Kommunikation mit den einweisenden und weiterbetreuenden Ärzten sowie mit allen anderen an der Versorgung des Patienten Beteiligten

- Qualitätsmanagement [63]

Die eigentliche Zertifizierung erfolgt im Regelfall nach den Prinzipien einer ISO-Zertifizierung mit Selbstbewertung anhand eines Anforderungskatalogs, Fremdbewertung durch ein Audit und Zertifikatserteilung mit begrenzter Laufzeit.

\subsubsection{Relevanz}

Auf dem Gebiet der Zertifizierung als Teil eines Zentrums gibt es im Unterschied zu den vorgenannten Verfahren eine Dynamik des Peer-Pressure, d. h. es wird die Notwendigkeit gesehen, sich zu beteiligen, sich „auch“ zu zertifizieren, um nicht ein negatives Distinktionsmerkmal aufzuweisen.

Die Bundesärztekammer und der 112. Deutsche Ärztetag haben die teilweise inflationäre Ausbreitung der Zentrumsgründung in der deutschen Krankenhauslandschaft kritisiert [64]. In der allgemeinen Wahrnehmung sei der Zentrumsbegriff positiv mit Kom- petenz, Spezialisierung und umfassender Behandlung verknüpft. Da er nicht geschützt sei, könne sich letztlich jede Einrichtung als Zentrum bezeichnen, um z. B. den damit verbundenen Marketingeffekt zu nutzen. Zentren müssen nicht zwangsläufig zertifiziert sein, führen aber oft eine Zertifizierung an. Das „Institut für Qualität und Transparenz im Gesundheitswesen“ (IQTIG) wurde vom Gesetzgeber beauftragt, in diesem Kontext „Kriterien zur Bewertung von Zertifikaten und Qualitätssiegeln im ambulanten wie stationären Bereich“ zu entwickeln (§135a Absatz 3 SGB V, s. 5.3.2). Die Bundesärztekammer schließt sich bei der Bewertung von Zertifizierungen inhaltlich einem Grundsatzpapier der Schweizer Akademie der Wissenschaften an, in dem an einen medizinischen Zertifizierungsvorgang Anforderungen analog zu einer ISO-Zertifizierung gestellt werden [65].

\subsection{Diskussion - Messbarer Nutzen?}

Die naheliegende Frage, ob eine externe Zertifizierung einen messbaren Nutzen für die patienten- und organisationsbezogenen Prozesse und Ergebnisse bewirkt, ist erstaunlicherweise nicht eindeutig zu beantworten, weil es keine systematischen, aussagekräftigen Untersuchungen dazu gibt, wie in 2 Cochrane Reviews festgestellt wurde $[66,67]$.

Ein Vergleich von US-Krankenhäusern, die von der Joint Commission akkreditiert waren, mit staatlich akkreditierten ergab keinen Unterschied bei Mortalität und 30-Tage-Wiederaufnahmequote [68]. Eine Erhebung der Patientenzufriedenheit nach stationärer Behandlung an deutschen Krankenhäusern ergab keinen Unterschied zwischen KTQ-, pCC- und ISO-zertifizierten Krankenhäusern und nicht-zertifizierten [69]. Auch andere, ähnliche Untersuchungen ergaben bestenfalls gemischte Resultate. Allerdings genügen die hier verwendeten Qualitätsindikatoren bezüglich ihrer Aussagekraft nicht höheren Ansprüchen.

Die AG Zentren der Bundesärztekammer konnte bei ebenfalls schlechter Studienlage für krankheitsorientierten Zentrenbildung nur fraglich messbare Effekte auf die Versorgung feststellen [70].

Von Seiten der Joint Commission wird darauf hingewiesen, dass es schwierig sei, mit Ausnahme der Mortalität reliable Qualitätsindikatoren zur Ergebnisqualität zu definieren. Die Herausforderung, valide Qualitätsindikatoren zu entwickeln, ist offenbar erheblich und besteht auch im System der gesetzlichen externen Qualitätssicherung in Deutschland (s. 5.12).

Innovative Ansätze mit geringem Aufwand können valide Aussagen über Qualitätsmängel von Krankenhäusern ermöglichen, wie in Großbritannien die Kombination aus klassischer Befragung von Patienten und Auswertung ihrer Aussagen auf Social-Media-Kanälen ergab [71].

Bezüglich der Wirkung innerhalb der Organisationen gibt es etliche Vorher-nachher-Untersuchungen, die Mitarbeiterzufriedenheit, interprofessionelle Zusammenarbeit, Effizienz der Organisation usw. nach Zertifizierung bzw. Akkreditierung signifikant verbessert sehen $[72,73]$.

Es bleibt festzustellen, dass eine Zertifizierung bzw. Akkreditierung per se keine Aussage über die Ergebnisqualität machen kann, sondern nur über die Voraussetzungen dafür, gute Qualität zu erreichen. Aus diesem Grund beinhalten alle relevanten Systeme heute zusätzlich die Abfrage von Qualitätsindikatoren und die Not- 
wendigkeit, bei Wiederholungszertifizierungen positive Entwicklungen zu demonstrieren [65].

Die Tatsache, dass von kommerziellen Unternehmen Zertifizierungen, Akkreditierungen und insbesondere Exzellenzmodelle trotz des damit verbundenen, nicht unerheblichen Aufwands eingesetzt werden, zeigt, dass offenbar die Einschätzung besteht, das mit ihrem Einsatz ein echter Mehrgewinn verbunden ist.

Trotzdem ist es zutiefst unbefriedigend, dass es keine solide Evidenz für die Effekte einer externen Zertifizierung oder Akkreditierung auf die medizinische Ergebnisqualität gibt. Methodisch gute, randomisierte kontrollierte Studien (RCT) wären äußerst wünschenswert, diese Frage zu beantworten (s. 8.4).

\section{Gesetzliche und institutionelle Qualitäts- sicherung (verpflichtende externe Qualitäts- sicherung)}

Das Gesundheitswesen in Deutschland ist nicht durchgehend staatlich organisiert, sondern als sog. gemeinsame Selbstverwaltung, wobei die Hauptakteure als Körperschaften des öffentlichen Rechtes verfasst sind. Auf der Leistungserbringerseite sind das die Repräsentanten der Ärzte und Zahnärzte und des stationären Versorgungssektors, und auf der Kostenträgerseite sind das v. a. die gesetzlichen Krankenversicherungen. Praktisch alle Institutionen der gemeinsamen Selbstverwaltung im Gesundheitswesen sind auch mit Qualitätsfragen oder Aspekten davon befasst. Von daher ist eine Darstellung des institutionellen Qualitätsmanagements gleichzeitig auch eine nahezu lückenlose Darstellung der Strukturen der gemeinsamen Selbstverwaltung des Gesundheitswesens, ihrer Unterorganisationen und Spin-offs.

\subsection{Qualitätsrelevante Gesetze}

Der Großteil der gesetzlichen Regelungen, die die Frage der Qualität im Gesundheitswesen betreffen, finden sich im fünften Buch des Sozialgesetzbuchs (SGB V), das 1989 als Ablösung des zweiten Buchs der Reichsversicherungsordnung in Kraft trat.

Drei sehr relevante Paragrafen definieren die Leistungsansprüche der gesetzlich Versicherten in Deutschland:

Erstens das „Wirtschaftlichkeitsgebot“ $§ 12$ SGB V:

„(1) Die Leistungen müssen ausreichend, zweckmäßig und wirtschaftlich sein; sie dürfen das Maß des Notwendigen nicht überschreiten. Leistungen, die nicht notwendig oder unwirtschaftlich sind, können Versicherte nicht beanspruchen, dürfen die Leistungserbringer nicht bewirken und die Krankenkassen nicht bewilligen".

Zweitens wird im Paragrafen „Leistungen“ § 2 SGB V u. a. das Niveau der unter Berücksichtigung des Wirtschaftlichkeitsgebots zu erbringenden Leistungen definiert:

„(1).... Qualität und Wirksamkeit der Leistungen haben dem allgemein anerkannten Stand der medizinischen Erkenntnisse zu entsprechen und den medizinischen Fortschritt zu berücksichtigen“.
Drittens wird im Paragrafen „Qualität, Humanität und Wirtschaftlichkeit“ § 70 SGB V zusammenfassend auf das Zusammenspiel von Versorgungsgerechtigkeit, Wirtschaftlichkeit und Ethik hingewiesen:

(1) Die Krankenkassen und die Leistungserbringer haben eine bedarfsgerechte und gleichmäßige, dem allgemein anerkannten Stand der medizinischen Erkenntnisse entsprechende Versorgung der Versicherten zu gewährleisten. Die Versorgung der Versicherten muss ausreichend und zweckmäßig sein, darf das Maß des Notwendigen nicht überschreiten und muss in der fachlich gebotenen Qualität sowie wirtschaftlich erbracht werden.

(2) Die Krankenkassen und die Leistungserbringer haben durch geeignete Maßnahmen auf eine humane Krankenbehandlung ihrer Versicherten hinzuwirken (Unterstreichungen d. d. Verf.).

Das SGB V regelt weiterhin in den $\S \S 95 \mathrm{~d}$ und 136b die Fortbildungspflicht für Fachärzte.

\subsubsection{GKV-Modernisierungsgesetz 2004}

Die meisten qualitätsspezifischen gesetzlichen Regelungen wurden mit Beginn des neuen Jahrtausends eingeführt, insbesondere mit dem Inkrafttreten des Gesundheitsmodernisierungsgesetzes (GKV-Modernisierungsgesetz) [74] im Jahr 2004, das eine umfassende Reform und Erweiterung des SGB V beinhaltete. Die wichtigsten Regelungen betreffen

- die Verpflichtung der Leistungserbringer zum Einrichten eines internen Qualitätsmanagements und zur Teilnahme an externen Qualitätssicherungsmaßnahmen (§ 135a SGB V) und

- das Einrichten des Gemeinsamen Bundesauschusses (G-BA, $\S 91$ SGB V, s. 5.2), das Festlegen seiner Kompetenzen (§§ 136 und 137 SGB V) sowie das Gründen des ihm zuarbeitenden Instituts für Qualität und Wirtschaftlichkeit im Gesundheitswesen (IQWIG, §139a SGB V, s. 5.4).

\subsubsection{G-DRG}

Auch innerhalb des im Jahr 2004 verpflichtend eingeführten G-DRG-Systems ( $\$ 85$ SGB V und § 17b Krankenhausfinanzierungsgesetz) wurden z. B. mit der „unteren Grenzverweildauer“ Regelungen zum Sichern von Qualitätsmindeststandards eingeführt.

\subsubsection{GKV-Wettbewerbsstärkungsgesetz (GKV-WSG)}

Mit dem GKV-Wettbewerbsstärkungsgesetz wurde 2007 u. a. die Kosten-Nutzen-Bewertung (KNB) von Arzneimitteln nach §35 SGB $\checkmark$ eingeführt.

\subsubsection{Arzneimittelmarktneuordnungsgesetz (AMNOG)}

Das Arzneimittelmarktneuordnungsgesetz führte 2011 u. a. das Verfahren der frühen Nutzenbewertung von Arzneimitteln in Verantwortung des G-BA ein (§35a Absatz 1 SGB V [75]).

\subsubsection{GKV-Versorgungsstrukturgesetz (GKV-VStG)}

Mit dem Versorgungsstrukturgesetz wurde 2012 u. a. die Erprobungsregelung für Untersuchungs- und Behandlungsmethoden eingeführt. Sowohl der G-BA als auch die Industrie können dazu Studien initiieren. 


\subsubsection{Patientenrechtegesetz}

Das im Jahr 2013 in Kraft getretenen Gesetz regelt zusammenfassend die Rechte von Patienten

- gegenüber den Behandlern in Bezug auf Informations- und Aufklärungspflichten, Dokumentation der Behandlung und Einsichtsrechte der Patienten,

- gegenüber den Kostenträgern,

- in Bezug auf die Beteiligung in Gremien der Selbstverwaltung und

- in Bezug auf ein Risiko- und Fehlermanagement bei den Behandlern [76].

5.1.7 GKV-Finanzstruktur- und Qualitätsweiterentwicklungsgesetz (GKV-FQWG)

Im GKV-Finanzstruktur- und Qualitätsweiterentwicklungsgesetz von 2015 wurde u. a. die Gründung des Instituts für Qualität und Transparenz im Gesundheitswesen (IQTIG) beschlossen (s. 5.3).

\subsubsection{GKV-Versorgungsstärkungsgesetz (GKV-VSG)}

Mit dem GKV-Versorgungsstärkungsgesetz wurde 2015 u. a. das Entlassmanagement ( $§ 39$ Absatz 1a SGB V) und ein Procedere für neue Untersuchungs- und Behandlungsmethoden (NUB, §137h SGB V) eingeführt.

\subsubsection{Krankenhausstrukturgesetz (KHSG)}

Mit dem Krankenhausstrukturgesetz wurden 2016 Qualitätsmaßstäbe erstmals als Kriterium für die Krankenhausplanung eingeführt. Der G-BA sollte dazu relevante Qualitätsindikatoren entwickeln und seine Ergebnisse den Beteiligten an der Krankenhausplanung zukommen lassen. Den Ländern wurde das Recht eingeräumt, das Berücksichtigen der Ergebnisse im Krankenhausplan auszuschließen (s. 5.2.6.8).

\subsubsection{Pflegepersonal-Stärkungsgesetz (PpSG)}

Im Pflegepersonal-Stärkungsgesetz wurde 2019 die Finanzierung der Pflegepersonalkosten aus dem DRG-System ausgegliedert und die Vollfinanzierung von Tarifsteigerungen festgelegt. Es wurden weiterhin Pflegepersonaluntergrenzen in pflegesensitiven Bereichen festgelegt ( $§ 137 i$ SGB V). Zum Sichern der Versorgung durch ländliche Krankenhäuser wurde ein Sicherstellungszuschlag eingeführt [77].

\subsubsection{Terminservice- und Versorgungsgesetz (TSVG)}

Mit dem Terminservice- und Versorgungsgesetz wurde 2019 die Mindestsprechstundenzahl für Vertragsärzte auf 25 pro Woche erhöht. Bestimmte Facharztgruppen, u.a. HNO-Ärzte, sind verpflichtet, 5 offene Sprechstunden pro Woche anzubieten. Die Terminservicestellen (TSS), zukünftig bundeseinheitlich unter der Tel. -Nr. 116117 zu erreichen, sollen weiter ausgebaut werden und auch die Steuerung von Notfallpatienten übernehmen (s. auch 8.1.4). Über die TSS vermittelte sowie Behandlungen in offenen Sprechstunden sollen extrabudgetär vergütet werden.

\subsubsection{Weitere qualitätsrelevante Gesetze}

Weitere qualitätsrelevante Gesetze betreffen Fragen der Patientensicherheit (Infektionsschutzgesetz und Empfehlungen der „Kommission für Krankenhaushygiene und Infektionsprävention“ (KRINKO) beim Robert Koch-Institut, Arzneimittelgesetz, Medizinpro- duktegesetz, Medizinproduktebetreiberverordnung u. a.) und des Mitarbeiterschutzes (Arbeitszeitgesetz).

\subsection{G-BA - Gemeinsamer Bundesausschuss}

Der Gemeinsame Bundesausschuss (G-BA) [78] ist nach eigener Aussage „das höchste Beschlussgremium der gemeinsamen Selbstverwaltung im deutschen Gesundheitswesen“ [79], eine Körperschaft des öffentlichen Rechts und untersteht der Rechtsaufsicht des Bundesministeriums für Gesundheit (BMG). Der Begriff „Rechtsaufsicht“ ist deutlich zu unterscheiden von einer „Fachaufsicht“. Das bedeutet, dass die Beschlüsse des G-BA inhaltlich nicht vom BMG korrigiert werden können, sondern nur bezüglich formaljuristischer Aspekte. Dies ist ein wesentlicher Aspekt der Unabhängigkeit der Selbstverwaltung im Gesundheitswesen.

Bei seiner Einführung im Jahr 2004 löste der G-BA5 zuvor bestehende Ausschüsse ab: die Bundesausschüsse der Ärzte, Zahnärzte und Krankenkassen, den Ausschuss Krankenhaus sowie die Arbeitsgemeinschaft Koordinierungsausschuss.

Der zentrale Auftrag des G-BA ist, unter Berücksichtigung des Wirtschaftlichkeitsgebots nach $§ 12$ SGB V und unter Einhalten des Leistungsanspruchs nach §2 SGB $\vee$ festzulegen, welche medizinischen Leistungen für die Versicherten von den gesetzlichen Krankenversicherungen getragen werden müssen. Um den in §2 SGB V formulierten Anspruch auf eine qualitativ gute, den medizinischen Fortschritt einbeziehende Behandlung durchzusetzen, sind vom G-BA sowohl für Krankenhäuser als auch für Praxen eine Reihe von Instrumenten geschaffen worden, mit denen die Qualität der erbrachten Leistung gemessen und gesichert werden soll.

Das BMG formuliert den Auftrag des G-BA bezüglich der Qualitätssicherung folgendermaßen:

\begin{abstract}
„Die Festlegung von verbindlichen konkretisierenden Regelungen sowohl im ambulanten als auch im stationären Bereich - also in der vertragsärztlichen Versorgung und in den Krankenhäusern ist dem Gemeinsamen Bundesausschuss (G-BA) übertragen. Das heißt, der G-BA hat die Gestaltungshoheit insbesondere auch für die Qualitätssicherung im Krankenhaus. Er hat somit die Kompetenz zu entscheiden, für welche Bereiche Qualitätsanforderungen bestimmt werden, wie detailliert diese sind und mit welchem Aufwand diese Regelungen verbunden sind. Seine Vorgaben sind für die Leistungserbringer verbindlich" [80].
\end{abstract}

\subsubsection{Struktur des G-BA}

Der G-BA besteht aus dem Plenum, das das beschlussfassende Gremium ist, und 9 Unterausschüssen. Plenum und Unterausschüsse haben eine gleiche Struktur: Sie sind zum einen paritätisch mit Vertretern der Leistungserbringer und Vertretern der Kostenträger besetzt (sog. „Bänke“) und zum anderen mit unparteiischen Mitgliedern, die jeweils auch den Vorsitzenden stellen. Die unparteiischen Mitglieder werden im Konsens der Leistungserbringer und des GKVSpitzenverbandes bestimmt bzw., wenn das nicht gelingt, durch Direktive des BMG. Im Einzelnen ist das Plenum besetzt mit 3 unparteiischen Mitgliedern inklusive des Vorsitzenden, 2 Vertretern der Kassenärztlichen Bundesvereinigung (KBV), einem Vertreter der Kassenzahnärztlicher Bundesvereinigung (KZBV) und 2 Vertretern der Deutschen Krankenhausgesellschaft (DKG), sowie mit 5 Vertretern des Spitzenverbandes der gesetzlichen Krankenkassen 
(GKV Spitzenverband). Als Neuerung gegenüber den vor dem Jahr 2004 bestehenden Ausschüssen sind auch Vertreter akkreditierter Patienten- und Selbsthilfeorganisationen Mitglieder des Plenums und der Unterausschüsse, die ein Antrags- und Mitwirkungsrecht, aber kein Stimmrecht haben (sog. Patientenmitbeteiligung). Die Sitzungen des Plenums sind öffentlich und sollen zukünftig, so der derzeitige Entwurf des MDK-Reformgesetzes, live im Internet übertragen werden und per Mediathek abrufbar sein.

Unterausschüsse sind eingerichtet zu den Themen ambulante spezialfachärztliche Versorgung, Arzneimittel, Bedarfsplanung, Disease-Management-Programme, Psychotherapie, Methodenbewertung, Qualitätssicherung, veranlasste Leistungen und zahnärztliche Behandlung. Die, anders als das Plenum, nicht-öffentlich tagenden Unterausschüsse fassen das Ergebnis ihrer Beratungen als Beschlussempfehlungen an das Plenum zusammen.

\subsubsection{Stellungnahmeverfahren und Stellungnahmeberechtigte}

Der G-BA ist gesetzlich verpflichtet, bei Beratungen, die die Belange Dritter betreffen, diesen die Gelegenheit zur Stellungnahme zu geben. Zu den Stellungnahmeberechtigten gehören unter anderen „Organisationen der Leistungserbringer, Heilberufekammern, Medizinproduktehersteller, pharmazeutische Unternehmen, der Bundesbeauftragte für den Datenschutz und die Informationsfreiheit, das Robert Koch-Institut, die Strahlenschutzkommission oder wissenschaftliche Fachgesellschaften“.

\subsubsection{Finanzierung}

Der G-BA finanziert sich und die beiden ihm zuarbeitenden Institute IQTIG und IQWIG über einen sog. Systemzuschlag, der für jeden stationären Fall (inkl. Selbstzahler) und für jeden ambulanten GKVFall durch die Leistungserbringer von den Kostenträgern erhoben und an das G-BA abgeführt wird [81].

\subsubsection{Themenbereich Qualitätssicherung}

Seine Aktivitäten zum „Themenbereich Qualitätssicherung“, die vom Unterausschuss Qualitätssicherung bearbeitet werden, gliedert der G-BA in 3 Abschnitte [82]:

1. Vorgaben zur Qualitätssicherung

2. Datenerhebung zur Qualitätssicherung

3. Weitere Bereiche der Qualitätssicherung

\subsubsection{Abschnitt 1: Vorgaben zur Qualitätssicherung}

Zur Qualitätssicherung hat der G-BA Vorgaben entwickelt, die das Qualitätsmanagement, Fortbildungspflichten im Krankenhaus, ein einrichtungsübergreifendes Fehlermeldesystem, Mindestmengen, Strukturqualitätsvorgaben und Personalausstattung in Psychiatrie und Psychosomatik betreffen.

\subsubsection{Qualitätsmanagement-Richtlinie (QM-RL)}

In der vom G-BA herausgegebenen „Qualitätsmanagement-Richtlinie“ [83] werden seine Anforderungen an ein „Qualitätsmanagement" beschrieben - das zu unterscheiden ist von der externen Qualitätssicherung (s.3.2.1, 5.2.6.3 und 5.2.6.4.). Es geht hier um ein einrichtungsinternes Qualitätsmanagementsystem mit systematischen und koordinierten Maßnahmen zur Planung, Lenkung,
Kontrolle und kontinuierlichen Verbesserung von Prozessen und Abläufen. Das Qualitätsmanagement soll auch ein Instrument zur Organisationsentwicklung sein. Die primäre Patientenorientierung mit einem besonderen Fokus auf der Patientensicherheit wird besonders betont. Daneben sollen aber auch die Bedürfnisse aller anderen Akteure im Behandlungsprozess berücksichtigt und ihre Zufriedenheit erhöht werden. Weiterhin wird durch den Verweis auf den PDCA-Zyklus implizit Bezug genommen auf Verfahren des Total Quality Management (TQM). Qualitätsziele sollen für alle relevanten Bereiche festgelegt, gemessen und überprüft werden, um dann ggf. notwendige Anpassungen als Teil eines kontinuierlichen Verbesserungsprozesses (KVP) vorzunehmen zu können. Auch die in das Qualitätsmanagement umfassend einzubeziehenden Bereiche - Patientenorientierung einschließlich Patientensicherheit, Mitarbeiterorientierung einschließlich Mitarbeitersicherheit, Prozessorientierung, Kommunikation und Kooperation, Informationssicherheit und Datenschutz, Verantwortung und Führung - entsprechen dem Ansatz des TQM.

Folgende Methoden und Instrumente des Qualitätsmanagements sind verpflichtend anzuwenden: Messen und Bewerten von Qualitätszielen, Erhebung des Ist-Zustandes und Selbstbewertung, Regelung von Verantwortlichkeiten und Zuständigkeiten, Prozess- bzw. Ablaufbeschreibungen, Schnittstellenmanagement, Checklisten und insbesondere OP-Checklisten (Time-out), Teambesprechungen, Fortbildungs- und Schulungsmaßnahmen, Patientenbefragungen, Mitarbeiterbefragungen, Beschwerdemanagement, Patienteninformation und -aufklärung, Risikomanagement, Fehlermanagement und Fehlermeldesysteme (sog. FMS bzw. Critical Incident Reporting Systems, CIRS, s. auch 5.2.5.5 u. 6.10.2). Insbesondere sollen folgende Anwendungsbereiche durch das Qualitätsmanagement geregelt werden: Notfallmanagement, Hygienemanagement, Arzneimitteltherapiesicherheit, Schmerzmanagement und Maßnahmen zur Vermeidung von Stürzen bzw. Sturzfolgen.

Zusammenfassend ist festzustellen, dass der G-BA von den Praxen und Krankenhäusern nicht nur das Vorhandensein eines grundlegenden Qualitätsmanagementsystems, sondern das Vorhandensein eines umfassenden Qualitätsmanagementsystems fordert. Dabei ist den einzelnen Gesundheitsorganisationen die Wahl ihres Qualitätsmanagementsystems explizit freigestellt, sofern es die vom G-BA formulierten Anforderungen erfüllt.

Die Umsetzung eines kleineren Teils der Vorgaben zur Qualitätsmanagement wird bei Krankenhäusern über den jährlichen Qualitätsbericht abgefragt (s. 5.2.6.1). Im vertragsärztlichen ambulanten Sektor werden von den KVen bzw. KZVen Umfragen mit einem standardisierten Fragebogen bei einer zufällig ausgewählten Gruppe der Vertragsärzte durchgeführt, deren Größe mindestens 2,5\% (KVB) bzw. 2 \% (KZVB) der Gesamtzahl der Vertragsärzte umfasst (s. 5.2.6.2).

\subsubsection{Nachweispflichten zur Fortbildung im Krankenhaus}

Der G-BA hat festgelegt, dass seit dem 01.10.2013 auch die Fachärzte im Krankenhaus nachweisen müssen, dass sie ihrer Fortbildungspflicht nachkommen ( $§ 136$ b SGB V) [84]. Innerhalb von 5 Jahren müssen Fortbildungsveranstaltungen besucht werden, die von den Landesärztekammern mit insgesamt mindestens 250 Fortbildungspunkten bewertet sind und die überwiegend fachspezi- 
fisch sein sollen. Die Kontrolle und das Ausstellen der Fortbildungszertifikate wurden an die Landesärztekammern delegiert. Die Fachärzte haben ihre Zertifikate den Krankenhausverwaltungen vorzulegen. Diese müssen dann im jährlichen Qualitätsbericht (s. 5.2.6.1) darlegen, wie hoch der Prozentsatz der Fachärzte mit gültigem Zertifikat an ihrer Einrichtung ist.

\subsubsection{Strukturqualitätsvorgaben}

Der G-BA legt für folgende Behandlungen Mindestanforderungen an die Strukturqualität fest: Behandlung des Bauchaortenaneurysmas, Kinderherzchirurgie, minimalinvasive Herzklappeninterventionen, Positronenemissionstomografie zur Behandlung des nichtkleinzelligen Lungenkarzinoms, Protonentherapie beim Rektumkarzinom, Versorgung von Früh- und Reifgeborenen und Versorgung von Kindern und Jugendlichen mit hämato-onkologischen Krankheiten.

\subsubsection{Mindestmengenregelungen (Mm-R)}

Für elektive stationäre Leistungen, für die laut G-BA ein Zusammenhang zwischen der Durchführungshäufigkeit und Behandlungsqualität besteht, hat der G-BA eine Mindestmengenregelung eingeführt, um nach eigener Aussage zu gewährleisten, dass die durchführenden Ärzte über ausreichende Erfahrung verfügen. Für folgende 8 Leistungen gilt derzeit eine Mindestmengenregelung: Lebertransplantation (inkl. Teilleber-Lebendspende), Nierentransplantation (inkl. Lebendspende), komplexe Eingriffe am Organsystem Ösophagus, komplexe Eingriffe am Organsystem Pankreas, Stammzelltransplantation, Kniegelenk-Totalendoprothesen, koronarchirurgische Eingriffe (derzeit ohne Festlegung einer konkreten Mindestmenge) und Versorgung von Früh- und Neugeborenen mit einem Geburtsgewicht von unter 1250 Gramm (s. Diskussion 5.12.4).

\subsubsection{Anforderungen an einrichtungsübergreifende Fehler- meldesysteme (üFMS)}

Für ein einrichtungsübergreifendes Fehlermeldesystem (üFMS), d. h. nicht ein einrichtungsinternes Fehlermeldesystem, werden Anforderungen an das Betreiben und die Teilnahme gestellt. Einrichtungsübergreifende Fehlermeldesysteme sollen durch das Sammeln von Erfahrungen über Risiken und Fehlerquellen aus vielen verschiedenen Einrichtungen in besonderem Maße zur Vermeidung unerwünschter Ereignisse beitragen. Die Meldungen müssen Anonymität sowohl in Bezug auf den ggf. betroffenen Patienten als auch in Bezug auf die meldende Einrichtung gewährleisten. Die Teilnahme an einem üFMS wird von dem Betreiber bescheinigt, ist im jährlichen Qualitätsbericht zu veröffentlichen und wird mit einem kleinen Betrag pro stationären Fall $(€ 0,20)$ extra vergütet. Beispiele für ein fachspezifisches üFMS sind das „CIRSmedical Anästhesie“ [85], für ein regionales üFMS das „Netzwerk CIRS Berlin“ [86] und für ein überregionales, fachübergreifendes üFMS das „Krankenhaus-CIRS-Netz Deutschland 2.0“ [87].

\subsubsection{Abschnitt 2: Datenerhebung zur Qualitätssicherung}

Für das erklärte Ziel, die „Qualität der medizinischen Versorgung messen, darstellen und vergleichen" zu können, hat der G-BA bereits Verfahren etabliert und ist darüber hinaus dabei, neue Verfahren zu entwickeln, die auch Einfluss auf die Vergütung (in Form von Zu- oder Abschlägen) und die Krankenhaus- und Bedarfsplanung haben sollen.

Qualitätssichernde Maßnahmen werden unterschieden in „einrichtungsübergreifend“, d. h. den Vergleich zwischen verschiedenen Leistungserbringern ermöglichend, in „sektorspezifisch“, d. h. auf einen der Sektoren des Gesundheitswesens bezogen wie z. B. den stationären Sektor oder den ambulanten Sektor, oder als „sektorübergreifend“, d. h. 2 oder mehr Sektoren betrachtend. Zeitlich longitudinale Verfahren werden als „follow-up“ bezeichnet.

Die derzeitigen Qualitätssicherungsverfahren betreffen die Gebiete der Kardiologie und Kardiochirurgie, Gynäkologie und Geburtshilfe, Transplantationsmedizin, orthopädischer Chirurgie sowie Dekubitus, Pneumonie und nosokomiale Infektionen. Für die Hals-Nasen-Ohren-Heilkunde existiert derzeit kein Verfahren. Ein initial vorhandenes Verfahren zur Tonsillektomie wurde wieder eingestellt.

\subsubsection{Qualitätsberichte der Krankenhäuser (Qb-R)}

Seit dem Jahr 2005 sind alle Krankenhäuser gesetzlich dazu verpflichtet, in Qualitätsberichten über ihre Arbeit zu informieren. In der zugehörigen „Regelung zum Qualitätsbericht der Krankenhäuser“ [88] wird als Ziel dieser Maßnahme formuliert, durch eine Verbesserung der Transparenz eine Orientierungs- und Entscheidungshilfe für Patienten und eine Grundlage für vergleichende Informationen und Empfehlungen durch die Kassenärztlichen Vereinigungen und Krankenkassen zu schaffen. Weiterhin wird den Krankenhäusern eine Möglichkeit zur Außendarstellung gegeben. In einer detailliert festgelegten, strukturierten Form werden in dem Bericht Informationen zu den drei Bereichen „Struktur- und Leistungsdaten des Krankenhauses“, „Struktur- und Leistungsdaten der Organisationseinheiten/ Fachabteilungen“ und „Qualitätssicherung“ abgefragt. Der Gesamtumfang bei einem Universitätsklinikum beträgt ca. 1000 Seiten. Neben statistischen Daten z. B. über die Zahl der angestellten Ärzte werden weitere Informationen erhoben zu stationären Patientenzahlen, Art und Zahl der Diagnosen und angebotenen Leistungen, zu verschiedenen Aspekten des Risikomanagements, zur Implementierung eines internen Qualitätsmanagements und zur Teilnahme an der externen Qualitätssicherung sowie zum Vorhandensein von Zielvereinbarungen mit leitenden Ärzten und der Umsetzung der Fortbildungspflicht der Fachärzte. Der Bericht ist jährlich an die gemeinsame Annahmestelle der gesetzlichen Krankenkassen, ihrer Verbände und des Verbands der Privaten Krankenversicherung abzuliefern und wird von diesen veröffentlicht, kann aber auch über die Referenzdatenbank des G-BA abgerufen werden [89]. Wird der Bericht nicht geliefert, wird das Krankenhaus im ersten Jahr auf einer öffentlichen Liste genannt. Als finanzielle Sanktion wird im zweiten Jahr ein Qualitätssicherungsabschlag von $€ 1,00$ pro stationären Fall, im Folgejahr von $€ 2,00$ pro stationären Fall erhoben.

\subsubsection{Berichterstattung zur Umsetzung von Qualitätsma- nagement in der vertragsärztlichen und vertragszahnärztlichen Versorgung}

Im vertragsärztlichen (inkl. MVZ) und vertragszahnärztlichen ambulanten Sektor wird die Umsetzung der Qualitätsmanagementvorgaben des G-BA durch jährliche Erhebungen geprüft, die von den KVen und KZVen durchgeführt werden. Dabei wird eine Stichprobe von mindestens 2,5\% der kassenärztlichen bzw. $2 \%$ der kassenzahnärztlichen Vertragspartner mittels einer Umfrage mit 
einem standardisierten Fragebogen erhoben. Die Angeschriebenen werden zufällig ausgewählt. Die Ergebnisse werden von den Bundesvereinigungen KBV und KZBV in Jahresberichten veröffentlicht $[90,91]$.

\subsubsection{Datengestützte Qualitätssicherungsverfahren (DeQS-RL)}

Durch das neue Verfahren „datengestützte einrichtungsübergreifende Qualitätssicherung“, das auf der im Jahr 2019 in Kraft getretenen Richtlinie DeQS-RL beruht, werden schrittweise mehrere historisch entstandene Qualitätssicherungsverfahren abgelöst bzw. in einer neuen Struktur zusammengefasst [92]. Im Einzelnen betrifft das die sektorenspezifische stationäre und ambulante Qualitätssicherung (s.u.) und die sektorenübergreifende Qualitätssicherung.

Das Erheben und Auswerten der Behandlungsdaten von Patienten soll dadurch vereinheitlicht und sektorenübergreifend zusammengeführt werden, um so belastbarere Erkenntnisse darüber zu gewinnen, in welchen Bereichen es Verbesserungspotenziale gibt und um dadurch sowohl einrichtungsinternes Qualitätsmanagement zu unterstützen, als auch einen kontinuierlichen Qualitätsentwicklungsprozess einzuleiten. Die erhöhte Transparenz des Verfahrens soll sowohl den teilnehmenden Einrichtungen als auch der Patientensicherheit dienen und, durch geeignete Veröffentlichung, der Information und damit der Selbstbestimmung der Patienten.

Drei Qualitätssicherungsverfahren wurden bisher in das neue Verfahren überführt: das „Verfahren 1: Perkutane Koronarintervention und Koronarangiografie“ (QS PCI), das „Verfahren 2: Vermeidung nosokomialer Infektionen - postoperative Wundinfektionen“ (QS WI) und das „Verfahren 3: Cholezystektomie“ (QS CHE). Ab 2020 sollen nach einem Beschluss vom Juni 20193 weitere neue Verfahren eingeführt werden: das „Verfahren 4: Nierenersatztherapie bei chronischem Nierenversagen einschließlich Pankreastransplantationen“ (QS NET), das „Verfahren 5: Transplantationsmedizin“ (QS TX), das die bisherigen transplantationsmedizinischen Leistungsbereiche der QSKH-RL bündelt, und das „Verfahren 6: Koronarchirurgie und Eingriffe an Herzklappen“ (QS KCHK) [93].

Wird ein Patient behandelt, der unter eines der genannten Qualitätssicherungsverfahren fällt, müssen vom Leistungserbringer und von der Krankenkasse die erhobenen Daten in vorgegebener elektronischer Form an die Datenannahmestelle geliefert werden, üblicherweise quartalsweise. Die korrekte Erfassung der Daten muss jährlich mit einer Konformitätserklärung bestätigt werden.

Die Datensätze enthalten neben den eigentlichen QS-relevanten Angaben bzw. Qualitätsdaten (QD) weitere Daten zur Identifikation des Patienten (PID), des Leistungserbringers (LE) und der Krankenkasse (KK) sowie administrative Daten (AD). Exemplarisch werden im Folgenden Details zum Verfahren „QS PCI“ aufgeführt: vom Leistungserbringer werden pro Behandlungsfall 88 Datenmerkmale abgefragt, die für die Auswertung in 20 Qualitätsindikatoren einfließen. Davon sind 2 Indikationsstellungsindikatoren, 11 Prozessindikatoren und 7 Ergebnisindikatoren. Von der Krankenkasse werden administrative Daten, Diagnosen, Prozeduren und Arzneimittelverordnungen abgefragt.

Datenannahmestellen für Krankenhäuser sind die Landesgeschäftsstellen für Qualitätssicherung (LQS), die bei den Landeskrankenhausgesellschaften (LKG) angesiedelt sind, für Vertragsärzte die zuständigen Kassenärztlichen Vereinigungen (KV) oder Kassen- zahnärztlichen Vereinigungen (KZV) und für Krankenkassen ein beauftragtes Institut. Die Datenannahmestellen prüfen die Daten auf Plausibilität, Vollständigkeit und Vollzähligkeit und pseudonymisieren den Leistungserbringer (LE). Danach reichen sie die Daten an die Vertrauensstelle weiter.

Der G-BA hat eine unabhängige, zu diesem Zweck geschaffene „Vertrauensstelle nach § 299 SGB V“ beauftragt, in den entgegengenommenen Daten die Patientenidentifikation (PID) zu pseudonymisieren. Anschließend leitet sie die Daten an die Bundesprüfstelle weiter.

Als Bundesprüfstelle fungiert das „Institut für Qualität und Transparenz im Gesundheitswesen“ (IQTIG). Es führt, falls erforderlich, alle vorhandenen Datensätze eines pseudonymisierten Patienten zusammen und wertet sie einheitlich nach den im jeweiligen Qualitätssicherungs-Verfahren festgelegten, themenspezifischen Kriterien aus. Dadurch werden auch sektorenübergreifende Auswertungen und Verlaufsbetrachtungen möglich. Diese Auswertungen werden an die Landesarbeitsgemeinschaften (LAG) weitergeleitet, sofern es sich nicht um Bundesverfahren handelt. In diesem Fall wird die Auswertung an die Bundesstelle, den Unterausschuss Qualitätssicherung des G-BA, weitergeleitet. Im Regelfall werden Verfahren länderbezogen durchgeführt. Nur bei geringen Fallzahlen oder aufgrund der „Eigenart des Themas“ werden Verfahren bundesbezogen durchgeführt.

Darüber hinaus erstellt das IQTIG anonymisierte Datenauswertungen für den G-BA in Form des jährlichen Bundesqualitätsberichts, der zusätzlich Informationen aus dem Qualitätssicherungsergebnisberichten enthält, die von den LAG überstellt werden (s.u.). Weiterhin erstellt das IQITIG sog. Rückmeldeberichte an die Leistungserbringer, die eine statische Darstellung der Ergebnisse des Leistungserbringers als auch seiner Vergleichsgruppe enthalten. Die Übermittlung erfolgt über die Datenannahmestellen, die den Empfänger für den Versand de-pseudonymisieren.

Die Validität der Daten wird von der Bundesprüfstelle bzw. dem IQTIG geprüft und, im Falle von Leistungserbringerdaten, ggf. auch von den LAG (s.u.). Die Art der Validierung ist verfahrensspezifisch geregelt.

Die Landesarbeitsgemeinschaften (LAG) sind eine für den vorliegenden Zweck neu eingeführte, sektorenübergreifende Struktur, die dem föderalen System der Selbstverwaltung entspricht und schrittweise die Funktion der Landesgeschäftsstellen für Qualitätssicherung (LQS, s. 5.2.6.4) übernehmen wird. Sie werden in jedem Bundesland von Kassenärztlicher Vereinigung (KV), Kassenzahnärztlicher Vereinigung (KZV), Landeskrankenhausgesellschaft (LKG) und Krankenkassen gebildet. Landesärztekammer (LÄK), Landeszahnärztekammer (LZÄK), Vertreter von privaten Krankenkassen und Pflegeberufen werden von der LAG beteiligt, Patientenvertreter haben ein Mitberatungsrecht. Die LAG richtet Fachkommissionen mit Expertise für das jeweilige QS-Verfahren ein. Nach Erhalt der Daten durch die Bundesauswertungsstelle werden sie von den Fachkommissionen danach bewertet, ob Auffälligkeiten vorliegen. Die LAG hat den Auftrag, qualitätssichernde Maßnahmen einzuleiten, falls Auffälligkeiten festgestellt wurden (auffällig können auch überdurchschnittlich gute Ergebnisse sein). Dazu erfolgt zunächst die De-Pseudonymisierung des Leistungserbringers gegenüber der LAG (bei Bundesverfahren gegenüber der Bundesstelle) durch die Datenannahmestelle. 
Danach wird ein mehrstufiges Verfahren zur Qualitätssicherung eingeleitet: Zunächst erhält der Leistungserbringer Gelegenheit, Stellung zu nehmen. Das kann schriftlich und darüber hinaus auch durch Gespräche erfolgen (sog. Stellungnahmeverfahren). Falls sich dadurch die Auffälligkeiten nicht klären lassen, wird mit dem Leistungserbringer eine Vereinbarung geschlossen, die Maßnahmen der Stufe 1 enthält wie z. B. Teilnahme an geeigneten Fortbildungen, Fachgesprächen, Kolloquien, Teilnahme an Qualitätszirkeln, Implementierung von Behandlungspfaden, Durchführung von Audits, Durchführung von Peer Reviews, Implementierung von Handlungsempfehlungen anhand von Leitlinien usw.. Wenn diese Maßnahmen nicht zum Erfolg führen oder verweigert werden oder wenn „schwerwiegende einzelne Missstände“ vorliegen, wird der Leistungserbringer zur „Äußerung“ aufgefordert. Bei unzureichendem Ergebnis werden Maßnahmen der Stufe 2 beschlossen, die neben einer Korrektur der geschlossenen Vereinbarung als schwerste Sanktion Empfehlungen an die KV/KZV bzw. die Krankenkassen enthalten, Vergütungsabschläge oder das Entziehen der Abrechnungsmöglichkeit durchzuführen. Bei „besonders schwerwiegenden Missständen“, im Wiederholungsfall oder bei Nichtnachkommen der Dokumentationspflicht können direkt die Maßnahmen der Stufe 2 zur Anwendung kommen. Krankenhäuser müssen die Bewertung ihrer auffälligen Befunde im strukturierten Qualitätsbericht veröffentlichen.

Die LAG fassen die Ergebnisse der Bearbeitung von Auffälligkeiten und der ggf. daraus folgenden qualitätssichernden Maßnahmen im „Qualitätssicherungsergebnisbericht“ zusammen und erstellt zur Information der Öffentlichkeit einen laienverständlichen Bericht.

\subsubsection{Externe stationäre Qualitätssicherung (QSKH-RL)}

Die „externe stationäre Qualitätssicherung“ ist das bisherige externe Qualitätssicherungsverfahren entsprechend der Richtlinie über „Maßnahmen der Qualitätssicherung an Krankenhäusern“ (QSKH-RL) [94], das schrittweise durch die zuvor besprochenen „Datengestützten Qualitätssicherungsverfahren“ (nach DeQS-RL) abgelöst werden wird. Im Gegensatz zu dem neuen Verfahren gibt es bei den Falldatensätzen nach QSKH-RL keine Patientenidentifikation, sondern nur Angaben zu Geburtsjahr und Alter des Patienten bei Aufnahme und Entlassung. Damit ist eine fall- oder sektorenübergreifende Zusammenführung von Daten nicht möglich, andererseits entfällt aber auch die aufwendige Pseudonymisierung des neuen Verfahrens. Auch werden bisher keine Daten von den Krankenkassen abgefragt. Analog zum neuen Verfahren gibt es eine Unterscheidung von landes- und bundesbezogenen Verfahren, die allerdings mit einer anderen Terminologie als „direkt“ ( = bundesbezogen) und „indirekt“ ( = landesbezogen) bezeichnet werden. Die überwiegende Zahl der Verfahren gehört zu der Gruppe der indirekten Verfahren. Direkte Verfahren sind derzeit z. B. die Transplantationsverfahren.

Eine zentrale Rolle bei der Annahme der Daten, Weiterleitung an das IQTIG und Bewertung von rechnerischen Auffälligkeiten, spielen die den Landeskrankenhausgesellschaften (LKG) zugeordneten Landesgeschäftsstellen für Qualitätssicherung (LQS) [95]. Die in ihnen gebildeten Strukturen - Lenkungsgremium, Fachgruppen, Landesgeschäftsstelle - finden sich in ähnlicher Weise in den für das neue Verfahren neu geschaffenen Landesarbeitsgemein- schaften (LAG) wieder, die allerdings anders als die LQS organisatorisch von den LKG unabhängig sind. Das Lenkungsgremium der LQS ist mit Vertretern der Landesverbände der Krankenkassen und Ersatzkassen, der Landeskrankenhausgesellschaft, der Landesärztekammer, des Landespflegerats und von Patientenorganisationen besetzt. Die Fachgruppen der LQS sind jeweils einem Qualitätssicherungsverfahren zugeordnet und mit ärztlichen und pflegerischen Experten besetzt, die von den Spitzenverbänden der Ärztekammern, der AWMF, dem Pflegerat und den gesetzlichen und privaten Kassen entsandt werden.

Eine Validierung der übermittelten Daten findet statt durch eine statistische Basisprüfung, anlassbezogene Datenabgleiche mit Patientenakten und durch anlasslose, mit dem IQTIG koordinierte Stichproben in 3 Leistungsbereichen pro Jahr.

Nachdem die Daten in den „indirekten Verfahren“ von den LQS angenommen wurden, werden sie an das IQTIG weitergereicht, von dem sie bezüglich des Standortes pseudonymisiert werden, um eine objektive Beurteilung durch die LQS zu gewährleisten, nachdem sie zu diesen zurückgereicht wurden. Sollten sich bei den LQS bei der Bewertung der von einem Krankenhaus gemeldeten Qualitätsindikatoren rechnerische Auffälligkeiten ergeben, dann leitet die LQS einen sog. „strukturierten Dialog“ ein. Dieser ist ein mehrstufiges Verfahren, das immer beim Auftreten von Auffälligkeiten ausgelöst werden muss, und stellt eine wesentliche Aufgabe der LQS dar. Unter Hinzuziehen der Expertise der zuständigen Fachgruppe kann das auffällige Ergebnis als nicht-plausibel und damit ohne Konsequenz bewertet werden, oder es kann zu Maßnahmen führen. In letzterem Fall erfolgt eine De-Pseudonymisierung des Krankenhauses durch das IQTIG. Die geringste Maßnahme ist ein „Hinweis“ an das Krankenhaus, bei dem keine Antwort erwartet wird. Danach kommt stufenweise das Anfordern einer Stellungnahme, einer Besprechung, einer Begehung und einer Zielvereinbarung. An jedem Punkt dieser eskalierenden Maßnahmen kann die LQS den strukturierten Dialog als beendet erklären, wenn nach seiner Überzeugung der gewünschte Effekt bereits eingetreten ist. Verweigert ein Krankenhaus die Teilnahme am strukturierten Dialog, erfüllt es die Zielvereinbarung nicht oder kommt es seiner Verpflichtung zur Datenübermittlung nicht nach („erhebliche Dokumentationsmängel“), wird es an das Lenkungsgremium gemeldet, bzw. beim direkten Verfahren an den Unterausschuss Qualitätssicherung des G-BA. Die auf ein Scheitern des strukturierten Dialogs folgenden Sanktionen sind in der Richtlinie nicht spezifiziert. Es sind lediglich Sanktionen für Dokumentationsmängel als sog. "Qualitätssicherungsabschläge“ festgelegt, die je nach Verfahren und Schwere des Mangels pro Fall zwischen $€ 150$ und $€ 5000$ betragen können.

Die Finanzierung der Aufwände für die Qualitätssicherung sowohl in den Krankenhäusern als auch in den LQS auf Landesebene erfolgt über einen sog. Qualitätssicherungszuschlag, der auf jeden stationären Fall von den Leistungsträgern erhoben wird.

\subsubsection{Qualitätsreport}

Im aktuellen Qualitätsreport des IQTIG aus dem Jahr 2018 wird über die Ergebnisse der externen Qualitätssicherung aus dem Jahr 2017 sowie über die Ergebnisse des strukturierten Dialogs aus dem Jahr 2016 berichtet [96]. In 26 verschiedenen Qualitätssicherungsverfahren (24 stationären und 2 sektorenübergreifenden) wurden ca. 
$25 \%$ aller stationären Leistungen geprüft. Für die 24 stationären Qualitätssicherungsverfahren haben 1516 Krankenhäuser rund 2,5 Mio. Datensätze übermittelt, für die 2 sektorenübergreifenden Qualitätssicherungsverfahren haben 273 vertragsärztlichen Praxen oder Medizinischen Versorgungszentren und 1063 Krankenhausstandorte ca. 0,8 Mio. Datensätze übermittelt, d. h. insgesamt wurden ca. 3,3 Mio. Datensätze erfasst. Bei 4\% der in diesen Verfahren erfassten Qualitätsindikatoren wurde wegen „ausgeprägter oder fortgesetzter Qualitätsdefizite“ ein „besonderer Handlungsbedarf" festgestellt.

Die Ergebnisse des strukturierten Dialogs beziehen sich auf das Jahr 2016. Aus 2482141 übermittelten Datensätzen wurden 116163 Ergebnisse, d. h. die Gesamtzahl der bewerteten Verfahren an allen Standorten, berechnet. Davon waren 12683 (10,9\%) rechnerisch auffällig. Von diesen wurden 7607 (60\%) im strukturierten Dialog überprüft, an die übrigen $40 \%$ wurde meist ein Hinweis gesandt. 1611 Datensätzen wurde nach Überprüfung durch die LQS als qualitativ auffällig eingestuft. Das sind $12,7 \%$ der rechnerisch auffälligen Ergebnisse oder 1,0\% aller berechneten Ergebnisse. Die übrigen Ergebnisse wurden als unauffällig bewertet oder waren wegen schlechter Dokumentationsqualität nicht bewertbar. In der Folge wurden 7607 Stellungnahmen angefordert, 275 Besprechungen durchgeführt, 13 Begehungen durchgeführt und 742 Vereinbarungen getroffen

\subsubsection{Externe ambulante Qualitätssicherung}

Für den ambulanten Bereich gibt es derzeit nur ein Verfahren der externen Qualitätssicherung. Dieses bezieht sich auf die Dialyse (Qualitätssicherungs-Richtlinie Dialyse/QSD-RL) [97]. Zukünftig wird das ambulante Verfahren nach QSD-RL in das neue sektorenübergreifende „Verfahren 4: Nierenersatztherapie bei chronischem Nierenversagen einschließlich Pankreastransplantationen “ nach NET-RL überführt werden.

\subsubsection{Sekundäre Datennutzung}

Zum Zweck der Forschung oder der Weiterentwicklung der Qualitätssicherung können Dritte auf Antrag an das IQTIG Daten aus der datengestützten Qualitätssicherung nutzen.

\subsubsection{Qualitätsindikatoren für die Krankenhausplanung (planQI-RL)}

Im Krankenhausstrukturgesetz wurde im Jahr 2016 erstmals festgelegt, dass bei der Krankenhausplanung die Qualität der erbrachten Leistungen eines Krankenhauses eine Rolle spielen soll. Der G-BA wurde beauftragt, zu diesem Zweck Qualitätsindikatoren zu entwickeln und anzuwenden, um durch die daraus folgende Bewertung den politischen Entscheidungsgremien auf Landesebene Entscheidungshilfen für die Krankenhausplanung an die Hand zu geben. Allerdings sind die Länder nicht verpflichtet, diese Kriterien auch anzuwenden, d. h. sie sind durch Landesrecht auch ausschließbar. Derzeit haben Bayern, Nordrhein-Westfalen, Rheinland-Pfalz, Mecklenburg-Vorpommern, Niedersachsen, Hessen und BadenWürttemberg von dieser Möglichkeit des Opt-out Gebrauch gemacht, Thüringen hat es vor (s. 5.12.2). Dies wurde vom Vorsitzenden des G-BA, Prof. Josef Hecken, scharf kritisiert [98].

In der Richtlinie zu planungsrelevanten Qualitätsindikatoren planQI-RL wird das Verfahren festgelegt [99]. Angesichts der Trag- weite des Verfahrens sei ein besonderes Gewicht auf die Auswahl relevanter, aussagekräftiger Qualitätsindikatoren gelegt worden. Im Unterschied zur externen Qualitätssicherung nach QSKH-RL werden die Datensätze bundesweit direkt vom IQTIG ausgewertet. Rechnerisch auffällige, d. h. außerhalb des zuvor definierten Referenzbereichs liegende Ergebnisse, werden darauf geprüft, ob sie auch statistisch signifikant auffällig sind. In diesem Fall wird ein Stellungnahmeverfahren eingeleitet und gleichzeitig bei den LQS nachgefragt, ob es für die betroffene Klinik Ergebnisse aus einem zurückliegenden strukturierten Dialog gibt. Die Stellungnahme des Krankenhauses und ggf. die Antwort der LQS werden von einer Fachkommission am IQTIG, die aus gepoolten, berufenen Mitgliedern der bei den LQS eingerichteten Fachgruppen besteht, auf die Frage bewertet, ob ein „Ausnahmetatbestand“ vorliegt, der eine negative Bewertung vermeiden würde. Ist dies nicht der Fall, wird dem Krankenhaus für die geprüfte Leistung eine „qualitativ unzureichende Leistung“ attestiert. Dies muss das Krankenhaus in seinem jährlichen Qualitätsbericht so veröffentlichen. Gleichzeitig werden die für die Krankenhausplanung zuständigen Landesbehörden und die Landesverbände der Krankenkassen informiert, inkl. der Weiterleitung der vom Krankenhaus abgegebenen Stellungnahme. Weiterhin werden die bundesweiten Ergebnisse vom G-BA veröffentlicht. Derzeit wird dieses Verfahren bereits in den 3 Bereichen gynäkologische Operationen, Geburtshilfe und Mammachirurgie unter Beurteilung von elf Qualitätsindikatoren angewendet. Ein erster Bericht dazu wurde im Jahr 2018 veröffentlicht [100]. Einige der betroffenen Kliniken haben daraufhin die als „qualitativ unzureichend“ bewerteten Leistungen eingestellt [101].

Die AWMF sieht die gesetzlichen Vorgaben für planungsrelevante Qualitätsindikatoren und qualitätsabhängige Vergütung (siehe nächster Abschnitt) in der jetzigen Ausgestaltung kritisch [102] (s. Diskussion 5.12).

\subsubsection{Qualitätsabhängige Vergütung}

Der G-BA wurde von Gesetzgeber beauftragt, Leistungsbereiche mit dazugehörigen geeigneten Qualitätsindikatoren zu identifizieren, für die Krankenhäuser und Krankenkassen zukünftig für besonders gute Qualität Leistungszuschläge und für unzureichende Qualität Leistungsabschläge vereinbaren sollen können. Auch hier soll das IQTIG eine zentrale Rolle spielen. Derzeit sind solche Leistungsbereiche und Qualitätsindikatoren noch nicht benannt worden. Lediglich die für die Teilnahme an einrichtungsübergreifenden Fehlermeldesystemen (üFMS, s. 5.2.5.5) zu beanspruchenden Leistungszuschlägen können als eine Art qualitätsabhängiger Vergütung eingeordnet werden (s. Diskussion 5.2.6.9).

\subsubsection{Qualitätsprüfung und -beurteilung im ambulanten Bereich (QP-RL)}

Die Überprüfung der Qualität von niedergelassenen Vertragsärzten in den Bereichen radiologische Diagnostik und Computertomografie, Kernspintomografie und Arthroskopie wird entsprechend der Richtlinie QP-RL von den KVen durch Stichproben durchgeführt. Dabei müssen die Vertragsärzte zu den genannten Bereichen eine vollständige Dokumentation zur Überprüfung vorlegen, die durch Einzelrichtlinien festgelegt ist. Bei der Arthroskopie muss z. B. der endoskopische Befund vor und nach therapeutischer Intervention 
lückenlos dokumentiert sein. Die KVen übermitteln die Ergebnisse ihrer Prüfungen jährlich an die KBV, die sie ihrerseits in einem Bericht an die G-BA zusammenfasst.

Da die QP-RL aufgrund einer Gerichtsentscheidung wegen fehlender Pseudonymisierung der Patientenidentifikation gerade grundlegend überarbeitet worden ist und die Richtlinien für die 3 o.g. Bereiche noch nicht angepasst worden sind, wurde die Stichprobenprüfung seit dem zweiten Quartal 2019 ausgesetzt [103].

\subsubsection{Abschnitt 3: Weitere Bereiche der Qualitätssicherung}

\subsubsection{Qualitätskontrollen des Medizinischen Dienstes der} Krankenversicherung

Durch das Krankenhausstrukturgesetz ist der MDK beauftragt worden, die bei Qualitätssicherungsverfahren ggf. notwendig werdenden Überprüfungen in Krankenhäusern für den G-BA durchzuführen. Das Verfahren ist durch die MDK-Qualitätskontroll-Richtlinie (MDKQK-RL) [104] des G-BA geregelt. Es kommt zur Anwendung, um das Einhalten von Qualitätsanforderungen und die korrekte Dokumentation zu überprüfen, und kann direkt vom G-BA, den mit der Qualitätssicherung befassten Gremien auf Bundes- und Landesebene und den Krankenkassen veranlasst werden. Der Anlass für eine Beauftragung des MDK zur Qualitätskontrolle kann gegeben sein im Rahmen von Verfahren zur externen Qualitätssicherung bzw. Erhebung von Qualitätsindikatoren in ähnlichen Verfahren, wegen Auffälligkeiten im Qualitätsbericht oder in der Abrechnung gegenüber den Krankenkassen, wegen Dokumentationsmängeln und ernsthaften, ggf. wiederholten Hinweisen von Dritten wie z. B. Versicherten. Das Verfahren wird üblicherweise als angekündigte Kontrolle vor Ort durchgeführt. In begründeten Ausnahmefällen kann es auch als unangekündigte Kontrolle vor Ort, oder bei geringerer Relevanz schriftlich erfolgen. Das Krankenhaus hat eine Mitwirkungspflicht. Der MDK berichtet über die Ergebnisse als Kontrollbericht an die beauftragende Stelle. Sollte er erhebliche, d. h. z. B. lebensgefährliche Qualitätsmängel feststellen, ist er zum Erstatten einer sofortigen Mitteilung nicht nur an den Auftraggeber, sondern auch an Dritte (zuständigen Gesundheitsbehörden der Länder und kommunale Gesundheitsämter) verpflichtet. Gleichzeitig gehen Berichte und Mitteilungen immer auch an das betroffene Krankenhaus.

\subsubsection{Regelungen von Folgen bei der Nichteinhaltung von Qualitätsvorgaben (QFD-RL)}

Der G-BA ist nach §137 SGB V damit beauftragt, ein System von Konsequenzen aus dem Nichteinhalten von Qualitätsanforderungen zu entwickeln. Dieses System soll in ansteigender Eskalationsreihe „Vergütungsabschläge, den Wegfall des Vergütungsanspruchs für Leistungen, bei denen Mindestanforderungen nicht erfüllt sind, die Information Dritter über die Verstöße und die einrichtungsbezogene Veröffentlichung von Informationen zur Nichteinhaltung von Qualitätsanforderungen“ enthalten. Der Auftrag schließt weiterhin ein, dass die „Maßnahmen verhältnismäßig zu gestalten und anzuwenden“ sind. Der G-BA hat hierzu 2019 eine grundsätzliche Qualitätsförderungs- und Durchsetzungs-Richtlinie (QFD-RL) [105] beschlossen. Dem Durchsetzungsteil, der die o.g. Konsequenzen beinhaltet, ist ein Förderungsteil vorangestellt. Damit soll beim Vorliegen von Qualitätsmängeln zunächst versucht werden, durch eine schriftliche Vereinbarung über die Teilnahme an Maßnahmen die Qualitätsmängel zu beseitigen. Folgende 12 Maßnahmen werden in der Richtlinie genannt: „1. Schriftliche Empfehlung, 2. Zielvereinbarung, 3. Teilnahme an geeigneten Fortbildungen, Fachgesprächen, Kolloquien, 4. Teilnahme an Qualitätszirkeln, 5. Teilnahme an Audits, 6. Begehungen/Visitationen, 7. Teilnahme an Peer Reviews, 8. Implementierung von Vorgaben für das interne Qualitätsmanagement, 9. Implementierung von Behandlungspfaden, 10. Implementierung von Standard Operating Procedures (SOPs), 11. Implementierung von Handlungsempfehlungen anhand von Leitlinien und 12. Prüfung unterjähriger Auswertungsergebnisse“.

Das Umsetzen dieser allgemeinen Richtlinie ist laut G-BA von der Konkretisierung in den themenspezifischen Richtlinien abhängig und tritt deshalb auch erst dann in Kraft, wenn dieser Schritt erfolgt ist.

\subsubsection{Leistungsbereiche zur Erprobung von Qualitätsverträgen}

Gemäß § 136b Absatz 1 Satz 1 Nummer 4 SGB V hat der G-BA den Auftrag, 4 Bereiche zu benennen, für die Krankenhäuser und Krankenkassen zeitlich befristet Verträge mit einem Anreizsystem für besondere Qualitätsanforderungen schließen können. Der G-BA hat nach eigener Aussage einen Bereich mit erkennbarem Verbesserungspotenzial, die endoprothetische Gelenkversorgung, und 3 Bereiche mit „,besonders vulnerablen Patienten“, nämlich die „Prävention des postoperativen Delirs bei der Versorgung von älteren Patienten, die Respiratorentwöhnung von langzeitbeatmeten Patienten sowie die Versorgung von Menschen mit geistiger Behinderung oder schweren Mehrfachbehinderungen im Krankenhaus“ dafür ausgewählt [106]. Über konkret abgeschlossene Verträge liegen weder von der G-BA, der DKG noch dem Spitzenverband der GKV Informationen vor.

\subsubsection{Andere Themenbereiche bzw. Unterausschüsse des G-BA}

Auch alle anderen Unterausschüsse des G-BA (s. 5.2.1) beschäftigen sich mit Themen, die zumindest implizit Qualitätsaspekte haben, da neben dem Wirtschaftlichkeitsgebots nach §12 SGB V immer auch der Anspruch nach §2 SGB V auf eine qualitativ gute, den medizinischen Fortschritt einbeziehende Leistung berücksichtigt werden soll.

\subsubsection{Frühe Nutzenbewertung}

Der bekannteste Unterausschuss des G-BA ist der zum Thema Arzneimittel und hierbei insbesondere zur „Frühen Nutzenbewertung“ von neuen Arzneimitteln auf Grundlage des §35a SGB V und des Gesetzes zur Neuordnung des Arzneimittelmarktes (AMNOG) von 2011. Bei der Bewertung des Nutzens neuer Arzneimittel, bei dem intensiv das andere dem G-BA zuarbeitende „Institut für Qualität und Wirtschaftlichkeit im Gesundheitswesen“ (IQWIG) eingebunden ist, wird auf die 4 Aspekte Morbidität, Mortalität, Nebenwirkungen und Lebensqualität - als valideste Form eines Patient Reported Outcome (PRO, s. 3.4) - abgestellt. Insbesondere zu dem letzten Punkt gibt es intensive Diskussionen, da von Seiten der pharmazeutischen Industrie und dritter Seite das Insistieren auf der mit validierten Instrumenten erhobenen Lebensqualität in Frage gestellt wird und andere PRO-Methoden wie subjektive Nutzenbewertungen durch die Patienten, Schmerzskalen usw. als ebenbür- 
tig dargestellt werden [107]. Von der AWMF wurde in einer aktuellen Stellungnahme kritisiert, dass Medikamente für chronische Erkrankungen schlechter bewertet werden als Medikamente für Erkrankungen mit kurzer Lebenserwartung und Orphan Drugs. Es sei eine „stärkere Berücksichtigung von Parametern der Morbidität bei chronisch verlaufenden Erkrankungen“ erforderlich. Die „sensitive, umfassende Erfassung der Lebensqualitätsänderungen in allen Zulassungsstudien “ wird hingegen ausdrücklich unterstützt [108] (s. auch 5.4.2 und [109]).

\subsubsection{Erprobungsrichtlinie Tonsillotomie}

Unser Fachgebiet betreffend hat der Unterausschuss Methodenbewertung im Jahr 2018 die „Richtlinie zur Erprobung der Tonsillotomie bei rezidivierender akuter Tonsillitis“ (ErpRL Tonsillotomie) [110] beschlossen, in der eine Multi-Center-Studie zum Vergleich von Effektivität und Nebenwirkungen von Tonsillotomie und Tonsillektomie bei rezidivierenden akuten Tonsillitiden beauftragt wird. In der Begründung wird angeführt, dass die Tonsillotomie bei „Verringerung der periprozeduralen Belastung gegenüber der Tonsillektomie“ das Potenzial einer Behandlungsalternative hat, derzeit aber keine ausreichende Studienlage zur definitiven Beurteilung dieser Frage vorliegt.

\subsection{IQTIG - Institut für Qualität u. Transparenz im Gesundheitswesen}

Von den 2, dem G-BA zuarbeitenden Instituten ist das Institut für Qualität und Transparenz im Gesundheitswesen (IQTIG) das entscheidende in Qualitätsfragen.

\subsubsection{Organisation}

Durch das GKV-Finanzstruktur- und Qualitätsweiterentwicklungsgesetzes (GKV-FQWG), das am 1. Januar 2015 in Kraft getreten ist, wurde in das SGB V der §137a [111] eingebracht, auf dessen Grundlage das IQTIG als fachlich unabhängiges, wissenschaftliches Institut gegründet wurde. Mit der Durchführung war der G-BA beauftragt, der zu diesem Zweck eine Stiftung des privaten Rechts zu errichten, die Gründerin und Trägerin des Instituts ist. Die Gremien der Stiftung und des IQTIG sind paritätisch mit Vertretern der Leistungserbringer und der Krankenkassen besetzt.

Das IQTIG übernahm die Aufgaben des zuvor damit betrauten privaten AQUA-Instituts [112], das es zum 01.01.2016 ablöste.

\subsubsection{Aufgaben}

Die Aufgaben des IQTIG wurden zum Teil schon in den vorangegangenen Abschnitten umfassend dargestellt (s. 5.2.4). Den Gesetzestext nach §135a Absatz 3 SGB V zur Beschreibung der Aufgaben des IQTIG fasst das Institut folgendermaßen zusammen:

- „Erarbeitung von Instrumenten der Qualitätssicherung, Darstellung der Versorgungsqualität im Gesundheitswesen und Mitwirkung an deren Umsetzung - im Auftrag des G-BA.

- Fortführung und Weiterentwicklung der bereits existierenden Qualitätssicherungsverfahren.

- Entwicklung und Durchführung von Verfahren, um die externe Qualitätssicherung in der stationären und ambulanten Versorgung besser zu verzahnen. Parallel dazu erarbeitet das IQTIG im Auftrag des G-BA methodische Grundlagen dafür, dass die
Landesbehörden bei der Krankenhausplanung die Versorgungsqualität von Kliniken berücksichtigen können.

- Schaffung von Kriterien zur Bewertung von Zertifikaten und Qualitätssiegeln im ambulanten wie stationären Bereich.

- Publizierung der Arbeitsergebnisse in einer für die Allgemeinheit verständlichen Form. Dazu gehört auch der Aufbau einer Internetseite, die es Patientinnen und Patienten ermöglichen soll, Krankenhäuser hinsichtlich ihrer Qualität miteinander zu vergleichen.

- Nach dem Krankenhausstrukturgesetz hat das IQTIG u. a. die Aufgabe, im Auftrag des G-BA Konzepte für

- Planungsrelevante Qualitätsindikatoren,

- Zu- und Abschläge in der qualitätsorientierten Vergütung und

- die Evaluation der Qualitätsverträge nach § 110a SGB V zu erarbeiten“ [113].

Der Gesetzgeber schreibt ausdrücklich vor, dass „bei der Entwicklung der Inhalte“ alle relevanten Vertreter des deutschen Gesundheitswesens zu beteiligen sind ( $§ 137$ a Absatz 7 SGB V).

Neben der direkten Beteiligung an Verfahren der externen Qualitätssicherung, Bedarfsplanung und qualitätsbezogener Vergütung besteht eine weitere wichtige Aufgabe des IQTIG darin, die Methoden für die o.g. Anwendungen auf wissenschaftlicher Grundlage weiterzuentwickeln. Es handelt sich um einen iterativen Prozess, bei dem ein Entwurf des IQTIG den stellungnahmeberechtigten Organisationen zugesandt wird, aufgrund dieser Stellungnahmen überarbeitet und erneut zur Stellungnahme versandt wird [14, 114-118].

Ein besonderer Aspekt der Arbeit des IQTIG ist das Entwickeln von Instrumenten zur Patientenbefragung, die die Perspektive des „Patient reported outcome“ (PRO, s. 3.4) in die Qualitätsbetrachtung mit einbringen soll. Sie werden in der Beschreibung der „Methodischen Grundlagen“ unterschieden in „PROM“ (patient reported outcome measures) und „PREM“ (patient reported experience measures) [14], stellen also keine Instrumente zum standardisierten Erfassen der Lebensqualität (QoL, s. 3.4) dar. Für die Bereiche Herzkatheter/Stent und Schizophrenie existieren diese Patientenbefragungen bereits, für die Bereiche Nierenersatztherapie und ambulante Psychotherapie werden sie gerade entwickelt.

Seinen Publikationsverpflichtungen kommt das IQTIG durch eine Reihe von Veröffentlichungen nach, so z. B. den Qualitätsreport [96] (s. 5.2.6.5), die Bundesauswertung, den Bundesqualitätsbericht, den Bericht zum Strukturierten Dialog, den Bericht zur Datenvalidierung, den Strukturierten Qualitätsbericht, das Validierungsverfahren NICU (Neonatal Intensiv Care Unit), Entwicklungsberichte, Evaluationsberichte, Sonderauswertungen und letztlich seinen jährlichen Tätigkeitsbericht [119].

\subsection{IQWIG - Institut für Qualität u. Wirtschaftlichkeit im Gesundheitswesen}

Das Institut für Qualität und Wirtschaftlichkeit im Gesundheitswesen (IQWIG) ist ein sog. HTA (Health Technology Assessment) -Institut [120]. Nachdem in anderen europäischen Ländern, insbesondere Skandinavien, schon seit mehr als 10 Jahren HTA-Institute bestanden hatten, wurde in Deutschland im Jahr 2004 das IQWIG gegründet. 


\subsubsection{Organisation}

Auf der Grundlage des GKV-Modernisierungsgesetzes (s.5.1.1) und der dadurch eingebrachten § 139a, § 139b, § 139c SGB V wurde im Jahr 2004 eine gleichnamige Stiftung ins Leben gerufen mit dem Auftrag, das IQWIG zu gründen. Der Vorstand besteht aus 5 Mitgliedern, von denen einer vom BMG, 2 vom Spitzenverband GKV und je ein weiterer von KBV und DKG gestellt werden. Die Aufsicht über den Vorstand führt der Stiftungsrat, der paritätisch von Vertretern der Leistungserbringern und des Spitzenverbandes der GKV besetzt ist. Dem Vorstand zur Seite gestellt ist neben dem wissenschaftlichen Beirat ein Kuratorium, in dem neben Vertretern der direkten Akteure im Gesundheitswesen auch Vertreter gesellschaftlicher Gruppen und der pharmazeutischen Industrie vertreten sind. Das Kuratorium kann Beschlüsse des IQWIG durch Stellungnahmen kommentieren. Die Leitung des Instituts wird durch den Vorsitzenden und seinen Stellvertreter ausgeübt, die durch sog. Stabsbereiche unterstützt werden. Die „berichterstellenden Ressorts “ zu den Bereichen Arzneimittelbewertung, Nichtmedikamentöse Verfahren, Versorgung und Gesundheitsökonomie und Medizinische Biometrie liefern die Grundlage für die Empfehlungen des IQWIG.

\subsubsection{Aufgaben}

Das derzeitige Aufgabenspektrum ist seit der Gründung durch eine Reihe von Gesetzen und Verordnungen fortgesetzt erweitert und modifiziert worden. Generell besteht die Aufgabe des IQWIG darin, Vor- und Nachteile medizinischer Leistungen möglichst unter Anwendung der Methodik evidenzbasierter Medizin so objektiv wie möglich zu überprüfen und zu bewerten.

Den Auftrag dazu erhält das IQWIG vom G-BA oder dem BMG, es kann darüber hinaus auch eigeninitiativ oder auf Anregung von Seiten der Allgemeinheit tätig werden. Beurteilungen, die das Institut im Auftrag des G-BA erstellt hat, leitet es ihm als Empfehlungen zu, die der G-BA laut Gesetz bei seiner Entscheidungsfindung zu berücksichtigen hat. Gegenstand der Beurteilung sind u.a. Arzneimittel, nichtmedikamentöse Behandlungsmethoden wie z. B. Operationsmethoden, Screeningverfahren und Disease-Management-Programme (DMP). Darüber hinaus erstellt das Institut evidenzbasierte Gesundheitsinformationen für Laien. Die genaue Vorgehensweise ist publiziert in dem Methodenpapier „Allgemeine Methoden 5.0“ [121].

Im Gesetzestext werden die Aufgaben im § 139a SGB V im Detail festgelegt:

- „Recherche, Darstellung und Bewertung des aktuellen medizinischen Wissensstandes zu diagnostischen und therapeutischen Verfahren bei ausgewählten Krankheiten,

- Erstellung von wissenschaftlichen Ausarbeitungen, Gutachten und Stellungnahmen zu Fragen der Qualität und Wirtschaftlichkeit der im Rahmen der gesetzlichen Krankenversicherung erbrachten Leistungen unter Berücksichtigung alters-, geschlechts- und lebenslagenspezifischer Besonderheiten,

- Bewertungen evidenzbasierter Leitlinien für die epidemiologisch wichtigsten Krankheiten,

- Abgabe von Empfehlungen zu Disease-Management-Programmen, Bewertung des Nutzens und der Kosten von Arzneimitteln, Bereitstellung von für alle Bürgerinnen und Bürger verständlichen allgemeinen Informationen zur Qualität und Effizienz in der
Gesundheitsversorgung sowie zur Diagnostik und Therapie von Krankheiten mit erheblicher epidemiologischer Bedeutung,

- Beteiligung an internationalen Projekten zur Zusammenarbeit und Weiterentwicklung im Bereich der evidenzbasierten Medizin“.

Mit dem GKV-Wettbewerbsstärkungsgesetz (GKV-WSG, s. 5.1.2) wurde im Jahr 2007 die Kosten-Nutzen-Bewertung (KNB) für Arzneimittel nach §35b SGB V eingeführt. Das IQWIG entwickelte dazu mithilfe internationaler Experten das Instrument „Analyse der Effizienzgrenze“ [122], durch das das Abschätzen angemessener Preise bei neuen Medikamenten mit Zusatznutzen möglich wurde.

Durch das Arzneimittelmarktneuordnungsgesetz (AMNOG, s. 5.1.4) im Jahr 2011 wurde die Preisfindung auf ein neues Verfahren, die sog „frühe Nutzenbewertung“ verlagert. Die KNB ist seitdem ein Reserveverfahren, wenn im neuen Verfahren keine Einigung erzielt werden kann. Bei der frühen Nutzenbewertung nach $\S 35 a$ SGB V wird das IQWIG vom G-BA beauftragt, die vom Hersteller zur gewünschten Markteinführung verpflichtend vorzulegenden Dossiers wissenschaftlich zu bewerten. Der darin dargestellten Zusatznutzen hat eine unmittelbare Auswirkung auf die Preisfindungsverhandlungen beim G-BA (s. 5.2.8.1). In einer Pressemitteilung vom 11.07.2019 weist das Institut darauf hin, dass seit Einführen des Verfahrens bei rund der Hälfte der 216 analysierten Dossiers zur Neueinführung von Medikamenten keine Zusatznutzen nachgewiesen werden konnte, zumeist aufgrund methodischer Schwächen. Dieses Ergebnis wurde im BM] publiziert [109].

Durch das GKV-Versorgungsstrukturgesetz (GKV-VStG, s. 5.1.5) wurde im Jahr 2012 die „Erprobungsregel“ nach §137e SGB V eingeführt. Sie besagt, dass der G-BA neue Untersuchungs- und Behandlungsmethoden, die das „Potenzial einer erforderlichen Behandlungsalternative“ haben, durch eine Richtlinie zur Erprobung - verbunden mit klinischen Studien - zulassen kann, um die erforderlichen Erkenntnisse für eine Nutzenbewertung gewinnen zu können. Mit der Bewertung der Anträge der Hersteller auf dieses Verfahren wird das IGWIG beauftragt (sog. Potenzialbewertung).

Mit dem GKV-Versorgungsstärkungsgesetz (GKV-VSG, s. 5.1.8) wurde im Jahr 2015 durch den $\S 137 \mathrm{~h}$ SGB V die Bewertung von neuen Untersuchungs- und Behandlungsmethoden (NUB) für „Medizinprodukte hoher Risikoklassen“ eingeführt. Dies gilt für Methoden, die ein neues theoretisch-wissenschaftliches Konzept verfolgen, einen besonders invasiven Charakter haben und für die eine Vergütung nach $\S 6$ des Krankenhausentgeltgesetzes (NUB-Verfahren) beantragt wird. Auch in diesem Fall wird das IQWIG vom G-BA mit der Potenzialbewertung der Anträge der Hersteller und Krankenhäuser beauftragt.

Aus unserem Fachgebiet fallen z. B. die implantierbaren Hypoglossusstimulatoren bei Schlafapnoe unter das NUB-Verfahren.

Auch das Verfahren zum Vorschlagsrecht von Recherchethemen durch Einzelpersonen (sog. HTA-Verfahren) und die Beteiligung des IQWIG an internationalen wissenschaftlichen Organisationen wurde über das Gesetz geregelt.

\subsection{BÄK - Bundesärztekammer und LÄK - Landesärztekammern}

Die Bundesärztekammer (BÄK) ist keine Körperschaft des öffentlichen Rechts, sondern eine Arbeitsgemeinschaft der 17 Landesärz- 
tekammern, die ihrerseits jedoch Körperschaften des öffentlichen Rechts sind [123].

\subsubsection{Bundesärztekammer}

Die BÄK ist die Spitzenorganisation der ärztlichen Selbstverwaltung und vertritt als solche die berufspolitischen Interessen der Ärztinnen und Ärzte in Deutschland, wodurch sie Einfluss auf den gesundheitspolitischen Meinungsbildungsprozess der Gesellschaft ausübt. Der Deutsche Ärztetag ist die Jahreshauptversammlung der BÄK.

Die Aktivitäten der BÄK betreffen in vielen Aspekten die Qualität im Gesundheitswesen, die schwerpunktmäßig durch seinen „Ausschuss Qualitätssicherung“ und, gemeinsam mit den LÄK, die „Ständige Konferenz Qualitätssicherung“ koordiniert werden.

Als Stellungnahmeberechtigter beteiligt sich die BÄK intensiv an Verfahren, die beim G-BA liegen, oder kommentiert Papiere des IQTIG, des IQWIG oder Entwürfe des Gesetzgebers. Über die Arzneimittelkommission der Ärzteschaft (Akd̈̈) ist die BÄK in das frühe Nutzenbewertungsverfahren nach AMNOG eingebunden ( $\mathrm{s}$. 5.2.8.1) und ist Mitglied im Kuratorium des IQWIG. Weiterhin fördert die BÄK aktiv die Versorgungsforschung. Darüber hinaus unterhält die BÄK die „Datenbank ärztlicher Qualitätssicherungsinitiativen “ (ÄQSI, s. 6.10) und unterstützt dadurch freiwillige Qualitätsinitiativen, zu denen auch die Förderung des ärztlichen Peer-Review-Verfahrens zählt.

Durch spezialgesetzliche Regelungen hat die BÄK Richtlinienkompetenz beim Feststellen des aktuellen Standes der Erkenntnisse der medizinischen Wissenschaft auf dem Gebiet der Transplantationsmedizin (§16 TPG) und der Transfusionsmedizin (§18 TFG) und ist in die Bereiche Radiologie und Labormedizin involviert.

Ethische Richtlinien werden durch die Ethikkommission der BÄK, aber auch in der Musterberufsordnung [124], der die Genfer Deklaration (s. 2.2) vorangestellt ist, aufgestellt.

Die BÄK ist organisationsübergreifend beteiligt am „Ärztlichen Zentrum für Qualität in der Medizin“ (ÄZQ), am „Aktionsbündnis Patientensicherheit“ (APS [125]) und bis 2019 als Gesellschafter an der „Kooperation für Transparenz und Qualität im Gesundheitswesen GmbH“ (KTQ, s. 4.2). Als diesbezügliche Schwerpunkte werden „Patientensicherheit“, „Programm für Nationale Versorgungsleitlinien“ und das Curriculum „Fortbildung zur Qualitätssicherung“ genannt.

\subsection{2 ÄZQ - Ärztliches Zentrum für Qualität in der Medizin}

Das ÄZQ wird gemeinsam getragen von der BÄK und der Kassenärztlichen Bundesvereinigung (KBV). Es wurde bereits 1995 als „Zentralstelle der deutschen Ärzteschaft zur Qualitätssicherung in der Medizin“ gegründet und 2003 auf den aktuellen Namen umbenannt. Die Aufgabe des ÄZQ ist die Unterstützung von BÄK und KBV bei ihren Aufgaben im Bereich der Qualitätssicherung der ärztlichen Berufsausübung. Die im Leitbild festgelegten Ziele zur Qualitätsverbesserung im Gesundheitswesen sind:

- Qualitätssicherung/Qualitätsmanagement (QS/QM) bereichsübergreifend in allen Versorgungsbereichen gestalten

- QS/QM problemadäquat weiterentwickeln

- Prioritäten setzen

- Das Richtige tun: Leitlinien und Prinzipien der Evidenzbasierten Medizin in der Versorgung berücksichtigen

- Den Patienten einbeziehen
- Angemessene personelle und organisatorische Strukturen für QS/QM schaffen

- Professionalisierung auf dem Gebiet von QS/QM weiterentwickeln

- QS/QM in Kooperation aller Beteiligten weiterentwickeln

Die Grundsätze der Arbeitsweise des Instituts seien evidenzbasierte Medizin, Patientensicherheit, Patientenorientierung und Transparenz [126].

Das ÄZQ benennt 4 Aufgabenschwerpunkte: Medizinische Leitlinien, Patienteninformation, Patientensicherheit und Fehlervermeidung, und schließlich Qualitätsentwicklung in der Medizin. Darüber hinaus bietet das ÄZQ ein umfangreiches Schulungsprogramm an und ist international mit Partnerorganisationen verknüpft.

Im Bereich der Leitlinien wurde eine Zusammenarbeit mit der AWMF etabliert, um sog. „Nationale Versorgungsleitlinien“ (NVL) zu erstellen. Diese sollen als sektorenübergreifende Leitlinien zu Erkrankungen hoher Prävalenz die integrierte Versorgung unterstützen. Derzeit sind 7 NVL fertiggestellt für Asthma, COPD, KHK, Herzinsuffizienz, Kreuzschmerz, Depression, Diabetes, die achte für Hypertonie soll demnächst folgen [127]. Nach Aussage der BÄK verstehen sich die nationalen Versorgungsleitlinien als „ein allein ärztlichem Sachverstand und wissenschaftlicher Evidenz geschuldetes Konzept unter ausdrücklicher Einbeziehung der Bereiche der Prävention und der Rehabilitation“ in „Abgrenzung zu gesetzlichen Behandlungsprogramme nach § 137f SGB V (d. h. DMPs)“[128].

Weiterhin hat das ÄZQ in der gleichen Kooperation das Deutsche Leitlinien-Bewertungsinstrument (DELBI) entwickelt, das parallel zum international verwendeten AGREE II zum Clearing von Leitlinien eingesetzt werden kann. Mit der Gründung des IQWIG wurde das zuvor durchgeführte Leitstellenclearing an dieses übergeben.

Zur Patienteninformation wird die Website „Patienten-Information.de“ mit einem reichhaltigen Angebot an laienverständlichen Informationen vorgehalten.

Neben ausführlichen Informationen zur Patientensicherheit und zum Fehlermanagement betreibt das ÄZQ seit dem Jahr 2005 das „Netzwerk CIRSmedical.de“, das als Dachorganisation die größten und bedeutendsten fachspezifischen, überregionalen oder regionalen Fehlermeldesysteme (üFMS, s. 5.2.5.5) zusammenführt und derzeit 126 Kliniken vernetzt [129].

Schließlich bietet das ÄZQ noch ein umfangreiches Informations- und Fortbildungsprogramm für Qualitätsmanagementkonzepte an wie z. B. in der ambulanten Versorgung (Q-M-A).

\subsubsection{Landesärztekammern}

Die Landesärztekammern sind in erster Linie für die Qualitätssicherung der in Ihnen organisierten Ärzte zuständig. Dazu werden verbindliche Berufs-, Weiterbildungs- und Fortbildungsordnungen erstellt, die nicht nur das Verhalten als Arzt, sondern auch die fachliche Qualifikation regeln. Die Weiterbildungsordnungen werden in einem kontinuierlichen Prozess der Entwicklung des medizinischen Fortschritts und des ärztlichen Berufsstandes angepasst. Die ärztliche Fortbildung wird über die Vergabe von Fortbildungszertifikaten bei nachgewiesenem Besuch von zertifizierten Fortbildungs- 
veranstaltungen gewährleistet. Grundlage dafür sind bei Vertragsärzten § 95d und bei Krankenhausärzten § 136 b SGB V.

Darüber hinaus betreiben alle LÄK eigeninitiativ Qualitätsprojekte und sind in Gremien der externen Qualitätssicherung auf Landesebene eingebunden (z. B. LAG, s. 5.2.6.3; LQS, s. 5.2.6.4).

\subsection{AWMF - Arbeitsgemeinschaft der Wissenschaft- lichen Medizinischen Fachgesellschaften}

In der Arbeitsgemeinschaft der Wissenschaftlichen Medizinischen Fachgesellschaften e.V. (AWMF) sind 179 wissenschaftliche Fachgesellschaften (und 3 assoziierte) aus allen Bereichen der Medizin zusammengeschlossen. Die AWMF wurde 1962, initiiert von der Deutschen Gesellschaft für Chirurgie, mit zunächst 16 Mitgliedern in Frankfurt gegründet, um gemeinsame Interessen besser gegenüber dem Gesetzgeber und Organen der ärztlichen Selbstverwaltung vertreten zu können. Organe der AWMF sind die DelegiertenKonferenz und das Präsidium. Falls erforderlich, werden ad-hocKommissionen aus den Delegierten und ggf. Sachverständigen aus den Mitgliedsgesellschaften gebildet. Die AWMF hat 2 permanente interdisziplinäre Arbeitskreise (Ärzte und Juristen, Krankenhausund Praxishygiene) eingerichtet, betreibt das „AWMF-Institut für medizinisches Wissensmanagement (AWMF-IMWi)“ und gibt die Zeitschriften „German Medical Science“ (GMS), „Zeitschrift für Evidenz, Fortbildung und Qualität im Gesundheitswesen “ (ZEFQ, Mitherausgeber) und „Hygiene und Medizin“ (über den Arbeitskreis Krankenhaus- und Praxishygiene) heraus.

Die AWMF beschreibt ihre Ziele folgendermaßen:

„Die AWMF berät über grundsätzliche und fachübergreifende Fragestellungen in der wissenschaftlichen Medizin, fördert die Zusammenarbeit ihrer Mitgliedsgesellschaften bei der Wahrnehmung ihrer wissenschaftlich-medizinischen Aufgaben und Ziele sowie den Transfer wissenschaftlicher Erkenntnisse in die ärztliche Praxis. Darüber hinaus vertritt sie in Kooperation mit anderen ärztlichen Organisationen die Interessen der wissenschaftlichen Medizin gegenüber den zuständigen politischen Gremien und der Öffentlichkeit, strebt eine enge Zusammenarbeit mit vergleichbaren Organisationen an und stellt somit einen wichtigen Pfeiler in der medizinischen Organisation Deutschlands dar" [130].

Wichtige Aktivitäten betreffen:

- Herausgabe der ärztlichen Leitlinien der medizinischen Fachgesellschaften

- Mitarbeit an den Nationalen Versorgungsleitlinien

- Mitarbeit am Leitlinienprogramm Onkologie

- Aus-, Weiter- und Fortbildung in der Medizin

- Interdisziplinäre Zusammenarbeit der Fächer

- Mitarbeit an den Klassifikationssysteme in der Medizin (z. B. ICD, OPS)

- Zugänglichkeit von wissenschaftlicher Literatur

Die AWMF ist an bestimmten Gremien der Selbstverwaltung im Gesundheitswesen direkt beteiligt (z. B. Kuratorium des IQWIG) und zur Stellungnahme berechtigt $u$. a. bei den Methoden und der Entwicklung von Qualitätsindikatoren durch das IQTIG und bei Disease-Management-Programmen gegenüber dem G-BA. Kooperationen bestehen über das ÄZQ mit der BÄK und KBV (s. 5.5.2), mit der Deut- schen Krebsgesellschaft und der Deutschen Krebshilfe und mit dem „Deutschen Netzwerk für Versorgungsforschung“ (DNVF).

Zum Qualitätsmanagement nimmt die AWMF folgendermaßen Stellung:

„Hochwertige Leitlinien und die aus ihnen abgeleiteten Indikatoren für die Prozessqualität, Befragungen von Bürgern, Patienten, Mitarbeitern und externen Mitversorgern, lesbare Qualitätsdarlegungen, medizinisch orientierte Zertifizierungen und BenchmarkTechniken („Lernen von den Guten“) sind neben anderen Instrumente des Qualitätsmanagements, die von den wissenschaftlichen medizinischen Fachgesellschaften mitentwickelt, gelehrt und eingesetzt werden. Weitere wichtige Aufgaben sieht die AWMF in der Umsetzung und Evaluierung des medizinischen Qualitätsmanagements“ [131].

Die AWMF ist also wesentlich mehr als die Institution, die die fachlichen und organisatorischen Rahmenbedingungen für die medizinischen Leitlinien bereitstellt, auch wenn das eine sehr wichtige Aufgabe zum Sichern einer fachgebietsspezifischen oder -übergreifenden Behandlungsqualität ist: Sie ist der konzentrierte Sachverstand der deutschen Ärzteschaft. Sie ist ein wichtiger integrierender Akteur, der die Kompetenz der Mitgliedsgesellschaften für wissenschaftliche und versorgungspolitische Fragestellungen - und damit auch für die Qualität im Gesundheitswesen - einsetzt. Die AWMF hat eine gewichtige, kritische Stimme in der Gesundheitspolitik und bestimmt durch ihre Stellungnahmen, zu denen sie z. T. gesetzlich legitimiert ist, die politische und fachliche Entwicklung des Gesundheitswesens mit.

\subsection{KBV - Kassenärztliche Bundesvereinigung und KV - Kassenärztliche Vereinigungen}

Die Kassenärztliche Bundesvereinigung (KBV) ist eine Körperschaft des öffentlichen Rechts und der Dachverband der 17 Kassenärztlichen Vereinigungen (KV). Ihre Aufgaben umfassen 3 Bereiche: Interessenvertretung der derzeit rund 172000 freiberuflichen Ärzte und Psychotherapeuten gegenüber den gesetzlichen Krankenkassen und in gesundheitspolitischen Belangen, Sicherstellung der ambulanten ärztlichen Versorgung für die Versicherten und Mitarbeit an der Versorgungsplanung und -gestaltung.

KBV und auf Landesebene KVen sind fest verankert im System der gesetzlich vorgeschriebenen Qualitätssicherung und nehmen zusammen mit Vertretern der Deutschen Krankenhausgesellschaft (DKG) die Interessen der sog. Leistungserbringer wahr. Sie besetzen Positionen in Gremien des G-BA, IQTIG, IQWIG und der LAG. Durch die Möglichkeit, in diesen Gremien mitzuentscheiden, haben sie große Gestaltungsmöglichkeiten auch und gerade für Qualitätsaspekte in der Gesundheitspolitik.

Neben der „Berichterstattung über den Umsetzungsstand des Qualitätsmanagements in Vertragsarztpraxen, Vertragspsychotherapeutenpraxen und Medizinischen Versorgungszentren (MVZ)“ gemäß der Qualitätsmanagement-Richtlinie des G-BA (QM-RL, s. 5.2.5.1, 5.2.6.2) stellt das Durchführen der Qualitätssicherungsmaßnahmen nach §135 Abs. 2, §135 Abs. 1 und §135b Abs. 2 SGB V die zentrale Aufgabe der KVen im Bereich Qualitätssicherung und Patientenorientierung dar.

Aufgrund dieser Regelungen sind eine kontinuierlich wachsende Anzahl von Leistungsbereichen unter Genehmigungsvorbehalt 
gestellt worden. Im Jahr 2018 waren das insgesamt 52 Leistungsbereiche, davon 5 Disease-Management-Programme. Unser Fachgebiet betreffen die Leistungsbereiche „Hörgeräteversorgung“ und „Hörgeräteversorgung (Kinder)“. Bei Antrag auf Genehmigungserteilung prüft die KV mit Unterstützung der bei ihr eingerichteten Qualitätssicherungskommission, ob die Genehmigungsvoraussetzungen sowohl in Bezug auf die fachliche Befähigung des Antragstellers als auch auf weitere Merkmale der Strukturqualität seiner Praxis gegeben sind. Laut „Qualitätsbericht 2018“ wurden im Jahr 2017 unter Mitwirkung von 3199 Mitgliedern der Qualitätskommissionen „283218 Genehmigungen zu verschiedenen Leistungsbereichen, zusätzlich 38357 Genehmigungen für Psychotherapie erteilt“. Anschließend wird das Fortbestehen der Voraussetzungen für die Genehmigung durch verschiedene Verfahren geprüft. Als Ergebnis dieser Überprüfungen wurde „In insgesamt 410 Fällen ... die Genehmigung widerrufen“ [132].

Weiterhin unterstützen und fördern die KVen praxisübergreifende Qualitätszirkel, die in den Qualitätssicherungsrichtlinien der KBV als anerkanntes Qualitätsinstrument beschrieben sind. Durch die Teilnahme an zertifizierten Qualitätszirkeln können Fortbildungspunkte erworben werden.

Schließlich bieten die KVen ein Qualitätsmanagementsystem für Praxen und MVZ an, das „QEP - Qualität und Entwicklung in Praxen“(s. 4.6).

\subsection{DKG - Deutsche Krankenhausgesellschaft und LKG - Landeskrankenhausgesellschaften}

Die Deutsche Krankenhausgesellschaft (DKG) e.V. ist der Dachverband der Krankenhausträger, d. h. von insgesamt 28 Mitgliedsverbänden, von denen 16 Landeskrankenhausgesellschaften sind und 12 Spitzenverbände wie z. B. der „Verband der Universitätsklinika Deutschlands“ (VUD) [133].

Die DKG vertritt die Interessen ihrer Mitgliedsverbände im System der gesetzlich vorgeschriebenen Qualitätssicherung und nimmt zusammen mit Vertretern der Kassenärztlichen Bundesvereinigung (KBV) die Interessen der sog. Leistungserbringer wahr. Sie besetzt Positionen in Gremien des G-BA, IQTIG, IQWIG und der LAG. Durch die Möglichkeit, in diesen Gremien mitzuentscheiden, hat sie große Gestaltungsmöglichkeiten auch und gerade für Qualitätsaspekte in der Gesundheitspolitik.

Den Landeskrankenhausgesellschaften sind die Landesgeschäftsstellen für Qualitätssicherung (LQS, [95]) zugeordnet, die bei der stationären Qualitätssicherung nach QSKH-RL eine zentrale Rolle spielen (s. 5.2.6.4).

Weiterhin betreibt die DKG eine aktive Öffentlichkeitsarbeit, um die Positionen ihrer Mitglieder als „Stimme der Krankenhäuser“ zu aktuellen gesundheitspolitischen Diskussionen darzustellen. Im aktuellen Positionspapier „Patientenwohl und Daseinsvorsorge“ werden u. a. folgende Punkte hervorgehoben: [134].

1. Qualität stärken, Transparenz herstellen

2. Personal fördern, Fachkräftemangel begegnen

3. Mehr Zeit für den Patienten, Bürokratie abbauen

4. Investitionen nachhaltig finanzieren, moderne Strukturen ermöglichen

5. Digitalisierung beschleunigen, E-Health ausbauen

6. Innovationen stärken, medizinischen Fortschritt gewährleisten

\subsection{Spitzenverband-GKV und Landesverbände der Krankenkassen}

Der Spitzenverband-GKV ist eine Körperschaft des öffentlichen Rechts, deren Mitglieder alle gesetzlichen Krankenkassen sind [135]. Er versteht sich als die zentrale Interessenvertretung aller Kranken- und Pflegekassen. Organisatorisch besteht er aus Mitgliederversammlung, Verwaltungsrat und Vorstand, dem ein Fachbeirat zugeordnet ist. Das Ziel des Spitzenverband-GKV ist nach eigener Aussage „die Rahmenbedingungen für einen intensiven Wettbewerb um Qualität und Wirtschaftlichkeit in der gesundheitlichen und pflegerischen Versorgung“ zu gestalten.

Der Spitzenverband-GKV bzw. die Landesverbände der Krankenkassen sind im System der gesetzlich vorgeschriebenen Qualitätssicherung als Kostenträger das Gegengewicht zu den sog. Leistungserbringern. Sie entsenden üblicherweise eine zu den Leistungserbringern paritätische Anzahl von Vertretern in die Gremien des G-BA, IQTIG, IQWIG und der LAG bzw. LQS. Durch die Möglichkeit, in diesen Gremien mitzuentscheiden, haben sie erheblichen Einfluss auf die Ausgestaltung der Gesundheitspolitik inkl. ihrer Qualitätsdimensionen.

Zur Qualitätssicherung im Bereich der Krankenversicherung nennt der Spitzenverband-GKV 6 Bereiche: die Mindestmengenregelung (s. 5.2.5.4), die Qualitätsberichte der Krankenhäuser (s. 5.2.6.1), die finanzielle Förderung klinischer Krebsregister, die einrichtungsübergreifende Qualitätssicherung bei Vorsorge- und Rehaeinrichtungen, ärztliche Arbeitsprozesse und G-BA-Richtlinien für den vertragsärztlichen Bereich (s. 5.7, KV-basierte ambulante Qualitätssicherung) sowie eine Studie über die Ergebnisqualität von Hebammen und Geburtshäusern.

Schließlich betreibt der Spitzenverband-GKV eine aktive Öffentlichkeitsarbeit, um als „Sachwalter der Beitragszahler“ zu aktuellen gesundheitspolitischen Themen Stellung zu beziehen.

\subsection{MDK - Medizinischer Dienst der Krankenversi- cherung}

Der Medizinische Dienst der Krankenversicherung (MDK) ist der sozialmedizinische und pflegefachliche Beratungs- und Begutachtungsdienst für die gesetzliche Kranken- und Pflegeversicherung [136]. Es gibt 15 teils bundeslandübergreifende MDKs und einen „Medizinische Dienst des Spitzenverbandes Bund der Krankenkassen“ (MDS), der die Arbeit der MDKs koordiniert und z. B. über Richtlinien für einheitliche Begutachtung unterstützt [137]. MDKs und MDS bilden zusammen die sog. MDK-Gemeinschaft.

Insgesamt 4 Kompetenz-Zentren unterstützen die MDK-Gemeinschaft zu den Themen Geriatrie, Onkologie, Psychiatrie und Psychotherapie sowie Qualitätssicherung und Qualitätsmanagement (KCQ). Letztgenanntes Kompetenz-Zentrum ist beim MDK Baden-Württemberg angesiedelt. Es sieht seine Rolle vorrangig in der wissenschaftlichen Systemberatung der MDK-Gemeinschaft [138]. Weiterhin unterstützen 6 sozialmedizinische Expertengruppen (SEG) zu Fragen der bundesweit einheitlichen Begutachtung die MDK-Gemeinschaft.

Der MDK wird auf Grundlage des § 281 Abs. 1 SGB V durch eine Umlage der Kranken- und Pflegekassen finanziert.

Seine Mission ist sicherzustellen, dass die Leistungen der Kranken- und der Pflegeversicherung nach objektiven medizinischen Kriterien allen Versicherten zu gleichen Bedingungen zugutekommen. Entsprechend der grundsätzlichen Regelung in den Paragra- 
fen $§ 2, \S 12$ und $\S 70$ des SGB V, d. h. dem Wirtschaftlichkeitsgebot und dem Anspruch auf eine dem allgemein anerkannten Stand der medizinischen Erkenntnisse entsprechende Leistung (s. 5.1), entscheidet der MDK, welche Leistungen notwendig zur Versorgung der Versicherten sind.

In seiner Publikation „Zahlen, Daten, Fakten 2018“ berichtet der MDS/MDK für das Berichtsjahr 2018 über insgesamt 5729000 sozialmedizinischen Empfehlungen für die Krankenkassen, davon als größte Position 2580000 Krankenhausabrechnungsprüfungen, was einer Prüfquote von ca. 17 \% entspricht. Der Umfang dieser Abrechnungsprüfungen und der damit verbundene erhebliche Aufwand auf Krankenhausseite ist Gegenstand intensiver gesundheitspolitischer Diskussionen. Die geringste Anzahl von Empfehlungen erfolgte mit 14000 zur Position „Behandlungsfehler“ [139].

Aufgrund der MDK-QK-RL des G-BA hat der MDK weiterhin im Rahmen der externen Qualitätssicherung von Krankenhäusern die Aufgabe, nach Beauftragung Vor-Ort-Kontrollen durchzuführen (s. 5.2.7.1).

Aktuell wird vom BMG eine Gesetzgebung vorbereitet, die eine drastische Neukonfiguration des MDK vorsieht (MDK-Reformgesetz).

\subsection{SVR -Sachverständigenrat Gesundheit}

Der „Sachverständigenrat zur Begutachtung der Entwicklung im Gesundheitswesen“, der auf Grundlage des $§ 142$ SGB V besteht und dessen Mitglieder vom Bundesgesundheitsminister auf 4 Jahre berufen werden, hat die Aufgabe, alle 2 Jahre ein Gutachten vorzulegen, um

- „die Entwicklung in der gesundheitlichen Versorgung mit ihren medizinischen und wirtschaftlichen Auswirkungen zu analysieren,

- unter Berücksichtigung der finanziellen Rahmenbedingungen und vorhandenen Wirtschaftlichkeitsreserven Prioritäten für den Abbau von Versorgungsdefiziten und bestehenden Überversorgungen zu entwickeln,

- Vorschläge für medizinische und ökonomische Orientierungsdaten vorzulegen, sowie

- Möglichkeiten und Wege zur Weiterentwicklung des Gesundheitswesens aufzuzeigen “ [140].

Der SRV Gesundheit vertritt in seinem aktuellen Gutachten und in Stellungnahmen und Pressemitteilungen die Auffassung, dass eine deutliche Umstrukturierung der Krankenhauslandschaft notwendig sei, die derzeit durch Über- und Unterversorgung gekennzeichnet sei $[141,142]$ (siehe auch 8.1.6).

\subsection{Diskussion - wie Qualität messen?}

In den letzten 15 Jahren ist vom Gesetzgeber und dem G-BA mit seinen Instituten ein zunehmend differenziertes, komplexes System der externen Qualitätssicherung entwickelt worden. Die ärztlichen Institutionen der gemeinsamen Selbstverwaltung tragen durch Mitarbeit in den Gremien zur externen Qualitätssicherung bei. Unabhängig davon haben sie weitere wichtige Aufgaben übernommen, deren Anfänge teilweise wie z. B. in der Transfusionsmedizin deutlich vor den Beginn der gesetzlichen Qualitätssicherung zurückreichen.

Die externe Qualitätssicherung beruht überwiegend auf der Auswertung von Qualitätsindikatoren, die sich auf die Prozessqua- lität beziehen, und zu einem geringen Anteil auf Vorgaben für die Strukturqualität (Ausstattung und Qualifikation). Diese beiden Qualitätsdimensionen lassen sich leichter messen als die Ergebnisqualität, die sich oft nur sektorenübergreifend erfassen lässt und neben objektiven auch subjektiv empfundene Elemente enthält.

\subsubsection{Qualitätsindikatoren in der externen stationären Qualitätssicherung (nach QSKH-RL)}

Zur gesetzlichen externen Qualitätssicherung stellt die AWMF fest, dass sie inklusive der verwendeten Qualitätsindikatoren lückenhaft sei, da sie nur auf kleine Teilbereiche bezogen sei. Von daher könne sie keine Aussage über das ganze Gesundheitssystem machen. Es wird gefordert, sie auf alle Krankenhausbereiche auszudehnen [143]. Weiterhin kritisiert die AWMF in einer Stellungnahme zum Grundsatzpapier des IQTIG, dass zur Beurteilung der Qualität immer noch ausschließlich das „Tracer-Konzept“ ( = Qualitätsindikatoren) herangezogen werde. Es gebe aber keine Evidenz dafür, dass dadurch auf die Qualität einer Gesamtorganisation geschlossen werden könne. Vielmehr sei neben der Betrachtung der „Mikroebene“ der direkten Leistungserbringung (durch Qualitätsindikatoren) auch die „Mesoebene“ (das Krankenhaus) und die „Makroebene“ (das Gesundheitssystem) mit einzubeziehen. Zur Mesoebene gehörten einrichtungsbezogene Anforderungen wie Führung, Mitarbeiterorientierung und internes Qualitätsmanagement, zur Makroebene z. B. die Gewährleistung der Versorgungsgerechtigkeit. Bleibe es allerdings bei dem vom IQTIG verfolgten Tracer-Konzept und werde der Blick nicht mit auf die einrichtungsinternen Strukturen und das Qualitätsmanagement der Patienten versorgenden Einrichtungen gelegt, könne eine Organisationskultur, die an Patientenzentrierung ausgerichtet sei, nicht gelingen [102]. In der Essenz fordert die AWMF damit, die gesetzliche Qualitätssicherung in Richtung eines Akkreditierungssystems auszubauen (!).

Die BÄK schließt sich der Kritik an. Die in der externen Qualitätssicherung verwendeten Qualitätsindikatoren seien oft nicht in der Lage, echte Qualitätsmängel zu identifizieren. Deshalb würden vom IQTIG mit Patientenbefragung, Verwendung von Abrechnungsdaten und stichprobenhaften Einrichtungsbegehungen neue Instrumente entwickelt. Sinnvoll sei die Erfassung von Langzeitergebnisse über die Sektorengrenzen hinweg [144]. Auch die Deutsche Gesellschaft für Innere Medizin (DGIM) fordert das. Sie erwartet sich vom bevorzugten Einsatz von Ergebnisparametern positive Steuerungseffekte auf die Leistungserbringer [145].

\subsubsection{Qualitätsindikatoren für die Krankenhausplanung (planQI-RL)}

Sowohl BÄK als auch AWMF kritisieren den Ansatz des IQTIG als unzureichend, ausschließlich Qualitätsindikatoren aus der externen stationären Qualitätssicherung zu verwenden. Andere Anforderungen wie abteilungsübergreifende Verbesserung der Patientensicherheit, wohnortnahe Sicherstellung der Basisversorgung und ausreichende Ausstattung mit qualifiziertem Personal seien wesentliche, zu berücksichtigende Aspekte. Bei zunehmender Ambulantisierung und sektorenübergreifender Versorgungsplanung müsse auch die Qualitätssicherung sektorenübergreifend sein. Durch das reine Erfassen von Struktur-, Prozess- und ggf. Ergebnisqualität sei kein ausreichender Steuerungseffekt zu erreichen; 
planQI-RL sei deshalb kein umfassendes, tragfähiges Konzept für eine qualitätsorientierte Krankenhausplanung [144, 145] (s. auch 8.1.6). Acht Bundesländer sehen das offenbar auch so und haben beschlossen, diese Methode nicht für ihre Krankenhausplanung zu verwenden (s. 5.2.6.8).

\subsubsection{Qualitätsindikatoren für qualitätsabhängige Vergütung}

Für die Wirksamkeit von qualitätsabhängigen Zu- oder Abschlägen der Vergütung, dem sog. „Pay for Performance“ (P4P) konnten Untersuchungen in anderen Gesundheitssystemen keinen positiven Zusammenhang zwischen Bonuszahlungen und Ergebnissen finden [146-149]. Dagegen bestehen durch P4P hohe Anreize zur Patientenselektion bzw. Patientenfehlsteuerung [144] .

\subsubsection{Mindestmengenregelung (Mm-R)}

Mindestmengen für $\mathrm{Cl}$ wurden auch in der HNO-Heilkunde diskutiert. Sie sind generell innerärztlich höchst umstritten. Es sollte vor Einführung eine klare Evidenz für mengenbezogene Qualitätsunterschiede vorliegen (z. B. $[150,151])$. Problematisch seien nach Meinung der BÄK insbesondere die Gleichsetzung einer Mindestmenge mit Qualität sowie der Fehlanreiz des Erreichens einer Schwelle durch ein Krankenhaus vor dem Hintergrund ärztlicher Indikationsstellung [144]. Von Seiten des BGM besteht die Absicht, Mindestmengen auszudehnen und als Instrument der Strukturpolitik zu verwenden [152].

\section{Einrichtungsinternes und -übergreifendes Qualitätsmanagement}

Alle vorgenannten verpflichtenden gesetzlichen und institutionellen Regulierungen und freiwilligen Zertifizierungs-, Akkreditierungs- und Exzellenzsysteme können nicht direkt Qualität in den Gesundheitsorganisationen erzeugen; sie können nur unterstützend oder fordernd auf die Akteure in der unmittelbaren Krankenversorgung einwirken. Das tatsächliche Qualitätsniveau im Behandlungsprozess entsteht täglich neu als Resultat der gemeinsamen Anstrengung von Ärzten und Pflegenden, Leitungen, indirekt an der Krankenversorgung Beteiligten, internen und externen Partnern und der Administration. Im Folgenden werden die Bereiche aufgeführt, die einen relevanten Einfluss darauf haben.

\subsection{Führung}

Die Führung steht nicht nur vor der Herausforderung, bei begrenzten personellen und materiellen Ressourcen funktionierende Abläufe verantwortlich zu organisieren, sondern in einem sich stetig ändernden Umfeld die Organisation immer wieder neu einzustellen und auszurichten.

Der Beitrag der Führung zum Qualitätsmanagement ist erheblich. Neben dem Vorleben einer integren Haltung und der Fähigkeit zur Inspiration der Mitarbeiter können ohne die Top-Down-Unterstützung der Führung Maßnahmen zum Qualitätsmanagement weder eingeführt noch nachhaltig aufrechterhalten werden. Von der Einführung eines vollständigen Qualitätsmanagementsystems profitiert sie selbst außerordentlich, da sich aus dessen Anwendung wertvolle Hinweise für Verbesserungsbereiche, die Entwicklung der Gesamtorganisation und eine ggf. notwendige Neuausrichtung ergeben.

\subsection{Qualitätsmanagementsystem}

Die Funktion eines Qualitätsmanagementsystems wurde in vorangegangenen Kapiteln bereits ausführlich beschrieben (s. 4.2, 4.3, 4.4 und 5.2.5.1).

Sie liegt v. a. darin, alle Aspekte des Aufrechterhaltens und Verbesserns der Qualität einer Organisation in einer systematischen Weise zu unterstützen.

\subsection{Ziele, Ergebnisse und Entwicklung}

Ohne das Definieren von Zielen, das Messen der Zielerreichung im Intervall und ggf. die Implementation von Korrekturen, d. h. die Anwendung des PDCA-Zyklus, ist die zielgerichtete Entwicklung einer Organisation erheblich erschwert. Ein hoher Umsetzungsgrad dieser Methode findet sich bei den ökonomischen bzw. betriebswirtschaftlichen Zielen einer Organisation, ausgedrückt z. B. in Casemix-Punkten und Budgeteinhaltung. Bei den medizinischen und patientenbezogenen Zielen sind Umsetzungs- und Durchdringungsgrad der PDCA-Methode wesentlich heterogener und stark davon abhängig, ob ein Qualitätsmanagementsystem eingesetzt wird. Sie ist dort jedoch nicht weniger hilfreich als beim Management der ökonomischen Parameter.

\subsection{Forschung und Innovation}

Den menschlichen Geist zeichnet die Fähigkeit zum Erkennen aus, es ist in der Tat eine seiner Kerneigenschaften. Die Neugier, die Welt zu verstehen, ist der Antrieb für Forschung, Kreativität und Innovation.

In der Medizin kommen akademische Forschung, klinische Innovationen und technologischer Fortschritt der Industrie auch und gerade in ihrem Zusammenwirken unmittelbar der medizinischen Versorgungsqualität und damit den Patienten zu Gute.

Die hohe Innovationsrate des medizinischen Wissens und damit seine verkürzte Gültigkeitsdauer sowie die permanente Zunahme seines Volumens machen ein aktives Wissensmanagement unabdingbar. Das schließt den Wissenstransfer an die nachfolgende Generation in studentischer Lehre und Weiterbildung sowie das Aktualisieren des Wissenstandes durch Fortbildungen und seine Anwendung in der klinischen Praxis mit ein.

\subsection{Prozesse}

\subsubsection{Standardisierung von Prozessen}

Die Standardisierung ist ein probates Mittel der Fehlervermeidung und kommt deshalb besonders in Bereichen zur Anwendung, in denen Fehler gravierende Folgen haben können (z. B. in der Luftfahrt). Prozesse lassen sich dann gut standardisieren, wenn sie prinzipiell immer gleich ablaufen, sodass durch die Standardisierung unerwünschte Abweichungen von einem idealen Prozessablauf vermieden werden können:

- Prozesse, die von Risikomanagement erfasst werden

- Dazu gehört z. B. das Time-Out vor Operationen, um Patienten-, Eingriffs- und Seitenverwechselungen zu vermeiden.

- Behandlungsprozesse

- Durch SOPs (Standard Operating Procedures), Pflegestandards, Behandlungspfade, Anwendung von Leitlinien und, 
wenn vorhanden, Erkenntnissen der EBM (Evidence Based Medicine) soll eine konsistente, dem aktuellen Stand der medizinischen Erkenntnis entsprechende Behandlungsqualität sichergestellt werden.

- Schriftliche Anweisungen

- Arbeits-, Verfahrensanweisungen und Qualitätshandbücher beschreiben mit unterschiedlicher Detailliertheit Prozesse und legen deren Ablauf verbindlich fest.

\subsubsection{Prozessoptimierung}

Prozesse können auf unterschiedliche Weise verbessert werden, sei es durch ad-hoc-Qualitätszirkel und -projekte, die ggf. durch interne oder externe Berater unterstützt werden, permanente Qualitätszirkel, oder im Rahmen der Anwendung eines Qualitätsmanagementsystems.

\subsection{Risiko- und Fehlermanagement}

\subsubsection{Administratives Risikomanagement}

Um vermeidbaren Schaden von Patienten, aber auch von Mitarbeitern abzuwenden, werden aufgrund gesetzlicher Vorschriften Vorkehrungen und Regelungen für Notfälle, Hygiene, Arzneimittelsicherheit sowie Informations- und Datensicherheit getroffen. Weiterhin werden Verantwortliche benannt und regelmäßige Schulungen durchgeführt.

\subsubsection{Fehlermanagement}

Das Fehlermanagement ist ein Teil des Risikomanagements. Mit verschiedenen Methoden wird das Prinzip verfolgt, über klinische Erfahrungen zu reflektieren und Begebenheiten, die potenziell einen Schaden für einen Patienten hätten verursachen können oder ihn tatsächlich verursacht haben, transparent darzustellen. Dadurch soll nicht nur die Achtsamkeit für individuelle Ursachen von Fehlern geschärft werden, sondern auch ggf. mitverantwortliche organisatorische oder technische Ursachen identifiziert werden.

Ein grundlegender Beitrag zur Fehlertheorie, insbesondere in Bezug auf die Medizin, wurde von James Reason geleistet. Er unterscheidet zunächst zwischen aktiven Fehlern und vorbestehenden latenten Konditionen. Während aktive Fehler sofort manifest werden, kann es bei latenten Konditionen Jahre dauern, bis sie eine Auswirkung zeitigen. Deshalb ist ihre Mitbeteiligung weniger offensichtlich. Aktive Fehler beruhen z. B. auf Unaufmerksamkeit, Uninformiertheit, Nicht-Wissen oder bewusstem Abweichen vom üblichen Procedere. Latente Konditionen können fehler-provozierende Arbeitsbedingungen sein wie Zeitdruck, Unterbesetzung, ungenügende technische Ausstattung usw., oder dauerhafte Schwachstellen wie schlecht gestaltete Arbeitsabläufe und räumliche Gegebenheiten oder Unzuverlässigkeit von technischen Geräten und Material.

Manifest werdende (Beinahe-) Fehler lassen sich in aller Regel auf eine Abfolge des Versagens verschiedener Sicherheitsmaßnahmen einschließlich der abschließenden aktiven Fehlhandlung zurückführen. Reason hat das anschaulich mit seinem „SchweizerKäsescheiben-Modell“ beschrieben: Jede Käsescheibe stellt eine Sicherheitsebene dar, inkl. der letzthandelnden Person. Die Löcher in den Käsescheiben variieren dynamisch in ihrer Größe und Position. Nur wenn ein Unglücksstrahl (d. h. das Potenzial für ein schädliches Ereignis) durch alle Käsescheibenebenen passieren kann, weil die Löcher sich zufällig überlagern, kommt er zum Tragen. Im Normalfall wird das Schadenspotenzial bei Versagen einer Ebene durch die nächste Ebene abgefangen.

Er differenziert 2 Herangehensweisen an menschliche Fehler:

- Personenbezogene Herangehensweise

- Sie ist die traditionelle Herangehensweise und fokussiert sich nur auf die hinter dem aktiven Fehler stehende Person. Sie reagiert mit (Nach-) Schulung, Schuldzuweisung („naming, blaming, shaming“) und ggf. disziplinarischen Maßnahmen. Beinahe-Fehler werden in dieser Kultur überwiegend nicht berichtet werden. Das Potenzial, aus ihnen zu lernen, wird verschenkt.

- Systembezogene Herangehensweise

- Sie versteht, dass Menschen nicht unfehlbar sind und deshalb aktive Fehler erwartet werden müssen. Sie gestaltet eine Kultur des Vertrauens, um einen möglichst vollständigen Fluss von Berichten über unerwünschte Ereignisse zu ermöglichen. Die Analyse dieser Berichte erlaubt die Identifikation von latenten Konditionen, die im Gegensatz zur menschlichen Fehlerneigung dauerhaft und nachhaltig korrigiert werden können [4, 5, 153].

Im Folgenden finden sich Anwendungen der systembezogenen Herangehensweise:

- Fehlerkonferenzen

- Üblicherweise anonymisierte Darstellung von (Beinahe-) Fehlern im Behandlungsprozess im Rahmen einer Teambesprechung

- M\&M-Konferenzen

- Morbiditäts- und Mortalitätskonferenzen kommen in der HNO-Heilkunde wegen der im Vergleich zu anderen Disziplinen deutlich geringeren Krankheitsschwere der Patienten kaum zum Einsatz

- Fehlermeldesysteme

- Fehlermeldesysteme (FMS), auch als CIRS (Critical Incident Reporting System) bekannt, erlauben es jedem Mitarbeiter, anonym und zeitnah bei einer zentralen Stelle eine Begebenheit mit dem Potenzial zur Patientenschädigung zu melden. Neben den einrichtungsinternen FMS gibt es auch einrichtungsübergreifende FMS (s. 6.10.2, 5.2.5.5). Die Meldungen werden zentral ausgewertet, auf mögliche Handlungsrelevanz geprüft und abschließend öffentlich im System kommentiert.

\subsubsection{Opus primum und Assistenzsysteme - Klinische Methoden des Risikomanagements}

Insbesondere in den chirurgischen Fächern oder solchen mit invasiven Prozeduren müssen manuelle Fertigkeiten und operative Strategien erlernt werden, deren Aneignung einer Lernkurve folgt. Eine Supervision durch einen Erfahrenen kann diesen Effekt abmildern, aber nicht aufheben. Der Grad der Zielerreichung des Eingriffs und des Vermeidens von Kollateralschäden werden sich bei einem Lernenden von dem eines Erfahrenen meist unterscheiden, so groß die Ernsthaftigkeit und Vorsicht des Lernenden auch sein mag - mit häufig geringen, je nach Art des Eingriffs aber auch schwerwiegenden Folgen [154, 155]. Die ethische Rechtfertigung dafür ist, dass unsere Lebensspanne und damit auch unsere Tätigkeitsdauer im 
ärztlichen Beruf begrenzt sind. Würde nicht eine permanente Ausbildung von Nachwuchs erfolgen, würde die Qualität der medizinischen Versorgung in kurzer Zeit degenerieren.

Folgende Methoden kommen in der chirurgischen Ausbildung zur Qualitätssicherung zum Einsatz:

- Formatives Prüfungsgespräch (sog. „Vorsingen“) vor dem Ersteingriff, um sich des konzeptionellen Verständnisses des Eingriffs und der Kenntnisse der chirurgischen Anatomie zu vergewissern

- Simulationsmodelle bzw. Phantommodelle, in der HNO z.B. für das Felsenbein erhältlich, ggf. in ein Surgical Skills Labs eingebettet

- Operationskurse mit Leichenpräparaten, Präparationsübungen an explantierten menschlichen Felsenbeinen

- Anders als noch vor 2 Jahrzehnten ist der Zugang zu Körpern von Verstorbenen in der Pathologie, an denen ohne äußerlich sichtbare Mutilation Operationsschritte geübt werden konnten, durch die aktuelle Rechtslage praktisch verschlossen

- Intraoperative Assistenz und Supervision durch einen Facharzt oder Oberarzt, bis der sog. Facharztstandard erreicht ist

Über die Ausbildungssituation hinaus werden generell Methoden angewandt, die nicht gesetzlich eingefordert werden, sondern "gute klinische Praxis“ sind:

- Chirurgische Assistenzsysteme wie das Neuromonitoring und Navigationssysteme unterstützen den Chirurgen darin, gefährdete wichtige anatomische Strukturen des Patienten zu schonen, aber auch in schwierigen anatomischen Verhältnissen das Operationsziel zu erreichen.

- Klinische Nachkontrollen erlauben zumindest teilweise die Beurteilung der Ergebnisqualität und stellen wertvolles Feedback für den Chirurgen dar.

\subsubsection{Ethikkommissionen}

Ethikkommissionen sind eine weitere Instanz, um Schaden von Patienten abzuwenden. Ihre Tätigkeit erfolgt auf Grundlage der Deklaration von Helsinki (s. 2.3).

\subsection{Patientenorientierung}

Eine klare Patientenorientierung entspricht nicht nur der ärztlichen Ethik, sondern ist auch eine prominente Anforderung aller gesetzlichen Regulierungen (z. B. 5.1.6) und freiwilligen Akkreditierungund Zertifizierungssysteme, weil sie eine unabdingbare Voraussetzung für das Erreichen guter Qualität ist.

\subsubsection{Patienteninformation und -aufklärung}

Es gehört mittlerweile zur guten Praxis, Patienten in laienverständlicher Sprache über Krankheitsbilder, die behandelnde Institution und Behandlungsabläufe zu informieren. Die Aufklärung der Patienten zur Einwilligung in invasive Prozeduren hat einen hohen Standard erreicht, nicht zuletzt aufgrund der rigiden Rechtsprechung der letzten Jahre.

\subsubsection{Partizipative Entscheidungsfindung}

Die sog. „partizipative Entscheidungsfindung“ basiert auf der internationalen „shared decision making“ (SDM) - Initiative. Dabei geht es zum einen darum, Ärzte und Medizinstudenten so fortoder auszubilden, dass sie Patienten proaktiv in die Entscheidungsfindung für therapeutisches Vorgehen mit einbeziehen, und zum anderen Patienten durch Informationsmöglichkeiten in den Stand zu versetzen, Vor- und Nachteile abwägen zu können. Solche Portale zur Patientenbildung finden sich u. a. bei der ÄZQ (s. 5.5.2) und dem IQWIG (s. 5.4.2). Zum Beispiel bietet das IQWIG auf seiner Website „Gesundheitsinformation.de“ eine Entscheidungshilfe zur Mandelentzündung und Tonsillektomie bei Kindern an [156].

Der Umsetzungsgrad der partizipativen Entscheidungsfindung zeigt allerdings noch Verbesserungspotenzial [157].

\subsubsection{Prozessoptimierungen}

Sie haben oft das Ziel, die Sicherheit für Patienten zu erhöhen oder Unannehmlichkeiten wie Wartezeiten zu reduzieren.

\subsubsection{Feedback}

Das Messen der Patientenzufriedenheit durch vor Ort ausfüllbare Feedback-Bögen oder durch systematische, postalische Befragungen gehört genauso zum Repertoire der meisten Gesundheitsorganisationen wie ein Beschwerdemanagement, das als solches nach außen deutlich erkennbar ist und eine zeitgerechte Beantwortung garantiert.

Regelmäßige Auswertungen des Patientenfeedbacks erzeugen Ergebnisparameter für das Qualitätsmanagement.

\subsection{Mitarbeiterorientierung}

Mitarbeiterorientierte Führung bedeutet, Bedürfnisse und Wünsche der Mitarbeiter ernst zu nehmen und im Führungsverhalten zu berücksichtigen. Dazu gehört Anerkennung und Wertschätzung für geleistete Beiträge, ein explanatorischer, dialogischer Führungsstil mit Teambesprechungen, Townhallmeetings usw., die Möglichkeit zum Erweitern der fachlichen Kompetenz durch interne und externe Fortbildungen, und das Fördern des eigenverantwortlichen Arbeitens durch Übertragen von Verantwortung („Empowerment“). Ziel der Mitarbeiterorientierung ist, über die so generierte Zufriedenheit langfristig die Qualität der Arbeitsergebnisse zu erhalten und zu steigern sowie qualifizierte Mitarbeiter zu binden.

Regelmäßiges Erfassen der Mitarbeiterzufriedenheit und der Fluktuationsrate erzeugt Indikatoren für die Ergebnisqualität.

\subsection{Kommunikation, Kooperation und Interdiszipli- narität}

Gute Kommunikation und Kooperation sorgen dafür, dass an Schnittstellen behandlungsrelevante Informationen vollständig übermittelt werden und das in interdisziplinären ständigen Konferenzen wie z. B. den Tumor-Boards oder in krankheitsorientierten Zentren (s. 4.7.2) durch Bündeln der Kompetenzen bestmögliche Entscheidungen getroffen werden können. Best-Practice-Initiativen geben weiterhin Anregungen zur Qualitätssteigerung.

\subsection{Einrichtungsübergreifende Initiativen}

Es gibt eine Vielzahl an freiwilligen einrichtungsübergreifenden Initiativen, die mit unterschiedlicher Herangehensweise versuchen, die Qualität im Behandlungsprozess zu verbessern. 


\subsubsection{Kollegiale Beratung - Qualitätszirkel und ärztliches} Peer Review

Zur kollegialen Beratung kann man sowohl die praxisübergreifenden Qualitätszirkel als auch das sog. ärztliche Peer-Review zählen. Letzteres wird durch nach BÄK-Kriterien ausgebildete Peers durchgeführt, die nach einem Vor-Ort-Besuch im Rahmen eines kollegialen Dialogs ein wertungs- und sanktionsfreies Feedback geben. Das formale Vorgehen gleicht mit standardisierter Selbst- und Fremdbewertung teilweise einer Zertifizierung, wobei der Schwerpunkt auf der Beratung liegt [158].

\subsubsection{Einrichtungsübergreifende Fehlermeldesysteme}

Einrichtungsübergreifende Fehlermeldesysteme bzw. Critical Incident Reporting Systems (CIRS) unter dem Dach des „Netzwerk CIRSmedical.de“" wurden bereits dargestellt (s. 5.5.2 und 5.2.5.5) [129]. Für Hausarztpraxen wurde das CIRS „Jeder Fehler zählt“ eingerichtet [159]. CIRS tragen wesentlich zur Identifikation von systembedingten Fehlermöglichkeiten bei (d. h. sog. latenten Konditionen nach Reason, s. 6.6.2).

\subsubsection{APS - Aktionsbündnis Patientensicherheit}

Das Aktionsbündnis Patientensicherheit (APS) ist eine Initiative von Berufsverbänden, Patientenvertretern, Organisationen der Selbstverwaltung des Gesundheitswesens und der Industrie, um die Sicherheit von Patienten im Behandlungsprozess zu erhöhen, indem durch sog. Handlungsempfehlungen versucht wird, systembedingte Fehlermöglichkeiten (d. h. sog. latente Konditionen nach Reason, s. 6.6.2) zu minimieren [125].

\subsubsection{IQM - Initiative Qualitätsmanagement}

\subsubsection{Geschichte und Organisation}

Die Initiative Qualitätsmedizin (IQM) ist eine freiwillige Initiative von Krankenhäusern aus Deutschland und der Schweiz mit dem Ziel, die Qualität in den angeschlossenen Krankenhäusern zu verbessern. Sie wurde im Jahr 2008 von privaten und gemeinnützigen Klinikketten, mehreren Universitätsklinika und der Ärztekammer Berlin gegründet.

In Kooperation mit dem wissenschaftlichen Institut der AOK (WIdO) wurden Qualitätsindikatoren aus Routinedaten, d. h. den zur Abrechnung der DRGs übermittelten ICD und OPS, entwickelt (sog. Inpatient Quality Indikators, IQI). Durch die Zusammenarbeit mit der AOK werden auch sektorenübergreifende Verlaufsdaten ausgewertet. Der Vorteil laut IQM ist, dass diese Daten ohne zusätzlichen Aufwand zur Verfügung stehen, anders als bei externen Zertifizierungsverfahren.

\subsubsection{Verfahren}

Insgesamt 40 Qualitätsindikatoren wurden definiert. Die Ergebnisse werden in jährlichen Berichten von den Krankenhäusern publiziert und enthalten z. B. Angaben zur Mortalität bei bestimmten Diagnosen oder Prozeduren in Relation zum Durchschnitt der IQMKrankenhäuser und zum risikoadjustierten Erwartungswert. Die HNO-Heilkunde ist nicht mit einem Qualitätsindikator, wohl aber mit der durchschnittlichen stationären Aufenthaltsdauer nach Tonsillektomie repräsentiert. Die sektorenübergreifenden Ergebnisse werden im AOK-Krankenhausnavigator veröffentlicht.
Bei auffälligen Abweichungen wird ein Peer-Review, in Deutschland unter Inanspruchnahme des BÄK-Verfahrens (s. 6.10.1), zur Qualitätsverbesserung eingeleitet. Eine Zertifikatsvergabe o.ä. findet nicht statt [160].

\subsubsection{Relevanz}

Auf der Website der IQM sind derzeit 447 teilnehmende Krankenhäuser aus Deutschland aufgeführt, darunter mehrere Universitätsklinika. In einer Studie wurde eine statistisch signifikante Verbesserung der Qualität bei IQM-Kliniken, die sich dem Peer-Review nach auffälligen IQI unterzogen hatten, festgestellt [161]. Dadurch hat die IQM ein Alleinstellungsmerkmal im Vergleich zu anderen externen Verfahren (s. 4.8, 5.12 und 8.4)

\subsection{5 ÄQSI - Datenbank ärztlicher Qualitätssicherungsinitia- tiven}

Die Bundesärztekammer hat eine Online-Datenbank eingerichtet, die freiwillige ärztliche Qualitätssicherungsinitiativen erfasst, deren Ziel das Messen und Verbessern der Behandlungsqualität ist. Darin eingeschlossen sind auch Register zu bestimmten Krankheitsbildern und Implantaten. Die Datenbank soll einen Überblick über diese Initiativen geben und Interessierten somit die Möglichkeit, sich einer Initiative seines Fachgebiets anzuschließen [162].

Insgesamt werden 147 Initiativen aufgeführt, davon 2 vom Berufsverband HNO („Audiologie/Neurootologie“ und „Qualitätssiegel Allergologie“). Ein Register für Cochlea Implantate ist in Vorbereitung und sollte zukünftig auch in der ÄQSI aufgeführt sein.

\subsubsection{Leitlinien}

Leitlinien sind ein wesentlicher Bestandteil des Wissensmanagements in der Medizin. Sie sind nach einem dedizierten Regelwerk entwickelt und geben den gegenwärtigen Kenntnisstand zu einem Thema wieder, um Ärzte, aber auch Patienten bei der Entscheidungsfindung zu unterstützen. Sie sind dadurch ein wichtiges Instrument der Sicherung und Fortentwicklung der medizinischen Behandlungsqualität. Leitlinien zeichnen sich dadurch aus, dass sie handlungsorientiert sind, d. h. „Handlungs- und Entscheidungskorridore " vorgeben, von denen im Einzelfall jedoch begründet abgewichen werden darf oder ggf. sogar muss.

Die AWMF gibt die Leitlinien der medizinischen Fachgesellschaften heraus. Sie hat ein Regelwerk bezüglich der Leitlinienentwicklung erstellt und gewährleistet dessen Umsetzung durch administrative Betreuung und Supervision. Zur Beurteilung des Qualitätsstandards von neuen Leitlinien wird das „Deutsche Instrument zur methodischen Leitlinien-Bewertung“ (DELBI) angewandt. Leitlinien werden bei Veröffentlichung mit einem Ablaufdatum versehen [163].

Leitlinien werden in 4 Klassen unterschieden:

- S1: Handlungsempfehlung von Experten (Konsensfindung in einem informellen Verfahren)

- S2k (vormalig S2): Konsensbasierte Leitlinie (Repräsentatives Gremium, strukturierte Konsensfindung)

- S2e: Evidenzbasierte Leitlinie (Systematische Recherche, Auswahl, Bewertung von Literatur)

- S3: Evidenz- und konsensbasierte Leitlinie (kombinierte Voraussetzungen von S2k und S2e) 
Die DGHNO-KHC hat derzeit 19 aktuelle eigene Leitlinien veröffentlicht und ist an 57 Leitlinien anderer Fachgesellschaften beteiligt.

Während es einerseits Hinweise auf den positiven Einfluss von Leitlinien auf die medizinische Ergebnisqualität gibt [164, 165], wird andererseits kritisch angeführt, dass bedingt durch das oft mehrjährige Erstellungsverfahren die Aktualität nicht in jedem Fall gegeben sein mag, oder dass sich Leitlinien im internationalen Vergleich inhaltlich widersprechen. Das Qualitätsniveau der deutschen Leitlinien wurde durch ein Clearingverfahren Anfang des Jahrtausends deutlich angehoben [166].

Neben den von der AWMF herausgegebenen Leitlinien der Fachgesellschaften gibt es noch die Nationalen Versorgungsleitlinien (s. 5.5.2) und die gemeinsam mit der Deutschen Krebsgesellschaft und der Deutschen Krebshilfe herausgegebenen Onkologischen Leitlinien [167].

\subsubsection{KE - Klug Entscheiden (Choosing Wisely Initiative)}

Die „Choosing Wisely Initiative“ (CWI) hat ihren Ursprung in den USA. Als Reaktion auf Berechnungen, dass mindestens $20 \%$ der medizinischen Leistungen mit einem Volumen von jährlich bis zu 200 Mrd. US-\$ überflüssig („waste“ und „no value“) seien [168], formulierte der Allgemeinarzt Howard Brody die CWI: jede Fachdisziplin solle eine Top-5-Liste von überflüssigen Prozeduren zusammenstellen und diese mit einer negativen Empfehlung versehen. Als Kriterien zur Aufnahme in die Liste nannte er Häufigkeit, Kostspieligkeit und fehlende Evidenz. Als zusätzliche Begründung wurde später angeführt, dass die CWI auch vermeidbaren Schaden vom Patienten abwenden könne (z. B. Strahlenbelastung). Mit Unterstützung des American Board of Internal Medicine (ABIM) wurde daraus eine nationale und internationale Bewegung mit mehr als 20 beteiligten Nationen.

Es gibt erhebliche Kritik an der internationalen CWI: Im Sinne eines „mission creep“ sollen jetzt statt „no value“ auch „low value“ Prozeduren auf die Negativlisten aufgenommen werden, was der zuvor heftig bestrittenen Rationierungsdiskussion, im Wording auch als Priorisierung oder Posteriorisierung verbrämt, neuen Auftrieb gibt, jedoch nicht transparent kommuniziert wird. Ganz im Gegenteil wird das Patientenwohl als alleinige Motivation angeführt. Versorgungspolitische Aspekte, die man durchaus legitim diskutieren könnte, werden jedoch ausgeblendet. Weiterhin erreichen die Empfehlungen bei Weitem nicht die Standards, die für die Entwicklung von Leitlinien gelten. Und schließlich gibt es derzeit keine Hinweise auf die Wirksamkeit der Initiative, einschließlich der Beantwortung der Frage nach der Motivation der angesprochenen Ärzte, den Empfehlungen auch zu folgen $[169,170]$. In einer aktuellen Studie konnte für die USA gezeigt werden, dass bevorzugt Prozeduren von anderen Fachdisziplinen auf die Negativlisten gesetzt wurden [171].

In Deutschland wird die CWI als „Klug Entscheiden“ (KE) von der Deutschen Gesellschaft für Innere Medizin (DGIM) unter Mitbeteiligung von 12 Fachgesellschaften betrieben [172]. Als einzige der international beteiligten Organisationen formuliert sie auch PositivEmpfehlungen bei identifizierter Unterversorgung. Die AWMF hat ein Manual mit qualitativen Mindestanforderungen für die Entwicklung von KE-Empfehlungen erarbeitet [173]. Die Deutsche Gesellschaft für Hals-Nasen-Ohren-Heilkunde, Kopf- und Hals-Chirurgie (DGHNO-KHC) hat sich bislang nicht an der KE-Initiative beteiligt.
Mögliche Gründe für ärztliche Entscheidungen, die zu Über- und Unterversorgung führen, werden weiter unten diskutiert (s. 8.1.5)

\subsubsection{EBM - Deutsches Netzwerk evidenzbasierte Medizin}

\subsubsection{Evidence Based Medicine}

Evidence Based Medicine (EBM) ist eine Mitte der neunziger Jahre des letzten Jahrhunderts entstandene Bewegung. David Sackett hat 1996 in einem Editorial eine prägnante Beschreibung gegeben, was EBM ist - und was sie nicht ist:

„EBM ist der gewissenhafte, ausdrückliche und vernünftige Gebrauch der gegenwärtig besten externen, wissenschaftlichen Evidenz für Entscheidungen in der medizinischen Versorgung individueller Patienten. Die Praxis der EBM bedeutet die Integration individueller klinischer Expertise mit der bestmöglichen externen Evidenz aus systematischer Forschung “.

Er betont also, dass die klinische Expertise gleichrangig mit der externen Evidenz anzusehen ist und nur im Zusammenspiel der beiden die beste Versorgungsqualität erreicht werden kann. EBM sei also keine ausschließlich nach externen Evidenzen „kochbuchartig“ auszuführende Medizin. Er stellt weiterhin klar, dass EBM nicht auf den „Goldstandard“ der Auswertung von randomisierten, kontrollierten Studien (RCT) begrenzt ist und sein darf. Auch Querschnittstudien, Follow-up-Studien, Erkenntnisse aus Grundlagenwissenschaften oder die „nächstbeste externe Evidenz“ können sinnvoll und angemessen sein - und für Interventionen, die einen ansonsten fatalen Ausgang verhindern würden, erübrigten sich kontrollierte Studien selbstverständlich [174] (deutsche Übersetzung aus [175]). Die Anwendung von EBM auf chirurgische Interventionen wird weiter unten diskutiert (s. 8.3.2).

\subsubsection{Cochrane}

Einen wichtigen Beitrag zur EBM leistet die Cochrane Collaboration, die ein weltweites Netz von Wissenschaftlern darstellt, die „systematische Reviews von primärer Forschung zu menschlicher Gesundheitsversorgung“" erstellen [176]. Auf der öffentlich zugänglichen Datenbank können Reviews nach Fragestellungen und Themengebieten gesucht werden. Für die HNO-Heilkunde sind derzeit 187 Reviews gelistet [177].

\subsubsection{Deutsches Netzwerk Evidenzbasierte Medizin}

Das Deutsche Netzwerk Evidenzbasierte Medizin (DNEbM) ist das deutschsprachige Kompetenz- und Referenzzentrum für alle Aspekte der Evidenzbasierten Medizin. Erklärtes Ziel ist, Konzepte und Methoden der EBM in Praxis, Lehre und Forschung zu verbreiten und weiter zu entwickeln [178].

\subsubsection{DNVF - Deutsches Netzwerk Versorgungsforschung}

Das „Deutsche Netzwerk Versorgungsforschung“ (DNVF) ist „eine Plattform von Fachgesellschaften, Institutionen und Personen mit dem besonderen Anliegen der Erforschung der Gesundheits- und Krankenversorgung“ um „wichtige Voraussetzungen für evidenzbasierte Entscheidungen im Gesundheitswesen sowie eine verbesserte Versorgung und Gesundheit der Bevölkerung “ zu schaffen. Ein besonderer Fokus liege dabei auf der Patientenperspektive [179]. 


\subsection{Diskussion - eine extensive Qualitätslandschaft}

Das Gewährleisten einer hohen Qualität der medizinischen Versorgung gehört zum professionellen Ethos der deutschen Ärzteschaft. Schon lange vor Beginn gesetzlicher Verpflichtungen und derzeit weit über diese hinaus drückt sich das nicht nur in der täglichen Praxis, sondern in zahlreichen freiwilligen Initiativen aus, die teils unabhängig organisiert sind und teils von den Institutionen der Ärzteschaft getragen werden. Dadurch ist jenseits der obligatorischen gesetzlichen Qualitätssicherung eine extensive Qualitätslandschaft entstanden.

\section{Stand des Qualitätsmanagements in deut- schen Krankenhäusern und Praxen, insb. in der HNO-Heilkunde}

Im Bereich der externen Qualitätssicherung und der Qualitätsberichte werden bei Krankenhäusern die gesetzlichen und vom G-BA spezifizierten Vorgaben unter Androhung von Sanktionen durchgesetzt. Anders liegt der Fall bei der Qualitätsmanagementrichtlinie des G-BA (QM-RL), die Krankenhäuser, Praxen und MVZs seit fast 15 Jahren verpflichtet, ein umfassendes internes Qualitätsmanagementsystem zu betreiben. Dies wird bei Krankenhäusern nicht kontrolliert. Lediglich zu einzelnen Aspekten des Risikomanagements müssen im Qualitätsbericht Angaben gemacht werden. Im vertragsärztlichen Bereich erfolgt eine stichprobengestützte, jährliche Umfrage zu diesem Thema.

In Zeiten großer Herausforderungen (s. Kapitel 8) ergab sich die Frage, inwieweit diese an sich sinnvolle Vorgabe tatsächlich umgesetzt wird, zumal sie personelle und finanzielle Ressourcen bindet.

Zu diesem Zweck wurden im Juni 2019 Fragebögen an alle deutschen HNO-Kliniken und an die niedergelassenen HNO-Ärzte im Rhein-Neckar-Kreis versandt. Für die Auswertung wurden Rücksendungen bis Ende August 2019 berücksichtigt. Die Fragebögen wurden unter der Zielvorgabe, einen hohen Rücklauf zu erhalten, bewusst kompakt gehalten.

\subsection{Krankenhäuser - Externe Qualitätssicherung}

Dem aktuellen Qualitätsbericht des IQTIG über das Jahr 2017 ist zu entnehmen, dass die Krankenhäuser ihrer Verpflichtung zur Übermittlung der Daten für die Verfahren nach QSKH-RL zu über $99 \%$ nachkommen. Von den dabei zur Anwendung gekommenen Qualitätsindikatoren (QI) zeigten im Vergleich zum Vorjahr 17\% eine Verbesserung, $5 \%$ eine Verschlechterung und $67 \%$ keine Veränderung, der Rest war nicht vergleichbar. Bei $4 \%$ der QI bestand ein „ausgeprägtes oder fortbestehendes Qualitätsdefizit“ im bundesdeutschen Durchschnitt. Lediglich bei einem Prozent der aus den QI berechneten Ergebnissen lag tatsächlich eine relevante Auffälligkeit, bezogen auf einzelne Krankenhäuser, vor (weitere Einzelheiten siehe 5.2.6.4) [96]. Zusammenfassend sprechen diese Ergebnisse für ein sehr hohes Qualitätsniveau der Krankenhäuser - in Bezug auf die geprüften Kriterien, die jedoch nur einen Teilbereich abdecken. Wie bereits zuvor gesagt, ist die HNO-Heilkunde selbst von der externen Qualitätssicherung nach QSKH-RL nicht betroffen, weil keine Qualitätsindikatoren für unser Fachgebiet existieren.

Zu freiwilligen externen qualitätssichernden Verfahren sind folgende Angaben verfügbar:
Am IQM-Verfahren nehmen derzeit 447 Krankenhäuser teil, nach KTQ sind 244 Krankenhäuser zertifiziert, 14 nach EFQM und 2 nach JCI. ISO veröffentlicht keine Zahlen; es gibt Schätzungen über ca. 400 zertifizierte Krankenhäuser oder Organisationseinheiten von Krankenhäusern.

\subsection{HNO-Krankenhäuser - Ergebnisse der Umfrage}

Es wurden 168 Kliniken bzw. Hauptabteilungen angeschrieben, der Rücklauf betrug 128 Fragebögen (76\%). Die Kliniken wurden in 3 Gruppen eingeteilt: Kliniken mit weniger als 30 Betten (Rücklauf 34), Kliniken mit mehr als 30 Betten (Rücklauf 61) und Uni-Kliniken (Rücklauf 33).

Die 11 inhaltlichen Fragen waren in 5 Gruppen eingeteilt: Externe Zertifizierung, Standardisierung, Organisationsentwicklung, Risikomanagement und Patienten- und Mitarbeiterorientierung ( $\triangleright$ Tab. 1):

Bei den Fragen nach Zertifizierung ergaben sich 2 gegenläufige Trends: der Anteil der Gesamtzertifizierungen nimmt mit steigender Größe ab, der Anteil der Zentrumszertifizierungen nimmt zu. Ersteres erklärt sich aus dem (über-)proportional steigenden Aufwand mit der Zunahme der Größe und der Komplexität einer Klinik, letzteres ergibt sich aus der steigenden Kompetenz in der Maximalversorgung.

Die Fragen zur Standardisierung von bestimmten Behandlungsprozessen werden durchgehend zu einem hohen Anteil positiv beantwortet, und das Ergebnis der Frage nach Qualitätshandbüchern deckt sich mit dem der Zertifizierung, d. h. knapp $80 \%$ der Teilnehmer der Umfrage wenden offensichtlich Elemente eines internen Qualitätsmanagementsystems an.

Dies bestätigt sich auch bei der Frage nach Prozessoptimierungen (Qualitätszirkel), die von einem vergleichbaren Anteil positiv beantwortet wird.

Hauptbestandteil eines Qualitätsmanagementsystems ist das Risikomanagement. Erwartungsgemäß ergeben sich bei den von den Kliniken zu verantworteten Bereichen des Risikomanagements hohe Umsetzungsgrade von über $95 \%$.

Im gleichen Bereich finden sich die Ergebnisse für die Patientenorientierung, gegen die die Mitarbeiterorientierung leicht abfällt.

Zusammenfassend kann man feststellen, dass eine sehr hohe und annähernd homogene (mit Ausnahme der Zertifizierungen) Umsetzung von Qualitätsmanagement in HNO-Kliniken berichtet wird („Erreichungsgrad“). Über das Ausmaß des sog. „Durchdringungsgrades“ (Maß der Umsetzung in der Breite) lässt sich naturgemäß mit dem Mittel dieser Umfrage keine Aussage treffen.

\subsection{Praxen - Bericht der KBV zum Umsetzungsstand von Qualitätsmanagement}

Im aktuellen Bericht der KBV zum Umsetzungsstand von Qualitätsmanagement in Vertragsarztpraxen und MVZ, der auf einer Stichprobe von 2017 beruht, wird über einen insgesamt hohen Umsetzungsgrad - knapp $90 \%$ - von Methoden und Instrumenten des Qualitätsmanagements berichtet. Nach unten wichen davon ab Patientenbefragungen ( $54 \%$ ) sowie in geringerem Ausmaß Mitarbeiterbefragungen, Checklisten und Selbstbewertungen anhand von Qualitätszielen.

Zu freiwilligen externen qualitätssichernden Verfahren macht die KBV folgende Angaben: 
- Tab. 1 Ergebnisse der Umfrage bei Kliniken. Anmerkung zu Zeile 1: Da die Anzahl der Nicht-Unikliniken nur insgesamt bekannt war, aber nicht ihre Verteilung auf die beiden Gruppen mit weniger oder mehr als 30 Betten, erfolgte die prozentuale Bewertung des Rücklaufs als Gesamtgruppe mit $72 \%$.

\begin{tabular}{|c|c|c|c|c|c|c|c|c|}
\hline & $\begin{array}{l}\text { Alle } \\
\text { Kliniken }\end{array}$ & $\begin{array}{l}\text { prozen- } \\
\text { tual }\end{array}$ & $\begin{array}{l}<30 \\
\text { Betten }\end{array}$ & $\begin{array}{l}\text { prozen- } \\
\text { tual }\end{array}$ & $\begin{array}{l}>30 \\
\text { Betten }\end{array}$ & $\begin{array}{l}\text { prozen- } \\
\text { tual }\end{array}$ & $\begin{array}{l}\text { Uni- } \\
\text { Klinik }\end{array}$ & $\begin{array}{l}\text { prozen- } \\
\text { tual }\end{array}$ \\
\hline Rücklauf & 128 & $75,7 \%$ & 34 & $72 \% 1$ & 61 & $72,0 \% 1$ & 33 & $89,2 \%$ \\
\hline \multicolumn{9}{|l|}{ Zertifizierung } \\
\hline 1. Ist Ihre gesamte Klinik zertifiziert (z. B. KTQ, ISO, JCI..)? & 99 & $77,3 \%$ & 29 & $85,3 \%$ & 50 & $82,0 \%$ & 20 & $60,6 \%$ \\
\hline $\begin{array}{l}\text { 2. Ist Ihre Klinik als Teil eines Zentrums zertifiziert (z. B. } \\
\text { Schädelbasiszentrum)? }\end{array}$ & 85 & $66,4 \%$ & 13 & $38,2 \%$ & 42 & $68,9 \%$ & 30 & $90,9 \%$ \\
\hline \multicolumn{9}{|l|}{ Standardisierung } \\
\hline 3. Verwenden Sie SOPs`? & 119 & $93,0 \%$ & 28 & $82,4 \%$ & 60 & $98,4 \%$ & 31 & $93,9 \%$ \\
\hline 4. Verwenden Sie Behandlungspfade? & 117 & $91,4 \%$ & 30 & $88,2 \%$ & 58 & $95,1 \%$ & 29 & $87,9 \%$ \\
\hline 5. Haben Sie ein oder mehrere Qualitätshandbücher erstellt? & 102 & $79,7 \%$ & 27 & $79,4 \%$ & 47 & $77,0 \%$ & 28 & $84,8 \%$ \\
\hline \multicolumn{9}{|l|}{ Organisationsentwicklung } \\
\hline 6. Setzen Sie für bestimme Projekte Qualitätszirkel ein? & 105 & $82,0 \%$ & 26 & $76,5 \%$ & 49 & $80,3 \%$ & 30 & $90,9 \%$ \\
\hline \multicolumn{9}{|l|}{ Risikomanagement } \\
\hline $\begin{array}{l}\text { 7. Gibt es in Ihrer Klinik Fehlerkonferenzen (oder M\&M } \\
\text { Konferenzen)? }\end{array}$ & 122 & $95,3 \%$ & 33 & $97,1 \%$ & 58 & $95,1 \%$ & 31 & $93,9 \%$ \\
\hline $\begin{array}{l}\text { 8. Gibt es in Ihrer Klinik/Klinikum ein CIRS (Critical Incident } \\
\text { Reporting System?) }\end{array}$ & 124 & $96,9 \%$ & 33 & $97,1 \%$ & 59 & $96,7 \%$ & 32 & $97,0 \%$ \\
\hline \multicolumn{9}{|l|}{ Patienten- und Mitarbeiterorientierung } \\
\hline 9. Setzen Sie ein Beschwerdemanagement ein? & 128 & $100,0 \%$ & 34 & $100,0 \%$ & 61 & $100,0 \%$ & 33 & $100,0 \%$ \\
\hline 10. Führen Sie Patientenbefragungen durch? & 124 & $96,9 \%$ & 32 & $94,1 \%$ & 60 & $98,4 \%$ & 32 & $97,0 \%$ \\
\hline 11. Führen Sie Mitarbeiterbefragungen durch? & 107 & $83,6 \%$ & 28 & $82,4 \%$ & 53 & $86,9 \%$ & 26 & $78,8 \%$ \\
\hline
\end{tabular}

Während Instrumente der externen Zertifizierung innerhalb des Qualitätsmanagementsystems der Praxen zu $54 \%$ angewendet wurden, haben sich nur $13 \%$ einer zusätzlichen externen Zertifizierung unterzogen (s. 5.2.6.2), d. h. dreiviertel der Anwender belassen es bei der Selbstbewertung.

\subsection{HNO-Praxen - Ergebnisse der Umfrage}

Es wurden 56 Praxen in der Rhein-Necker-Region angeschrieben, der Rücklauf betrug 29 Fragebögen (53\%).

Die 14 inhaltlichen Fragen waren in 5 Gruppen eingeteilt: Externe Zertifizierung, Standardisierung, Organisationsentwicklung, Risikomanagement und Patienten- und Mitarbeiterorientierung ( $\triangleright$ Tab. 2).

Ein Viertel der antwortenden Praxen gab an, zertifiziert zu sein.

Die Standardisierung von Prozessen durch Checklisten und Qualitätshandtücher weist einen sehr hohen Umsetzungsgrad auf. Bei der Frage nach OP-Checklisten ist zu berücksichtigen, dass viele Praxen keine operativen Eingriffe durchführen.

Die Prozessoptimierungen betreffende Frage nach Qualitätszirkeln wurde mit knapp $40 \%$ positiv beantwortet. Dabei ist zu bedenken, dass im überschaubaren Personenkreis einer Praxis Prozessoptimierungen auch in Mitarbeiterbesprechungen angestoßen werden können.

Fragen, die die Patientensicherheit betreffen, zeigten durchgängig einen hohen Umsetzungsgrad. Das betrifft die Festlegung von Verantwortlichkeiten, Teambesprechungen, Geräteeinweisungen und ein dediziertes Risiko- und Fehlermanagement. Ungefähr die Hälfte der Praxen wurde schon einmal einer externen Hygienebegehung unterzogen.

Instrumente der Patienten- und Mitarbeiterorientierung zeigten einen geringeren Umsetzungsgrad als in den Kliniken, wurden aber immer noch in der Mehrheit der Praxen angewandt. In einer Praxis besteht allerdings auch eher die Möglichkeit zu einem unmittelbaren, nicht durch eine Umfrage erhobenem Feedback von Patienten und Mitarbeitern als in einer Klinik.

Zusammenfassend kann man festhalten, dass trotz der im Vergleich zu den Kliniken deutlich niedrigeren Zertifizierungsrate innerhalb der Praxen Instrumente des Qualitätsmanagements zu einem hohen Grad Anwendung finden.

\subsection{Diskussion - gute Ergebnisse für die HNO- Heilkunde}

\subsubsection{Vergleich von HNO-Kliniken mit der deutschen} Krankenhauslandschaft

Um den Stand des Qualitätsmanagements von HNO-Kliniken mit der gesamten deutschen Krankenhauslandschaft zu vergleichen, gibt es nur einen Parameter: Den Umfang der externen Zertifizierung.

Das statistische Bundesamt gibt die Zahl aller deutschen Krankenhäuser für 2017 mit 1942 an. Davon hatten 1834 ein Leistungsspektrum, das sie verpflichtet hat, an der externen Qualitätssicherung (QSKH-RL) teilzunehmen [96]. Setzt man die Summe der zertifizierten Kliniken nach KTQ (244), EFQM (14), JCI (2) und ISO (ca. 
- Tab. 2 Ergebnisse der Umfrage bei Praxen. Anmerkung zu Frage 3: Da nicht separat abgefragt wurde, ob die Praxis operative Eingriffe durchführt, ist das Ergebnis dieser Frage nicht repräsentativ.

\begin{tabular}{|c|c|c|}
\hline & Praxen & prozentual \\
\hline Rücklauf & 29 & $52,7 \%$ \\
\hline \multicolumn{3}{|l|}{ Zertifizierung } \\
\hline 1. Ist ihre Praxis zertifiziert? & 7 & $24,1 \%$ \\
\hline \multicolumn{3}{|l|}{ Standardisierung } \\
\hline 2. Verwenden Sie Checklisten? & 28 & $96,6 \%$ \\
\hline 3. Verwenden Sie OP-Sicherheits-Checklisten (time-out)? (s. Anm.) & 12 & $41,4 \%$ \\
\hline 4. Haben Sie ein Qualitätshandbuch? & 28 & $96,6 \%$ \\
\hline \multicolumn{3}{|l|}{ Organisationsentwicklung } \\
\hline 5. Sind Verantwortlichkeiten eindeutig bzw. schriftlich festgelegt? & 26 & $89,7 \%$ \\
\hline 6. Führen Sie regelmäßig Teambesprechungen durch? & 27 & $93,1 \%$ \\
\hline 7. Setzen Sie für bestimmte Projekte Qualitätszirkel ein? & 11 & $37,9 \%$ \\
\hline \multicolumn{3}{|l|}{ Risikomanagement } \\
\hline 8. Hatten Sie schon mal eine externe Hygiene-Begehung (RP, Gesundheitsamt)? & 16 & $55,2 \%$ \\
\hline 9. Führen Sie dokumentierte Geräteeinweisungen durch? & 28 & $96,6 \%$ \\
\hline 10. Gibt es in Ihrer Praxis ein Risikomanagement? & 24 & $82,8 \%$ \\
\hline 11. Gibt es in Ihrer Praxis ein Fehlermanagement? & 24 & $82,8 \%$ \\
\hline \multicolumn{3}{|l|}{ Patienten- und Mitarbeiterorientierung } \\
\hline 12. Setzen Sie ein Beschwerdemanagement ein? & 15 & $51,7 \%$ \\
\hline 13. Führen Sie Patientenbefragungen durch? & 19 & $65,5 \%$ \\
\hline 14. Führen Sie Mitarbeiterbefragungen durch? & 16 & $55,2 \%$ \\
\hline
\end{tabular}

400) in Relation zur Gesamtzahl aller Krankenhäuser, die der QSKHRL unterliegen, ergibt sich ein Zertifizierungsgrad von ca. $36 \%$. Das berücksichtigt nicht, dass Doppelzertifizierungen nach 2 Systemen nicht selten sind (z. B. ISO und KTQ) und dass ISO oft nur auf Organisationseinheiten, aber nicht ganze Krankenhäuser ausgerollt wird. Realistisch wohlwollend kann man also vermutlich von einem Drittel aller deutschen Krankenhäuser ausgehen, die extern zertifiziert sind. IQM deckt mit 447 Teilnehmern mittlerweile schon ein Viertel aller Krankenhäuser ab, stellt aber keine Zertifikate aus und ist daher für diese Betrachtung nicht relevant.

In Relation dazu ist der Anteil zertifizierter HNO-Kliniken erheblich höher: Bei Uni-Kliniken mit $60 \%$ fast doppelt so hoch, bei NichtUnikliniken mit über $80 \%$ zweieinhalbmal so hoch. Das hat sicher auch damit zu tun, dass HNO-Hauptabteilungen nur an Krankenhäusern ab einer gewissen Versorgungsstufe vertreten sind: statistisch sind sie nur in knapp 9\% aller deutschen Krankenhäuser vorhanden.

\subsubsection{Trend: ISO gewinnt, KTQ verliert drastisch}

KTQ hat in den Jahren bis 2009 einen kometenhaften Anstieg von teilnehmenden Krankenhäusern verzeichnet: zur Spitzenzeit waren fast 700 nach KTQ zertifiziert. Seitdem hat sie einen konstanten, drastischen Rückgang auf derzeit knapp 250 hinnehmen müssen. ISO, die keine Zahlen veröffentlicht, ist in einer Gegenbewegung laut Zertifizierern erfolgreicher. Als Grund werden die bessere Skalierbarkeit des ISO-Verfahrens, der hohe Dokumentationsaufwand bei KTQ und die höheren Kosten bei einer Re-Zertifizierung durch KTQ angeführt.

\subsubsection{Trend: Qualität minus Qualitätsmanagementsystem und Zertifikat}

Bezüglich der Anwendung von Verfahren mit freiwilliger Selbst- und Fremdbewertung zeichnet sich eine interessante Dynamik ab: Das IQM-Verfahren verzeichnet offenbar erheblichen Zulauf, während KTQ wie bereits dargestellt Einbrüche hinnehmen muss. Die Auswirkung auf ISO ist nicht beurteilbar. Der Vorteil des IQM-Verfahrens ist die Reduktion auf das Wesentliche: Als Qualitätsindikatoren werden Daten verwendet, die schon im Abrechnungsprozess erstellt werden mussten. Dafür nimmt man ggf. Einschränkungen bei der Validität der Indikatoren in Kauf. Aufwendige Schulungen von Mitarbeitern für ein externes Zertifizierungssystem, aufwendiges Erstellen von Selbstbewertungen, Qualitätshandbüchern usw. entfallen genauso wie die Kosten für eine externe Zertifizierung.

Ein einrichtungsinternes, dediziertes Qualitätsmanagementsystem muss ebenfalls nicht nachgewiesen werden. Nur bei Auffälligkeiten der Qualitätsindikatoren wird auf ein Audit-System zurückgegriffen und dafür das schlanke, aber qualifizierte Peer-Review-Verfahren der BÄK bzw. einiger LÄK verwendet. So ist eine kompetente, problemzentrierte und praxisorientierte Beratung sichergestellt, die für einzuleitende Maßnahmen eine hohe Relevanz hat. Auf ein offizielles Zertifikat wird bewusst verzichtet. Stattdessen wird die Mitgliedschaft in der IQM publiziert und die Auswer- 
tung der Qualitätsindikatoren durch jährliche Veröffentlichung transparent gemacht.

Die Kurskorrektur der KTQ (s. 4.3.5) zielt mit Betonung des Beratungsaspektes und Reduktion des Dokumentationsaufwandes in die gleiche Richtung wie das Konzept der IQM.

\subsubsection{Zwei Gruppen: Mit und ohne zertifiziertes Qualitäts- managementsystem}

Zusammenfassend kann man feststellen, dass auch 15 Jahre nach Beschluss der Richtlinie QM-RL zwei Drittel aller deutschen Krankenhäuser und ein Viertel aller HNO-Klinken über kein zertifiziertes Qualitätsmanagementsystem verfügen. Der Umsetzungsgrad von risiko- und patientenbezogenen Anteilen eines Qualitätsmanagementsystems wird auch in dieser Gruppe bestimmt hoch sein, während er in den anderen Bereichen wahrscheinlich eher heterogen ist.

\subsubsection{Vergleich der HNO-Praxen mit einer Stichprobe deutscher Praxen}

Aufgrund der von der KBV erhobenen Umfragedaten ist anders als bei den Krankenhäusern der Vergleich von HNO-Praxen zum bundesdeutschen Durchschnitt einfacher.

Ähnlich wie bei den HNO-Kliniken sind auch die HNO-Praxen zu einem höheren Anteil zertifiziert, nämlich zu 24\% im Vergleich zu $13 \%$ des Bundesdurchschnitts. Bei Checklisten war der Umsetzungsgrad in HNO-Praxen $10 \%$ höher, die übrigen Parameter des Risiko- und Fehlermanagements lagen in vergleichbaren Bereichen. Qualitätszirkel kamen in HNO-Praxen nur ca. halb so oft zum Einsatz wie im bundesweiten Vergleich. Bei Patienten- und Mitarbeiterorientierung ergab sich ein heterogenes Bild: HNO-Praxen führen $10 \%$ mehr Patientenbefragungen durch, aber $30 \%$ weniger Mitarbeiterbefragungen und sie verfügen zu 30 \% weniger über ein Beschwerdemanagement.

Zur Vergleichbarkeit beider Umfragen ist zu sagen, dass die Stichproben ungefähr gleich groß waren (ca. 2\%), der Rücklauf der KBV-Umfrage allerdings fast doppelt so hoch (97\%) wie bei der HNO-Umfrage. Der größte Unterschied liegt darin, dass HNO-Facharztpraxen gegen einen Querschnitt aus Hausarztpraxen und Facharztpraxen aller Disziplinen verglichen werden mussten. Die KBV differenziert in ihrer Berichterstattung nicht zwischen Hausarztund Facharztpraxen oder gar einzelnen Facharztdisziplinen.

\section{Aktuellen Herausforderungen an Qualitäts- sicherung und -management}

Wie bereits ausgeführt, entsteht das eigentliche Qualitätsniveau der Behandlung am Ort der Versorgung in Krankenhäusern und Praxen. Herausforderungen und Risiken für die Behandlungsqualität stehen deshalb in engem Zusammenhang mit allem, was die Leistungserbringer belastet, überfordert oder Fehlanreize setzt, oder den gerechten Zugang für Patienten zur Gesundheitsversorgung gefährdet.

\subsection{Ressourcenallokation und Patientenwohl}

Zum Kernbereich Ressourcenallokation und Patientenwohl beziehen sich die folgenden Aussagen auf die Stellungnahme der AWMF „Medizin und Ökonomie - Maßnahmen für eine wissenschaftlich begründete, patientenzentrierte und ressourcenbewusste Versorgung “ von 2018 [143], das Gutachten des Sachverständigenrates Gesundheit (SVR) „Bedarfsgerechte Steuerung des Gesundheitswesens “ von 2018 [180] und die Stellungnahme des Deutschen Ethikrates „Patientenwohl als ethischer Maßstab für das Krankenhaus" von 2016 [181].

\subsection{1 Ökonomisierung versus Patientenwohl}

Sowohl nach Ansicht der AWMF als auch des Deutschen Ethikrates kommt es unter den derzeitigen Rahmenbedingungen zu Konflikten zwischen betriebswirtschaftlichen Anforderungen und einer evidenzbasierten, patientenorientierten Versorgung, die berufsethischen Ansprüchen genügt. Es sei eine Patientenselektion und Mengenausweitung standardisierbarer Eingriffe festzustellen. Das werfe nicht nur Fragen nach der individuellen Rechtfertigung der Indikationsstellung auf, sondern es gehe zu Lasten von Patientengruppen mit hohem Versorgungsaufwand, der Kinderheilkunde und der Geburtshilfe. Versorgungsgerechtigkeit und Patientenwohl seien gefährdet $[143,181]$.

In einer 2017 durchgeführten Befragung berichteten Krankenhausärzte von wachsendem Druck und indirekten und direkten Einflussnahmen, betriebswirtschaftliche Interessen bei patientenbezogenen Entscheidungen zu berücksichtigen. Dies führe zu UnterÜber- und Fehlversorgungen von Patienten, ethischen Konflikten, Stresssituationen und Frustration [182].

Die Ursachen für diese Situation werden in den folgenden 2 Kapiteln erörtert.

\subsubsection{Diagnosis Related Groups (DRG) - von der sprechen- den zur dokumentierenden Medizin}

Die 2004 verbindlich eingeführten DRGs sind das System, durch das überwiegend die Betriebskostenfinanzierung der deutschen Krankenhäuser erfolgt. Es hat in seiner nationalen Adaptation mittlerweile erhebliche Unzulänglichkeiten offenbart.

\subsubsection{Auswirkungen des DRG-Systems}

Seit Einführen der DRGs ist es zu einem deutlichen Anstieg der Fallzahlen gekommen, der fast ausschließlich elektive Prozeduren betrifft (z. B. [183]). Gleichzeitig ist es gegenläufig zur Verkürzung der Verweildauern gekommen. Da die reinen Vorhaltekosten der Krankenhäuser nicht gedeckt seien, gebe es Fehlanreize zu Leistungsbzw. Portfolioänderungen, „Rosinenpickereien“ und zum Aufsplitten komplexer Fälle [143]. Der ursprünglich intendierte Einsparungseffekt der DRGs wurde durch die Mengenausdehnungen aufgehoben. Leidtragende dieser Entwicklung seien finanziell unattraktive Patientengruppen (multimorbide, pädiatrisch usw.). Sprechende Medizin und Interdisziplinarität würden nicht vergütet, ebenso wenig wie Extremkostenfälle und Innovationen in der Hochleistungsmedizin [181]. So wird z. B. bei der interdisziplinären endoskopischen 4-Hand-Chirurgie der vorderen Schädelbasis durch HNO- und Neurochirurgen die Präsenz von 2 hochqualifizierten Operateuren nicht abgebildet.

Laut SVR sind die Fehlentwicklungen für Universitätskliniken und Maximalversorger besonders gravierend: Die deutsche DRGImplementation verfolge den „Einhaus-Ansatz“, d. h. anders als in Nachbarländern werde nicht zwischen (Krankenhaus-) Versorgungsstufen unterschieden, obwohl die Angebote nach Komplexi- 
tät, Menge und Vorhaltungskosten deutlich unterschiedlich seien. Das derzeitige DRG-System versuche dies zu kompensieren durch eine starke Ausdifferenzierung des DRG-Systems, ergänzt um Zuund Abschläge, und eine starke Prozedurenorientierung, die den Charakter eines Einzelleistungssystems annehme und dadurch Anreize zur Fallzahlausweitung schaffe [180].

Trotzdem wird es nicht erreicht, die Kostenstrukturen von Maximalversorgern korrekt abzubilden. In der Folge kommt es zu einer systematischen Unterfinanzierung der Universitätsklinken und Maximalversorger, die selbst leistungsstarke Häuser in eine negative Bilanz drückt.

\subsubsection{Vorschläge für Korrekturen der Betriebskostenfinanzie- rung}

Innerhalb des DRG-Systems sollten laut SVR Zuschläge bzw. Multiplikatoren nach Versorgungsstufen fachabteilungsbezogen eingeführt werden, um die Kostenstrukturen der Universitätskliniken und Maximalversorger abzubilden. Dadurch könnte das DRG-System wieder stärker auf Diagnose-bezogene Vergütung umgestellt werden und Fehlanreize würden zurückgehen. Dies entspricht den Forderungen von AWMF und Deutschen Ethikrat nach Zuschlägen für Universitätskliniken und Maximalversorger, ist aber der systemisch weitergehende und begrüßenswertere Vorschlag. Für mengenanfällige Elektiveingriffe wird ein verbindliches Zweitmeinungsverfahren durch Vertrauensärzte gefordert. Patienteninformation („sprechende Medizin“) und Aufwand für Interdisziplinarität sollten im System abgebildet werden, das Segmentieren von Fällen multimorbider Patienten durch „Paket-DRGs“ verhindert werden, für besonders aufwendige Patientengruppen müsse es Zuschläge geben und Extremkostenfälle müssten vergütet werden. Die AWMF fordert, dass DRG-Erlöse weder für Investitionen noch für Profite entnommen werden dürften und private Krankenhäuser deshalb nur als Non-Profit-Gesellschaften betrieben werden sollten.

Der Anteil pauschaler Vergütungselemente, z. B. für Vorhaltungsbedarf, außerhalb des DRG-Systems soll laut SVR und Deutschem Ethikrat deutlich erhöht werden. Bei Universitätskliniken solle für die Forschung ein ausgewogenes Verhältnis von Grundund Drittmitteln hergestellt werden [143, 180, 181].

\subsubsection{MDK- Fragwürdige Abrechnungsprüfungen}

Mehr als zweieinhalb Millionen Abrechnungsprüfungen hat der MDK 2018 an Krankenhäusern durchgeführt, was in den HNO-Kliniken einer Prüfquote von ca. $20 \%$ entspricht. Dabei geht es in der überwiegenden Mehrzahl nicht um falsche Kodierungen, sondern darum, durch Verkürzung der anerkannten Liegezeit Vergütungsabschläge zu Gunsten der Kassen zu generieren, obwohl die erbrachten medizinischen Leistungen unstrittig sind. Der administrative Aufwand der Prüfverfahren und der Aufwand der ärztlichen „Defensiv-Dokumentation“ sind erheblich - und im Wesen überflüssig. Erfreulicherweise soll diese fragwürdige Praxis demnächst vom Gesetzgeber korrigiert werden (MDK-Reformgesetz , s. 5.10).

\subsubsection{Investitionsmittel: too little, too late}

Im Rahmen der sog. dualen Finanzierung werden die Betriebskosten der Krankenhäuser von den Krankenkassen getragen und die Investitionskosten von den Bundesländern. In einer gemeinsamen
Pressemitteilung vom 21.03.2019 stellen DKG, Spitzenverband GKV und Verband der Privaten Krankenversicherung fest, dass aufgrund einer Investitionsanalyse des Instituts für das Entgeltsystem im Krankenhaus (InEK) der Investitionsmittelbedarf der deutschen Krankenhäuser 2019 bei über 6 Mrd. Euro liege, die Länder mit ihren Zahlungen aber nur knapp die Hälfte davon abdecken würden, da es keine verbindliche gesetzliche Regelung über das Volumen der Investitionsmittel gebe [184].

Dieses seit Jahren in gleicher Form bestehende starke Defizit der Investitionsfinanzierung ist mitverantwortlich für mangelnde Prozesseffizienz inkl. fehlender Digitalisierungskonzepte und veralteter Infrastruktur in den Krankenhäusern. In der Folge kommt es zur systemwidrigen Querfinanzierung aus DRG-Erlösen, auch weil Weiterentwicklungen in der Medizin Investitionen unabdingbar machen.

Während die AWMF eine prinzipielle Beibehaltung der dualen Finanzierung, nur in angemessener Höhe und mit Bundesbeteiligung fordert, schlägt der SVR perspektivisch die monistische Finanzierung durch die Kassen mit Steuerrückerstattung vor $[143,180]$.

Der derzeitige Zustand ist untragbar. Trotz wissenschaftlicher Evidenz für den tatsächlichen Investitionsbedarf enthalten die Länder den Krankenhäusern stoisch seit Jahren die notwendigen Mittel vor - mit erheblichen Auswirkungen für die Strukturqualität. Sie werden damit ihrer Verantwortung bedauerlicherweise nicht gerecht.

\subsubsection{Notfallversorgung - Patienten auf Abwegen}

Die Notfallversorgung beruht auf 3 Säulen: Dem kassenärztlichen Bereitschaftsdienst, dem Rettungsdienst und den Notfallambulanzen an Krankenhäusern. Seit Jahren besteht der Trend, dass der kassenärztliche Bereitschaftsdienst reduziert wird, indem z. B. der HNO-Bereitschaftsdienst eingestellt wird, und Patienten andererseits vermehrt direkt die Notfallambulanzen in Anspruch nehmen. Der SVR führt an, dass als Grund für das Aufsuchen der Notfallambulanzen bei nicht dringlichem Behandlungsbedarf die 24-stündige Verfügbarkeit, die ambulante, v. a. fachärztliche Versorgung oder auch die erhoffte gute Qualität der Behandlung bei interdisziplinärer Versorgung genannt würden [180].

Dadurch werden mit oft harmlosen Beschwerden spezialisierte Kapazitäten für wirklich bedürftige Patienten blockiert und teilweise grenzwertig lange Wartezeiten verursacht. Die gereizte, teils aggressive Stimmung der Wartenden ist eine Belastung für die Mitarbeiter. Für die Krankenhäuser sind die erhöhte Personalbindung und der Ressourceneinsatz defizitär, weil die Versorgung der Notfallpatienten nicht kostendeckend vergütet wird [185].

Der SVR schlägt vor, zur Lenkung der Notfallpatienten integrierte Leitstellen einzurichten und dazu die telefonischen Dienste unter den Nummern 116117 und 112 zu verschmelzen. Mit einer wie vom Marburger Bund und der KBV gemeinsam vorgeschlagenen „Strukturierten medizinische Ersteinschätzung in Deutschland“ (SmeD) könnte dann telefonisch den Patienten je nach Ergebnis das Aufsuchen einer Praxis, des kassenärztlichen Bereitschaftsdienstes oder einer Notfallambulanz empfohlen werden oder ggf. direkt der Rettungsdienst bestellt werden [186]. Das BMG hat durch die angekündigte zukünftige 24-stündige Erreichbarkeit der 116117 einen Schritt in diese Richtung getan. 
Der SVR greift weiterhin den Vorschlag der Deutschen Interdisziplinären Vereinigung für Intensiv- und Notfallmedizin (DIVI) auf [185], Integrierte Notfallzentren (INZ) an Krankenhäusern einzurichten, in denen die Notfallambulanzen und nach Möglichkeit der kassenärztliche Bereitschaftsdienst zusammengefasst sind (Portalpraxen). In ihnen könnten die Patienten je nach Schwere der Erkrankung ambulant behandelt oder der stationären Versorgung zugeführt werden. Die INZ sollen extrabudgetär vergütet werden. MB und KBV unterstützen diesen Vorschlag [187].

Diese Vorschläge erscheinen äußerst sinnvoll, ein belastendes Dauerthema der Krankenhäuser zu lösen, und sollten von einer breiten Patienteninformationskampagne zur Einführung begleitet werden.

\subsection{5 Überversorgung - Defensivmedizin und Patienten- wunsch}

Die Deutsche Gesellschaft für Innere Medizin (DGIM) hat 2017 im Rahmen ihrer Klug-Entscheiden-Initiative (s. 6.10.7) eine Mitgliederbefragung durchgeführt, welche Gründe es für Über- oder auch Unterversorgung gebe.

Die Mehrheit der Befragten war sich der negativen Folgen von Überversorgung bewusst, nämlich Steigerung der Gesundheitsausgaben, mögliche Verunsicherung und möglicher Schaden der Patienten. Die Frequenz von überflüssigen diagnostischen oder therapeutischen Maßnahmen wurde von einem Viertel der Befragten mit täglich und knapp der Hälfte mit mehrmals wöchentlich angegeben. Als Gründe wurden mit absteigender Häufigkeit Sorge vor Behandlungsfehlern (79\%), Druck der Patienten (63\%), Erzielung zusätzlicher Erlöse (48\%), Unkenntnis der Leitlinie (44\%), fehlende Zeit für den Patienten (24\%) u. a. angegeben. Als beste Gegenmaßnahme wurden Fortbildungsmaßnahmen genannt. Unterlassene, aber indizierte Leistungen wurden als deutlich kleineres Problem wahrgenommen [188].

Eine aktuelle Studie der American Medical Association (AMA) kommt bezüglich der Begründung für Überversorgung mit Angst vor Behandlungsfehler (85\%) und Druck von Patienten (59\%) zu ähnlichen Ergebnissen und gibt das Volumen der überflüssigen Prozeduren mit $20 \%$ an [189].

Die Schlussfolgerung der DGIM, als Gegenmaßnahmen gezielte Fortbildungsmaßnahmen und verbesserte inhaltliche Zugänglichkeit der Leitlinien, z. B. durch eine Executive Summary, abzustreben, erscheint sehr sinnvoll.

\subsubsection{Verteilungsgerechtigkeit und Partizipation}

Das Patientenwohl wird laut Deutschem Ethikrat durch Behandlungsqualität, Selbstbestimmung und Verteilungsgerechtigkeit bestimmt.

Die Selbstbestimmung solle durch partizipative Entscheidungsfindung (s. 6.7.2) sowie durch Stärkung der sprechenden Medizin im Krankenhaus über Vergütungsmodelle innerhalb des DRG-Systems gestärkt werden [181].

Die Verteilungsgerechtigkeit, die durch gleichen Zugang zu und die gerechte Verteilung von Ressourcen hergestellt wird, beruht zum einen auf dem Prinzip der zum sozialen Status indifferenten Versorgung nach Bedarf. Zum anderen besteht ein enger Zusammenhang zur Art der Verwendung der von der Gesellschaft aufgebrachten Ressourcen für das Gesundheitssystem. Über- oder Fehl- versorgung, ineffiziente und ineffektive Mittelverwendung sind ungerecht, weil verschwendete Mittel an anderer Stelle fehlen, an der sie sinnvoller hätten eingesetzt werden können [180].

Die Ressourcenallokation ist derzeit nach übereinstimmender Meinung nicht bedarfsgerecht. Fehlversorgungen sollten durch Gegensteuern gegen Fehlanreize im DRG-System korrigiert werden. Über- und Unterversorgung sollten durch bedarfsorientierte, sektorenübergreifende Planung mit regionaler Ausrichtung behoben werden, die sich an den zu erbringen Leistungen und weniger an Strukturen orientiert. Dabei sollte das Patientenwohl bundeseinheitlich durch einerseits wohnortnahe ambulante und stationäre Grundversorgung, andererseits durch spezialisierte Zentren gewährleistet werden. In die regionale Planung sollten alle beteiligten Mitglieder der Selbstverwaltung einbezogen werden.

Die Schnittstellenproblematik soll durch eine sektorenübergreifende elektronische Patientenakte adressiert werden. Der Ist-Zustand der Versorgung sollte regelmäßig überprüft werden und einer sektorenübergreifenden Qualitätssicherung unterliegen. Der Strukturfond zum Abbau von Krankenhaus-Überkapazitäten sollte fortgeführt werden, genauso wie die Unterstützung ländlicher Krankenhäuser durch den Sicherstellungszuschlag [143, 180, 181].

Dieses gestaffelte System der Versorgung steht in klarem Widerspruch zu dem Vorschlag der Bertelsmannstiftung, die Krankenhausversorgung auf ein Drittel der Standorte zu zentralisieren [190].

\subsection{Führung und Mitarbeiter im Krankenhaus}

\subsubsection{Führung - Balance von Medizin und Ökonomie}

Um die Fokussierung der Krankenhausleitungen auf das ökonomische Primat zu korrigieren, schlägt die AWMF vor, ärztliche Direktoren mit den kaufmännischen gleichzustellen und ein Wertemanagement inkl. eines Vetorechts von ärztlichen und pflegerischen Leitungen bei Entscheidungen, die die Versorgungsqualität oder Sicherheit beeinträchtigen könnten, einzuführen. Leistungsprämien in Chefarztverträgen sollten endgültig flächendeckend abgeschafft werden [143]. Der Deutsche Ethikrat empfiehlt, klinische Ethikkommissionen einzuführen.

\subsubsection{Mitarbeiter - In der Abwärtsspirale}

Durch die DRG-bedingten verkürzten Liegezeiten entstand bei annähernd unveränderten Personalschlüsseln ein erhöhter PatientenTurn-Over. Zusammen mit veränderten Arbeitszeiten aufgrund des Arbeitszeitgesetzes, vermehrten Teilzeitstellen und Pflegekräftemangel führte dies zu Zeitmangel und chronischer Überlastung, erhöhter Personalfluktuation und Krankenständen. Arbeitsverdichtung und zunehmende Bürokratisierung lassen immer weniger Zeit für den direkten Patientenkontakt. Das alles bedingte nicht nur abnehmende Branchenattraktivität und konsekutiven Fachkräftemangel, sondern birgt auch ein erhöhtes Gefährdungsrisiko für Patienten $[143,181]$. Die eingeleiteten Maßnahmen zur Pflegefinanzierung und zu Personalschlüsseln gehen in die richtige Richtung, weil sie mittelfristig durch Verbessern der Arbeitsbedingungen das Berufsbild der Pflege wieder attraktiver machen werden.

Unter den Bedingungen der Ökonomisierung und Arbeitsverdichtung wird auch die Kommunikationskompetenz zunehmend relevant. Die Sprachkompetenz sollte bei nicht-muttersprachlichen 
ärztlichen und pflegenden Mitarbeitern durch Deutschkurse und Fachsprachprüfungen durchgängig gewährleistet werden [181].

\subsubsection{Das Klima wird rauer}

Schlecht fassbar oder objektivierbar, hat sich in den letzten Jahren ein gesellschaftlicher Wandel vollzogen, der sich in einer veränderten Patientenhaltung ausdrückt. Ich-Bezogenheit und Anspruchshaltung nehmen zu, die Bereitschaft zu Geduld und Verständnis bei Unannehmlichkeiten nehmen ab. Gereiztheit und Aggression kulminieren besonders in Notfallambulanzen. Verbalinjurien, Sachbeschädigungen und sogar körperliche Gewalt gegen Mitarbeiter sind leider keine exotischen Ausnahmen mehr, sondern mittlerweile ein globales Phänomen. Das zeigt sich auch im Anstieg der diesbezüglichen Publikationen, die überwiegend erst nach 2010 verfasst wurden. Die Joint Commission veröffentlichte zu dem Thema einen „Sentinel Alert“ [191].

Es ist für Deutschland zu hoffen, dass durch die Reform der Notfallversorgung die Situation in den Notfallambulanzen entschärft werden kann. Kommunikationstraining für Deeskalationsstrategien und ggf. Präsenz von Sicherheitsdiensten sind an Brennpunkten sinnvoll. Der 122. Deutsche Ärztetag forderte den Gesetzgeber auf, den strafrechtlichen Schutz für Hilfeleistende zu erweitern [192].

\subsubsection{Arztwohl ist auch Patientenwohl}

Der Lancet proklamiert „Physician burnout: a global crisis“ [193]. In einer aktuellen Studie über deutsche Ärzte wird die Prävalenz von Burnout zwischen 4 und $20 \%$ und für Depression zwischen 6 und $13 \%$ geschätzt. Zusätzlich besteht Evidenz für Einschränkungen durch Angststörungen, suizidale Tendenzen und Substanzgebrauch. Berufsbezogene Stressfaktoren spielen eine wichtige Rolle bei der Entwicklung dieser Störungen [194].

Die im Genfer Gelöbnis enthaltene Verpflichtung, auf die eigene Gesundheit und das eigene Wohlergehen zu achten, sei graue Theorie, so der Bericht des Deutschen Ärzteblattes vom 122. Deutschen Ärztetag [195]. Unter den Bedingungen der Ökonomisierung mit Zeitdruck, Arbeitsverdichtung, Zunahme berufsfremder Tätigkeiten, insbesondere hoher Dokumentationsaufwand und Arbeit mit unergonomischen IT-Systemen, und Verlust an Handlungsautonomie sei Selbstfürsorge schwierig umzusetzen. Es gebe keine Kultur, Grenzen zu setzen und für Arztgesundheit einzustehen. Das Arbeitszeitgesetz wird immer noch nicht konsequent umgesetzt, obwohl übermüdete Ärzte eine erhebliche Patientengefährdung darstellen können.

In seiner Absurdität schon fast komisch mutet ein Versuch in den USA an, anstatt durch Verbesserung der Arbeitsbedingungen durch ein „Achtsamkeitstraining“ die Prävalenz von Burn-out unter Ärzten zu senken. Wie zu erwarten blieb durch die Intervention die Häufigkeit von Burn-out unverändert [196].

Der 122. Deutsche Ärztetagtag fordert Arbeitgeber, zuständige Behörden und Gesetzgeber zur Schaffung gesundheitsgerechter Arbeitsbedingungen für Ärztinnen und Ärzte auf. Insbesondere sei das Arbeitszeitgesetz einzuhalten [192].

Der ärztliche Personalmangel beruht auf der hohen Anzahl an Medizinstudium-Absolventen, die angesichts o.g. Bedingungen nicht klinisch tätig werden (relativer Ausbildungsmangel), weiterhin strukturell dadurch verschärft, dass bei steigendem Frauenanteil und der heute erfreulicherweise möglichen Verbindung von Familie und
Beruf die Lebensarbeitszeit der Ärztinnen durch mehrjährige Elternund Teilzeit deutlich reduziert ist. Mit dem Ausscheiden der BabyBoomer-Generation in die Rente wird sich die Situation weiter verschärfen. Der 122. Deutsche Ärztetag forderte deshalb, den Masterplan Medizinstudium 2020 vollständig umzusetzen und die Zahl der Medizinstudienplätze um 10\% zu erhöhen [192].

Die Weiterbildung von Assistenzärzten und die - insbesondere operative - Fortbildung von Fachärzten wird im derzeitigen System der Betriebskostenfinanzierung nicht refinanziert. Sie findet überwiegend an Universitätskliniken und Maximalversorgerhäusern statt. Unter den zuvor beschriebenen Bedingungen der Ökonomisierung leidet insbesondere die strukturierte Weiterbildung. Der SVR empfiehlt deshalb, einen Weiterbildungsfond einzurichten, aus dem personenbezogen die weiterbildenden Krankenhäuser vergütet werden (sog. Rucksackprinzip - der Weiterzubildende bringt seine Finanzierung mit) [180].

\subsubsection{Approbation bedeutungslos?}

In letzter Zeit gibt es Ansätze, die Approbation zu entwerten, indem nur Fachärzte als ausreichend kompetent deklariert werden. Beispiele sind die Vorschrift des Entlassmanagements, dass nur ein Facharzt das Rezept ausstellen darf, oder die marktradikale Krankenhausstudie der Bertelsmannstiftung, in der als Vorteil der Reduktion der Zahl der Krankenhäuser die Tatsache angeführt wird, dass dann auch nachts immer Fachärzte verfügbar seien.

Von Seiten unserer Berufsorganisationen sollte dieser Entwicklung entschieden entgegengetreten werden.

\subsection{Forschung, Wissenschaft und Fortschritt}

In Zeiten rapiden und zeitweise disruptiven Fortschritts auf den Feldern der „Omics“ (Gen-, Prote-, Microbi-...), der Ankündigung einer demnächst personalisierbaren bzw. individualisierbaren Therapie [197], eines aus DNA-Daten simulierten digitalen Zwillings und neuen aktiven Implantaten ist es eine Herausforderung, diese Entwicklungen auf ihren klinischen Nutzen zu bewerten. Dass das auch abgesehen von den Fällen plumpen Wissenschaftsbetrugs, in denen der Peer-Review-Prozess versagt hat, ein Prozess mit Irrungen und Wirrungen ist, zeigt eine aktuelle Untersuchung zu sog. „medizinischen Kehrtwendungen“ [198]. Ein kritischer Umgang mit Innovationen und ein Abwarten, bis sich erste Evidenz für oder gegen einen Nutzen ergibt, erscheint daher im Allgemeinen sinnvoll zu sein.

\subsubsection{EBM versus Real World Data und Big Data}

Die Übertragbarkeit von Erkenntnissen der evidenzbasierten Medizin (EBM) aus randomisierten, kontrollierten Studien (RCT) auf die „Real World“ muss unter Berücksichtigung der Tatsache erfolgen, dass es möglicherweise Unterschiede zwischen einer streng selektierten und homogenen Studienpopulation und der Anwendungspopulation gibt [199]. Diese sog. Extrapolation von EBM ist derzeit Gegenstand intensiver Diskussion [200]. Eine Relativierung der Bedeutung von EBM und RCT erfolgt nicht nur durch Befürworter der Präferenz von „Real World Data“ [201], sondern auch im Kontext von beschleunigten Arzneimittelzulassungen als sog. „Adaptive Pathways“, bei denen Anwendungsbeobachtungen ein gröBeres Gewicht als RCTs erhalten sollen [202]. 
Auch von Seiten „Big Data“ werden die über die letzten Jahrzehnte entwickelten wissenschaftlichen Qualitätsstandards zum Bewerten von Kausalität in Frage gestellt: Kausalität wird bei „Big Data“ durch Korrelation ersetzt [201]. Mit der entsprechenden Vorsicht sind deshalb die Ergebnisse zu interpretieren.

\subsubsection{Evidenzbasierte Chirurgie}

Neue chirurgische Verfahren werden, oft in Zusammenhang mit medizintechnischem Fortschritt, von wenigen Chirurgen entwickelt, von ihnen am eigenen Patientengut erprobt und dann mit dem wahrgenommenen Mehrwert präsentiert. Anders als bei nicht-chirurgischen Interventionen sind Placebo-kontrollierte RCTs für chirurgische Interventionen selten und ungewöhnlich. Es wird kontrovers diskutiert, ob es ethisch vertretbar ist, einen Patienten der Placebo-Gruppe einer potenziellen Komplikation, dem Narkoserisiko und der Hautnarbe der Scheinoperation auszusetzen.

Das Ergebnis eines chirurgischen Eingriffs hängt von 3 Faktoren ab: dem ausschlaggebenden Element der chirurgischen Prozedur, dem Placebo-Effekt und unspezifischen Effekten. Der Placebo-Effekt ist bei chirurgischen Interventionen wegen des suggestiven Settings eher hoch anzusetzen.

Insbesondere bei elektiven Eingriffen zur Verbesserung der Lebensqualität, deren Mehrwert gegenüber einem konservativen Vorgehen in Fachkreisen als fraglich angesehen wird, werden Placebo-kontrollierte RCTs als ethisch gerechtfertigt angesehen [203]. Eine aktuelle Übersicht hat 53 Studien ausgewertet. Bei knapp der Hälfte der Studien konnte kein signifikanter Nutzen im Vergleich zum Placebo-Arm festgestellt werden. Bei den Studien mit überlegenem Verum-Arm war auch im Placebo-Arm ein kleinerer Nutzen zu messen [204]. Die bekannteste Studie auf unserem Fachgebiet ist die von Thomsen, der für die Dekompression des Saccus endolymphaticus bei M. Ménière schon 1981 zeigen konnte, dass Operation und Scheinoperation zu gleich guten, über eine Nachbeobachtungszeit von 3 Jahren stabilen Ergebnissen führen und diese daher als reiner Placebo-Effekt angesehen werden müssen [205, 206].

So ungewohnt der Gedanke an evidenzbasierte Chirurgie auch sein mag, es macht sicher Sinn, darüber nachzudenken, welchen Anteil der Placebo-Effekt am PRO (patient reported outcome) unserer operativen Therapien hat - und ob man nicht anstatt einer Saccus-Dekompression nur einen retroaurikulären Hautschnitt durchführen sollte.

Weniger kontrovers und äußerst sinnvoll ist der Vergleich zweier chirurgischer Verfahren mit gleichem Ziel in Form einer RCT. Als Beispiel wäre hier die geplante Studie zum Vergleich von Tonsillektomie und Tonsillotomie zu nennen (s. 5.2.8.2).

\subsection{Qualitätsmanagement: Finanzierung überfällig - mit begleitender RCT}

Die Tatsache, dass 2/3 aller deutschen Krankenhäuser bzw. $23 \%$ aller HNO-Klinken und $87 \%$ aller Praxen bzw. 76 \% aller HNO-Praxen nicht über ein nachgewiesenes, internes Qualitätsmanagementsystems verfügen, steht leider nicht im Vordergrund der aktuellen gesundheitspolitischen Diskussion.

Das Betreiben eines Qualitätsmanagementsystems verursacht Kosten [207], die unter den allgemeinen Bedingungen der Ökonomisierung und der Unterfinanzierung offenbar mehrheitlich eingespart werden. Der rasante Zuwachs der Beteiligung an der IQM, die weitgehend kostenneutral ist, deutet auch darauf hin. Die IQM ist allerdings ein reduzierter Ansatz und bewertet nicht Vorhandensein und Konformität eines internen Qualitätsmanagementsystems (s. 6.10.4).

Analog zum SVR-Vorschlag zur Finanzierung der Weiterbildung ist die Einführung eines Qualitätsfonds zu fordern, aus dem die Betriebskosten eines nachgewiesenen internen Qualitätsmanagementsystems erstattet werden. Krankenhäuser und Praxen sollten im Gegenzug nachweisen, dass sie über ein internes Qualitätsmanagementsystem verfügen (wozu sie seit 15 Jahren gesetzlich verpflichtet sind).

Möglich wäre das über die Teilnahme an einem Zertifizierungsverfahren wie KTQ oder ISO, die über bewährte Verfahren zur Prüfung des Vorhandenseins und ordnungsgemäßen Betreibens eines Qualitätsmanagementsystems verfügen. Alternative Nachweisverfahren über eine Erweiterung der externen Qualitätssicherung sind denkbar. Die ggf. anfallenden Kosten für eine Zertifizierung müssten ebenfalls erstattet werden.

Es würde sich anbieten, beim Umsetzen dieser vermutlich mehrjährigen Maßnahme die Gelegenheit wahrzunehmen, den Nutzen eines Qualitätsmanagementsystems und einer Zertifizierung durch eine RCT zu begleiten, da entsprechende aussagekräftige Untersuchungen weltweit bisher nicht vorliegen (s. 4.8). Mit Studiendesign und Durchführung könnte das IQTIG beauftragt werden.

\subsection{Externe Qualitätssicherung}

Um valide Aussagen über die Behandlungsqualität treffen zu können, sind zusätzlich zu prozessbezogenen Qualitätsindikatoren auch sektorenübergreifende Nachverfolgung und PRO notwendig. Beides wird derzeit entwickelt oder initial implementiert. Eine Betrachtung der Qualität der Gesundheitsorganisationen oberhalb der Prozessebene, d. h. bez. der Implementation eines Qualitätsmanagementsystems, erscheint ebenfalls sinnvoll (ausführlich diskutiert in 5.12).

\subsection{Diskussion - komplexe Herausforderungen}

Die Herausforderungen an eine qualitativ hochwertige Versorgung bestehen in vieler Hinsicht:

Ökonomisierung, Fehlanreize und Unterfinanzierung v. a. der Maximalversorger und Universitätskliniken führen zu Unter-, Über- und Fehlversorgung der Patienten und zu so erheblichen Belastungen der Mitarbeiter, dass deren Ausbildung und Gesundheit Schaden nehmen können und Personalmangel besteht. Die Infrastruktur der Krankenhäuser ist veraltet, v. a. mit Blick auf die Digitalisierung. Deutliche Korrekturen im System der Finanzierung von Betriebs- und Investitionskosten sind dringend erforderlich.

Unter dem Primat der Versorgungsgerechtigkeit und des Patientenwohls sollte eine sektorenübergreifende Anpassung der ambulanten und stationären Versorgungsstrukturen unter Einbeziehung aller Beteiligten der gemeinsamen Selbstverwaltung erfolgen, um die knappen Ressourcen optimal zum Wohl aller einzusetzen. Als erstes sollte dabei die Notfallversorgung reorganisiert werden.

Wachsende legitime Erwartungen von Patienten an Partizipation, aber auch nicht legitime Anspruchshaltungen stellen erhöhte Anforderungen an die Kommunikation.

Der rasante wissenschaftliche und technologische Fortschritt stellt bewährte Konzepte der Evidenzgewinnung in Frage. Die me- 
dizinisch-wissenschaftliche Gemeinschaft wird Antworten zu Fragen finden müssen, die von Big Data, Personalisierung und Individualisierung der Medizin aufgeworfen werden.

Die Implementation von einrichtungsinternem Qualitätsmanagement hat nachvollziehbar unter der Einführung des DRGSystems gelitten und ist bis heute, obwohl gesetzlich verpflichtend, nicht flächendeckend erfolgt. Die notwendigen Ressourcen sollten als Teil der Betriebskosten refinanziert werden. In der externen Qualitätssicherung sind weitere Anstrengungen erforderlich, valide ergebnisbezogene Qualitätsmessungen zu entwickeln.

\section{Ausblick - und zunächst ein Rückblick}

Ein Gedanke des Qualitätsmanagements ist, dass Menschen die höchste innere Befriedigung an ihrer Arbeit finden, wenn es ihnen gelingt, sie selbstbestimmt und besonders gut zu leisten, und dass jeder einen feinen Sinn dafür hat, ob dies gegeben ist oder nicht. Das gilt im Besonderen für unseren Beruf, weil wir unsere Arbeit zum Nutzen von erkrankten, leidenden Mitmenschen leisten. Die Hinwendung zum Menschen ist die Essenz der ärztlichen Grundmotivation

\subsection{Blick zurück}

Eine interessante Perspektive ergibt sich beim Blick zurück auf ein 20 Jahre altes Positionspapier von BÄK und KBV zur Qualitätssicherung: Es wurde u. a. die sektorenübergreifende Zusammenarbeit mit einheitlicher Qualitätsbetrachtung, die Validierung von Qualitätsindikatoren und die Evaluierung von Qualitätssicherungsmaßnahmen, das Primat von beratenden über reglementierende Qualitätssicherungsverfahren und Patientenbefragungen im Sinne von PRO gefordert. Alle Punkte sind heute noch aktuell, wenig davon wurde umgesetzt. Die wichtigste Forderung wird aus einem Beschluss des 96. Ärztetags von 1993 (!) zitiert:

\section{„Qualitätssicherung bedarf angemessener personeller und organisatorischer Strukturen. Diese sind mit Kosten verbunden. Für den finanziellen Mehraufwand, der den Teilnehmern an Qualitätssicherungsmaßnahmen entsteht, sind zusätzlich notwendige Finanzierungsmittel bereitzustellen “ [208].}

Wie bekannt, wurde auch diese Forderung nicht erfüllt. Stattdessen setzte mit der DRG-Einführung erschwerend die Ökonomisierung ein mit den beschriebenen Folgen für eine evidenzbasierte, patientenorientierte Medizin. Das von der gemeinsamen Selbstverwaltung ins Leben gerufene KTQ-Projekt überschritt 2009 seinen Zenit und ist seitdem auf Talfahrt. Zwei Drittel aller Krankenhäuser verfügen über kein nachgewiesenes Qualitätsmanagementsystem.

\subsection{Politik und Qualität}

Sachverständige Analysen über gegenwärtige Fehlentwicklungen und Konzepte für eine qualitätsorientierte Weiterentwicklung des Gesundheitswesens liegen in ausgezeichneter Güte und Klarheit vor und stimmen weitgehend überein. Es liegt jetzt an den politischen Akteuren der Bundes- und Landespolitik und der gemeinsamen Selbstverwaltung, in welche Richtung sich das Gesundheitswesen in Zukunft bewegen wird:
- Weiter auf den Weg der Ökonomisierung mit Fehlanreizen, Unterfinanzierung der Spitzenmedizin, Zentralisierung (bzw. Rationierung) der Krankenhausleistungen á la Bertelsmann, Personalmangel, einem Qualitätsmanagement, das nur Mindestanforderungen erfüllt und deshalb durch eine externe Qualitätssicherung mit verschärfter Kontrolle und Sanktionen durchgesetzt werden muss, flankiert von dirigistischen Eingriffen in die Autonomie der Leistungserbringer.

- Oder zu einer qualitätsorientierten, patienten- und mitarbeiterfokussierten Medizin, indem die Ökonomisierung des Gesundheitswesens eingehegt wird, Fehlanreize korrigiert werden und eine adäquate, sektorenübergreifende Kapazitätsplanung zur angemessenen medizinischen Versorgung der Bevölkerung umgesetzt wird. So könnten u. a. auch die Ressourcen freigesetzt werden, die für die überfällige Refinanzierung eines einrichtungsinternen Qualitätsmanagements notwendig sind.

\subsection{Entwicklungen im Qualitätsmanagement}

Die generelle ärztliche Bereitschaft, jenseits der verpflichtenden externen Qualitätssicherung aktiv zu werden, zeigt sich in der starken Beteiligung an zahlreichen freiwilligen Qualitätsinitiativen und der IQM. Durch letztere gewinnt auch die kollegiale Beratung durch das ärztliche Peer-Review der BÄK weiter an Verbreitung.

Die absehbare Entwicklung, verstärkt Ergebnisqualität und PRO in die externe Qualitätssicherung einzubeziehen, wird einen starken Anreiz an die Leistungserbringer zur Selbstoptimierung mittels eines umfassenden Qualitätsmanagementsystems setzen. Es wird sich zeigen, ob dieses ausschließlich intern angewendet werden wird, oder ob die Zertifizierungen bzw. Akkreditierungen wieder zunehmen bzw. die vom G-BA betriebene Qualitätssicherung zur Akkreditierung ausgebaut werden wird, wie von der AWMF angeregt. Es wäre wünschenswert, dass damit der Exzellenzgedanke und eine wiedererstarkte Qualitätskultur mehr in den Vordergrund rücken.

Die HNO-Heilkunde unterliegt derzeit nur im vertragsärztlichen Bereich der externen Qualitätssicherung - zukünftig ist eine Einbeziehung des Krankenhausbereichs zu erwarten.

\subsection{Technologie}

Die Informationstechnologie (IT) wird nicht nur große Chancen bieten, Behandlungsfehler zu verringern, sondern auch im Zusammenspiel mit Fortschritten in der Biotechnologie durch die Personalisierung von Prävention und Therapie ein qualitativ deutlich höheres Qualitätsniveau zu erreichen (s. 8.3). Die dazu notwendige Digitalisierung der Krankenhäuser muss entschieden vorangetrieben werden.

Als eine interessante Anwendung zeichnet sich z. B. die KI-gestützte, automatisierte Fehleranalyse von endoskopischen OP-Videos ab. Die IT bietet auch unmittelbar für das Qualitätsmanagement neue und elegante Möglichkeiten zur Ablaufoptimierung, z. B. durch Simulation von Prozessen, unterstützt durch MotionTracking von Patienten zum Erfassen des Ist-Zustandes [209].

Es werden kontroverse, anstrengende politische Entscheidungen über den Umgang mit den hochsensiblen Gesundheitsdaten notwendig werden, um die Interessen aller an den oben skizzierten Entwicklungen sicherzustellen, aber einen Missbrauch mit dem Potenzial zur sozialen Dystopie zu verhindern. Beispielweise seien hier Meldungen 
über Algorithmen oder KI genannt, die das wahrscheinliche Eintreten des Todesfalls von Patienten innerhalb eines Jahres vorhersagen sollen können, um Patienten rechtzeitig der Palliation zuzuleiten und „fehlgeleitete“ Ausgaben einzusparen [210, 211].

\section{5 Ärzte und Mitarbeiter}

Trotz aller beschriebenen Einschränkungen und Herausforderungen wird durch das Verantwortungsbewusstsein und die hohe Leistungsbereitschaft der Ärzte und aller mit uns zusammenarbeitenden Berufsgruppen eine insgesamt sehr gute Versorgung der uns anvertrauten Patienten erreicht. Dabei wird jedoch oft die eigene Belastungsgrenze überschritten mit den zuvor beschriebenen Folgen für Gesundheit, Qualität der Arbeit und Personalstand. Wenn nicht rasch durch Verbesserung der Arbeitsbedingungen das ärztliche und pflegerische Berufsbild wieder attraktiver werden, ist mit gravierenden Auswirkungen auf die Qualität der Versorgung zu rechnen.

Die ärztliche Stimme muss hörbar werden im politischen Hintergrundrauschen, um mit unserem Sachverstand zur notwendigen und überfälligen Wende in der Gesundheitspolitik beizutragen.

\section{Interessenkonflikt}

Der Autor gibt an, dass kein Interessenkonflikt besteht.

\section{Literatur}

[1] Brennan TA, Leape LL, Laird NM et al. Incidence of Adverse Events and Negligence in Hospitalized Patients. New England Journal of Medicine 1991; 324: 370-376

[2] Unerwünschte Ereignisse https://www.aezq.de/patientensicherheit/ unerwuenschte-Ereignisse, aufgerufen am 11.08.2019

[3] Agenda Patientensicherheit. 2007; https://www.aps-ev.de/ wp-content/uploads/2016/10/Agenda_2007_mit_Titelblatt.pdf, aufgerufen am 11.08.2019

[4] Reason J. Understanding adverse events: human factors. Qual Health Care 1995; 4: 80-89

[5] Reason J. Human error: models and management. BM] (Clinical research ed). 2000: 320: 768-770

[6] Smith CM. Origin and uses of primum non nocere - above all, do no harm!. J Clin Pharmacol 2005; 45: 371-377

[7] Guo Z. Chinese Confucian culture and the medical ethical tradition. J Med Ethics 1995; 21: 239-246

[8] Genfer Deklaration https://www.bundesaerztekammer.de/fileadmin/ user_upload/downloads/pdf-Ordner/International/Deklaration_von_ Genf_DE_2017.pdf, aufgerufen am 10.08.2019

[9] Deklaration von Helsinki https://www.bundesaerztekammer.de/ fileadmin/user_upload/Deklaration_von_Helsinki_2013_DE.pdf, aufgerufen am 10.08.2019

[10] Donabedian A. Evaluating the quality of medical care. Milbank Mem Fund Q 1966; 44 (Suppl): 166-206

[11] Institute. of Medicine. Committee to Design a Strategy for Quality Review and Assurance in Medicare. In: KN L, Hrsg. Medicare: A Strategy for Quality Assurance Volume II: Sources and Methods. Washington, DC: Institute of Medicine; 1990

[12] GMDS. Arbeitsgruppe „Qualitätsmanagment in der Medizin“ Begriffe und Konzepte des Qualitätsmanagements. In: GMDS, Hrsg. 3. Aufl. Hannover; 2008
[13] Definitionen und Konzepte des Qualitätsmanagements https://www. aezq.de/aezq/kompendium_q-m-a/2-definitionen-und-konzeptedes-qualitaetsmanagements/\#2.1, aufgerufen am 11.08.2019

[14] Methodische Grundlagen v1.1 | IQTIG https://iqtig.org/dateien/ dasiqtig/grundlagen/IQTIG_Methodische-Grundlagen-V1.1_barrierefrei_2019-04-15.pdf, aufgerufen am 16.07.2019

[15] The Deming Society https://deming.org/deming/deming-the-man, aufgerufen am 11.08.2019

[16] Qualitätsindikatoren https://iqtig.org/qs-instrumente/qualitaetsindikatoren/, aufgerufen am 12.08.2019

[17] Koller M, Neugebauer EA, Augustin M et al. Die Erfassung von Lebensqualität in der Versorgungsforschung - konzeptuelle, methodische und strukturelle Voraussetzungen. Gesundheitswesen 2009; 71: 864-872

[18] Lebensqualität https://www.rki.de/DE/Content/Gesundheitsmonitoring/Gesundheitsberichterstattung/GesundAZ/Content/G/Gesbez_Lebensqualitaet/Inhalt/Lebensqualitaet.html, aufgerufen am 11.08.2019

[19] Bulut OC, Wallner F, Oladokun D et al. Long-term quality of life changes after primary septorhinoplasty. Qual Life Res 2018; 27: 987-991

[20] Bulut OC, Wallner F, Plinkert PK et al. Quality of life after septorhinoplasty measured with the Functional Rhinoplasty Outcome Inventory 17 (FROI-17). Rhinology 2015; 53: 54-58

[21] Brauer CM. Champions of Quality in Health Care. Lyme, Connecticut, USA: Greenwich Publishing Group, Inc. 2001

[22] The Joint Commission - Over a century of quality and safety https:// jntcm.ae-admin.com/assets/1/6/TJC_history_timeline_ through_2018.pdf, aufgerufen am 28.07.2019

[23] Donahue KT, vanOstenberg P. Joint Commission International accreditation: relationship to four models of evaluation. Int J Qual Health Care 2000; 12: 243-246

[24] The way to ZERO https://www.jointcommission.org/leadingthewaytozero.aspx, aufgerufen am 28.07.2019

[25] Mehta A, Goldstein SD, Makary MA. Global trends in center accreditation by the Joint Commission International: growing patient implications for international medical and surgical care. J Travel Med 2017; 24:

[26] JCl-Accredited Organizations https://www.jointcommissioninternational.org/about-jci/jci-accredited-organizations/?c= Germany\& $\mathrm{a}=$ Hospital \%20Program, aufgerufen am 22.07.2019

[27] The ISO Story https://www.iso.org/the-iso-story.html\#2, aufgerufen am 29.07.2019

[28] Steinbrucker S. Qualitätsmanagementsysteme sind Pflichtprogramm: die Kliniken haben die Wahl. Ein Vergleich der QM-Systeme DIN EN ISO 9000 ff, KTQ(R) und EFQM. Radiologe 2011; 51: 835-843

[29] ISO 9001:2015(en) Quality management systems - Requirements https://www.iso.org/obp/ui/\#iso:std:iso:9001:ed-5:v1:en, aufgerufen am 29.07.2019

[30] Welche Aufgaben hat die DAkkS? https://www.dakks.de/content/ welche-aufgabe-hat-die-dakks, aufgerufen am 30.07.2019

[31] Neuerliche Verteuerung der Akkreditierung nicht zumutbar, nicht hinnehmbar und nicht vermittelbar! https://vup.de/artikel. php?typ = i\&id = 2518, aufgerufen am 30.07.2019

[32] Lähmungen im Akkreditierungssystem beseitigen! VUP-Geschäftsführer vor Wirtschaftsausschuss des Bundestages https://vup.de/ artikel. html?typ = i\&id = 2794, aufgerufen am 30.07.2019

[33] Akkreditierung: Situation und Handlungsbedarf https://vup.de/ dokumente_14/2737_1808\%20Hintergrund\&Handlungsbedarf\%20 Akkreditierungswesen.pdf, aufgerufen am 30.07.2019

[34] ISO 9001:2015 und DIN EN 15224 - Ist KTQ aus dem Rennen? https://jomec.de/beitrag/news/detail/News/iso-90012015-und-dinen-15224-ist-ktq-aus-dem-rennen/, aufgerufen am 29.07.2019 
[35] Das KTQ-Verfahren https://ktq.de/index.php?id=9, aufgerufen am 21.07.2019

[36] Ablauf des KTQ-Verfahrens Version 1.4 https://www.ktq.de/ fileadmin/media/Dokumente\%20Bereiche/Verfahrensablauf_KTQ_ Verfahren_1_4.pdf, aufgerufen am 22.07.2019

[37] KTQ-Plus-Verfahren https://www.ktq.de/index.php?id=929, aufgerufen am 22.07.2019

[38] KTQ Best-Practice https://www.ktq.de/index.php?id=607, aufgerufen am 22.07.2019

[39] KTQ-Qualitätsmanagement-Verfahren aktualisiert und weiterentwickelt https://www.aerzteblatt.de/nachrichten/63298/KTQ-Qualitaetsmanagement-Verfahren-aktualisiert-und-weiterentwickelt, aufgerufen am 21.07.2019

[40] KTQ-Newsletter 1/2019 https://www.ktq.de/index.php?id=999, aufgerufen am 22.07.2019

[41] KTQ-Newsletter 2/2019 https://www.ktq.de/index.php?id = 1012, aufgerufen am 22.07.2019

[42] Kooperation für Transparenz und Kooperation im Gesundheitswesen https://de.wikipedia.org/wiki/Kooperation_für_Transparenz_und_ Qualität_im_Gesundheitswesen\#Relevanz_von_KTQ_im_deutschen_ Gesundheitswesen, aufgerufen am 22.07.2019

[43] pCC inkl. KTG-KH https://www.procum-cert.de/dienstleistungen/ ueberblick/pcc-inkl-ktq-kh/, aufgerufen am 22.07.2019

[44] KTQ-Zertifikate in den verschiedenen Bereichen https://ktq.de/index. php?id = 169, aufgerufen am 21.07.2019

[45] Our History https://www.efqm.org/index.php/about-us/our-history/, aufgerufen am 24.07.2019

[46] Ludwig-Erhard-Preis https://ilep.de/, aufgerufen am 26.07.2019

[47] What is the history of EFQM? https://www.efqm.org/index.php/ knowledge-base/what-is-the-history-of-efqm/, aufgerufen am 24.07.2019

[48] EFQM. Das EFQM Excellence Modell 2013. 2012

[49] EFQM Recognition https://www.efqm.org/index.php/efqm-recognition/ aufgerufen am 26.07.2019

[50] Longmuir $\mathrm{R}$. Where are we at EFQM and more importantly where are we in the EFQM model 2020 developement? EFQM Magazine I Design for Excellence June 2019

[51] Jarosz C. Digital Disruption EFQM Magazine | Design for Excellence. June 2019;

[52] Where are we at EFQM and more importantly where are we in the EFQM Model 2020 development? https://www.youtube.com/ watch?v = vklzgaBhMMs aufgerufen am 23.07.2019

[53] Recognition Database | Germany | Healthcare Services http://www. shop.efqm.org/recognition-database/ aufgerufen am 19.07.2019

[54] KTQ-Kooperation für Transparenz und Qualität im Krankenhaus https://www.krankenhausberater.de/fileadmin/Assets/publikation/ pflegebulletin-ktq-verfahren-ktq-katalog-ktq-modell-stefan-ruhlruediger-herbold-bernd-schlieder.pdf, aufgerufen am 21.07.2019

[55] KBV - QEP - Qualität und Entwicklung in Praxen https://www.kbv.de/ html/qep.php, aufgerufen am 30.07.2019

[56] Gibis B, Diel F. QEP - Qualität und Entwicklung in Praxen: Systematisch zum Erfolg. Dtsch Arztebl International 2005; 102: 16

[57] Qualitätsmanagment KPQM KVWL https://www.kvwl.de/arzt/qsqm/ management/index.htm aufgerufen am 30.07.2019

[58] QM für die Arztpraxis mit EPA https://www.epa-qm.de/startseite/ index.html aufgerufen am 30.07.2019

[59] QM-Verfahren im Vergleich | KBV https://www.kbv.de/media/sp/ QM_Verfahren_im_Vergleich.pdf, aufgerufen am 18.07.2019

[60] Gumpert M, Reese JP. Analyse einzelner Qualitätskategorien aus Qualitätsmanagementsystemen und deren Einfluss auf das Gesamtsystem. Z Evid Fortbild Qual Gesundhwes 2018; 139: 28-36
[61] Der Zentrumsbegriff in der Medizin https://www.bundesaerztekammer.de/aerzte/qualitaetssicherung/zentren-und-zertifizierung/ zentrumsbegriff/ aufgerufen am 30.07.2019

[62] Das Zentrenmodell https://www.krebsgesellschaft.de/deutschekrebsgesellschaft-wtrl/deutsche-krebsgesellschaft/zertifizierung/ das-zentrenmodel-der-dkg.html aufgerufen am 30.07.2019

[63] Qualitätsmerkmale eines krankheitsorientierten Zentrums https:// www.bundesaerztekammer.de/fileadmin/user_upload/downloads/ Qualitaetsmerkmale.pdf, aufgerufen am 30.07.2019

[64] Zentren und Zertifizierung https://www.bundesaerztekammer.de/ aerzte/qualitaetssicherung/zentren-und-zertifizierung/ aufgerufen am 30.07.2019

[65] Zertifizierung im medizinischen Kontext https://www.bundesaerztekammer.de/fileadmin/user_upload/downloads/Zertifizierung_im_ medizinischen_Kontext.pdf, aufgerufen am 30.07.2019

[66] Flodgren G, Goncalves-Bradley DC, Pomey MP. External inspection of compliance with standards for improved healthcare outcomes. Cochrane Database Syst Rev 2016; 12: Cd008992

[67] Flodgren G, Pomey MP, Taber SA et al. Effectiveness of external inspection of compliance with standards in improving healthcare organisation behaviour, healthcare professional behaviour or patient outcomes. Cochrane Database Syst Rev 2011; doi:10.1002/14651858.CD008992.pub2 Cd008992

[68] Lam MB, Figueroa JF, Feyman Y et al. Association between patient outcomes and accreditation in US hospitals: observational study. BM] 2018; 363: k4011

[69] Sack C, Scherag A, Lutkes P et al. Is there an association between hospital accreditation and patient satisfaction with hospital care? A survey of 37,000 patients treated by 73 hospitals. Int J Qual Health Care 2011; 23: 278-283

[70] Einfluß der Zentrenbildung auf die Versorgung https://www. bundesaerztekammer.de/fileadmin/user_upload/downloads/ Einfluss_der_Zentrenbildung_auf_die_Versorgung.pdf, aufgerufen am 30.07.2019

[71] Griffiths A, Leaver MP. Wisdom of patients: predicting the quality of care using aggregated patient feedback. BMJ Qual Saf 2018; 27: 110-118

[72] Neugebauer F, Schiller M, Luger TA et al. Evaluation einrichtungsinterner Effekte nach Durchführung eines Zertifizierungsverfahrens im Krankenhaus. Gesundheitswesen 2013; 75: e108-e112

[73] Berssaneti FT, Saut AM, Barakat MF et al. Is there any link between accreditation programs and the models of organizational excellence? Rev Esc Enferm USP 2016; 50: 650-657

[74] Gesetz zur Modernisierung der gesetzlichen Krankenversicherung(GKV-Modernisierungsgesetz - GMG) https:// www.bgbl.de/xaver/bgbl/start.xav?startbk= Bundesanzeiger_ BGBI\&jumpTo = bgbl103s2190.pdf, aufgerufen am 06.07.2019

[75] Arzneimittel-Nutzenbewertungsverordnung - AM-NutzenV §35a Absatz 1 SGB V http://www.gesetze-im-internet.de/am-nutzenv/_4. html aufgerufen am 06.07.2019

[76] Patientenrechtegesetz https://www.bundesgesundheitsministerium. de/service/begriffe-von-a-z/p/patientenrechtegesetz.html aufgerufen am 04.08.2019

[77] Sicherstellungszuschläge für 120 ländliche Krankenhäuser https:/| www.aerzteblatt.de/nachrichten/104458/Sicherstellungszuschlaegefuer-120-laendliche-Krankenhaeuser aufgerufen am 13.08.2019

[78] Mühlbauer V, Teupen S. Gemeinsamer Bundesausschuss und Institut für Qualität und Wirtschaftlichkeit im Gesundheitswesen. Aufgaben und Struktur unter Berücksichtigung der Patientenbeteiligung. Dtsch Med Wochenschr 2014; 139: 147-151

[79] Wer wir sind - Gemeinsamer Bundesausschuss https://www.g-ba.de/ ueber-den-gba/wer-wir-sind/ aufgerufen am 08.07.2019 
[80] Qualitätssicherung im Krankenhaus | BMG https://www.bundesgesundheitsministerium.de/qualitaet-krankenhausversorgung.html aufgerufen am 01.07.2019

[81] Finanzierung des G-BA https://www.g-ba.de/ueber-den-gba/ finanzierung/systemzuschlaege/ aufgerufen am 14.07.2019

[82] Qualitätssicherung - Gemeinsamer Bundesausschuss https:// www.g-ba.de/themen/qualitaetssicherung/ aufgerufen am 09.07.2019

[83] Qualitätsmanagement-Richtlinie/QM-RL - G-BA https://www.g-ba. de/downloads/62-492-1296/QM-RL_2015-12-17_iK-2016-11-16.pdf, aufgerufen am 09.07.2019

[84] Neufassung der Regelungen zur Fortbildung im Krankenhaus: Harmonisierung mit den vertragsärztlichen und berufsrechtlichen Regelungen zur Fortbildungspflicht - G-BA https://www.g-ba.de/ downloads/39-261-1589/2012-10-18_FKH-R_Neufassung_BAnz.pdf, aufgerufen am 10.07.2019

[85] CIRS medical Anästesiologie https://www.cirs-ains.de/, aufgerufen am 10.07.2019

[86] Netzwerk CIRS Berlin https://www.cirs-berlin.de/, aufgerufen am 10.07.2019

[87] Krankenhaus-CIRS-Netz Deutschland 2.0 https://www.kh-cirs.de/ index.html aufgerufen am 10.07.2019

[88] Regelungen zum Qualitätsbericht der Krankenhäuser, Qb-R https:// www.g-ba.de/downloads/62-492-1789/Qb_R_2018-12-20_iK-201903-12.pdf, aufgerufen am 10.07.2019

[89] Referenzdatenbank - Die Qualitätsberichte der Krankenhäuser https://g-ba-qualitaetsberichte.de/\#/search aufgerufen am 10.07.2019

[90] Berichterstattung zur Umsetzung von Qualitätsmanagement in der vertragszahnärztlichen Versorgung https://www.g-ba.de/downloads/17-98-4729/2018-11-22_Bericht-2017-KZBV.pdf, aufgerufen am 15.07.2019

[91] Bericht zur Umsetzung von Qualitätsmanagement in Vertragsarztpraxen und Medizinischen Versorgungszentren https://www.g-ba.de/ downloads/17-98-4728/2018-11-22_Bericht-2017-KBV.pdf, aufgerufen am 15.07.2019

[92] Richtlinie des Gemeinsamen Bundesausschusses zur datengestützten einrichtungsübergreifenden Qualitätssicherung https://www.g-ba. de/downloads/62-492-1865/DeQS-RL_2019-05-16_iK-2019-07-05_ AT-04-07-2019-B3.pdf, aufgerufen am 10.07.2019

[93] Pressemitteilung: Neues datengestütztes Qualitätssicherungsverfahren zu Nierenersatztherapien beschlossen - Behandlungsdaten werden zukünftig sektorenübergreifend erhoben https://www.g-ba. de/downloads/34-215-801/18_2019-06-20_QS-NET.pdf, aufgerufen am 11.07.2019

[94] Richtlinie über Maßnahmen der Qualitätssicherung in Krankenhäusern/QSKH-RL https://www.g-ba.de/downloads/62-492-1834/ QSKH-RL_2019-03-22_iK-2019-06-01.pdf, aufgerufen am 11.07.2019

[95] Die externe stationäre Qualitätssicherung in den Landesgeschäftsstellen für Qualitätssicherung (LQS) https://www.dkgev.de/fileadmin/ default/Mediapool/2_Themen/2.6._Qualitaet_Hygiene_und_Sicherheit/2.6.2._Stationaere_und_sektorenuebergreifende_datengestuetze_Qualitaetssicherung/DKG_LQS_Broschur_2015.pdf, aufgerufen am 11.07.2019

[96] Qualitätsreport 2017 | IQTIG https://iqtig.org/downloads/ berichte/2017/IQTIG_Qualitaetsreport-2017_2018_09_21.pdf, aufgerufen am 12.07.2019

[97] Qualitätssicherungs-Richtlinie Dialyse / QSD-RL https://www.g-ba. de/downloads/62-492-1379/QSD-RL_2017-01-19_iK-2017-04-12_ AT-11-04-2017-B3.pdf, aufgerufen am 14.07.2019

[98] Planungsrelevante Qualitätsindikatoren: Länder ziehen nicht mit https://www.aerzteblatt.de/archiv/202399/Planungsrelevante-Qualitaetsindikatoren-Laender-ziehen-nicht-mit, aufgerufen am 21.07.2019
[99] Richtlinie zu planungsrelevanten Qualitätsindikatoren - planQI-RL https://www.g-ba.de/downloads/62-492-1826/plan-QIRL_2018-12-20_iK_2019-05-23.pdf, aufgerufen am 15.07.2019

[100] Richtlinie zu planungsrelevanten Qualitätsindikatoren. Veröffentlichung des Berichts. 2017; https://www.g-ba.de/beschluesse/3545/, aufgerufen am 15.07.2019

[101] Neue Qualitätsindikatoren zeigen Wirkung https://www.aerzteblatt. de/nachrichten/103025/Neue-Qualitaetsindikatoren-zeigen-Wirkung, aufgerufen am 21.07.2019

[102] Stellungnahme der Arbeitsgemeinschaft der Wissenschaftlichen Medizinischen Fachgesellschaften (AWMF) zu „Methodische Grundlagen V1.0s - Entwurf für das Stellungnahmeverfahren“ vom 15.11.2018 des Instituts für Qualität und Transparenz im Gesundheitswesen (IQTIG) https://www.awmf.org/fileadmin/user_upload/ Stellungnahmen/Medizinische_Versorgung/20190115_Stellungnahme_AWMF_IQTIG_Methoden1.1_fin.pdf, aufgerufen am 16.07.2019

[103] QP-RL: Aussetzung der Stichprobenprüfungen für das zweite Quartal 2019 https://www.g-ba.de/downloads/40-268-5594/2019-02-21_ QP-RL_Aussetzung-Stichprobenprüfung-2019_TrG.pdf, aufgerufen am 15.07.2019

[104] MDK-Qualitätskontroll-Richtlinie MDK-QK-RL https://www.g-ba.de/ downloads/62-492-1590/MDK-QK-RL_2017-12-21_iK_2018-12-13. pdf, aufgerufen am 15.07.2019

[105] Qualitätsförderungs- und Durchsetzungs-Richtlinie QFD-R https:// www.g-ba.de/downloads/39-261-3766/2019-04-18_QFD-RL_Erstfassung_QFD-RL.pdf, aufgerufen am 15.07.2019

[106] G-BA legt vier Leistungsbereiche für die Erprobung von Qualitätsverträgen fest https://www.g-ba.de/presse/pressemitteilungen/688/, aufgerufen am 15.07.2019

[107] Lohrberg D, Augustin M, Blome C. The definition and role of quality of life in Germany's early assessment of drug benefit: a qualitative approach. Qual Life Res 2016; 25: 447-455

[108] Frühe Nutzenbewertung neuer Arzneimittel. 2019 Gerecht und nachhaltig? https://www.awmf.org/fileadmin/user_upload/ Stellungnahmen/Medizinische_Versorgung/201905_Fruehe_Nutzenbewertung_Positionspapier_1.0.pdf. aufgerufen am 19.07.2019

[109] Wieseler B, McGauran N, Kaiser T. New drugs: where did we go wrong and what can we do better? BM] 2019; 366: 14340

[110] Richtlinie zur Erprobung der Tonsillotomie bei rezidivierender akuter Tonsillitis (ErpRL Tonsillotomie) https://www.g-ba.de/downloads/39-261-3492/2018-09-20_Erp-RL_Tonsillotomie-rezidivierende-akuter-Tonsillitis_BAnz.pdf, aufgerufen am 16.07.2019

[111] §137a SGB V Institut für Qualität und Transparenz im Gesundheitswesen http://www.gesetze-im-internet.de/sgb_5/_137a.html, aufgerufen am 16.07.2019

[112] Sektorenübergreifende Qualität im Gesundheitswesen | AQUAInstitut https://sqg.de, aufgerufen am 16.07.2019

[113] Das IQTIG https://iqtig.org/das-iqtig/, aufgerufen am 16.07.2019

[114] Methodische Grundlagen v1.0 | IQTIG https://iqtig.org/dateien/ dasiqtig/grundlagen/IQTIG_Methodische-Grundlagen-V1.0.pdf, aufgerufen am 16.07.2019

[115] Methodische Grundlagen v1.0s Stellungnahmen | IQTIG https://iqtig. org/dateien/dasiqtig/grundlagen/IQTIG_Methodische-Grundlagen-V1-0s_Stellungnahmen_2018-03-14.pdf, aufgerufen am 16.07.2019

[116] Methodische Grundlagen v1.0s Würdigung der Stellungnahmen | IQTIG https://iqtig.org/dateien/dasiqtig/grundlagen/IQTIG_Methodische-Grundlagen-V1-0s_Wuerdigung-der-Stellungnahmen_2018-03-14.pdf, aufgerufen am 16.07.2019

[117] Methodische Grundlagen v1.1s Würdigung der Stellungnahmen | IQTIG https://iqtig.org/dateien/dasiqtig/grundlagen/IQTIG_Methodische-Grundlagen-V1-1s_Wuerdigung-der-Stellungnahmen_2019-04-15.pdf, aufgerufen am 16.07.2019 
[118] Methodische Grundlagen v1.1s Stellungnahmen | IQTIG https://iqtig. org/dateien/dasiqtig/grundlagen/IQTIG_Methodische-Grundlagen-V11s_Stellungnahmen_2019-04-15.pdf, aufgerufen am 16.07.2019

[119] Tätigkeitsbericht 2018 | IQTIG https://iqtig.org/dateien/berichte/2019/IQTIG_Ta_tigkeitsbericht_2018__WEB-2019-07-15.pdf, aufgerufen am 16.07.2019

[120] Das IQWIG https://www.iqwig.de/, aufgerufen am 17.07.2019

[121] Allgemeine Methoden 5.0 | IQWIG https://www.iqwig.de/download/ Allgemeine-Methoden_Version-5-0.pdf, aufgerufen am 17.07.2019

[122] Die Analyse von „Effizienzgrenzen“: Allgemeine Methoden zur Bewertung von Verhältnissen zwischen Nutzen und Kosten I IQWIG https://www.iqwig.de/download/Allgemeinverstaendliche_Zusammenfassung_Kosten_und_Nutzen_in_der_Medizin.pdf, aufgerufen am 17.07.2019

[123] Bundesärztekammer https://www.bundesaerztekammer.de/ aufgerufen am 17.07.2019

[124] Musterberufsordnung für die in Deutschland tätigen Ärztinnen und Ärzte | BÄK, aufgerufen am 17.07.2019

[125] Aktionsbündnis Patientensicherheit https://www.aps-ev.de/, aufgerufen am 18.07.2019

[126] Aufgaben und Ziele | ÄQZ https://www.aezq.de/aezq/uber/ aufgaben-und-ziele aufgerufen am 17.07.2019

[127] Programm für Nationale Versorgungsleitlinien https://www.leitlinien. $\mathrm{de} / \mathrm{nvl} /$, aufgerufen am 18.07.2019

[128] Qualitätssicherung durch Ärztekammern - Qualitätsbericht von Bundesärztekammer und Landesärztekammern https://www. bundesaerztekammer.de/fileadmin/user_upload/downloads/Kammeruebergreifender_Qualitaetsbericht_2._Auflage.pdf, aufgerufen am 18.07.2019

[129] Netzwerk CIRSmedical.de | ÄZQ https://www.aezq.de/patientensicherheit/cirs/netzwerk-cirsmedical.de aufgerufen am 17.07.2019

[130] AWMF online - Das Portal der wissenschaftlichen Medizin https:// www.awmf.org, aufgerufen am 19.07.2019

[131] AWMF: Qualitätsmanagement https://www.awmf.org/medizinversorgung/qualitaetsmanagement.html aufgerufen am 19.07.2019

[132] Qualitätsbericht 2018 - Berichtsjahr 2017 https://www.kbv.de/ media/sp/KBV_Qualitaetsbericht_2018.pdf, aufgerufen am

[133] Deutsche Krankenhausgesellschaft e.V. https://www.dkgev.de/, aufgerufen am 18.07.2019

[134] Patientenwohl und Daseinsvorsorge POSITIONEN der Deutschen Krankenhausgesellschaft (DKG) für die 19. Legislaturperiode des Deutschen Bundestags https://www.dkgev.de/fileadmin/default/ Mediapool/1_DKG/1.6_Positionen/2017-03_DKG-Positionspapier_19._Legislaturperiode.pdf, aufgerufen am 18.07.2019

[135] GKV-Spitzenverband https://www.gkv-spitzenverband.de, aufgerufen am 18.07.2019

[136] Medizinischer Dienst der Krankenversicherung https://www.mdk.de/, aufgerufen am 18.07.2019

[137] Medizinischer Dienst des Spitzenverbandes Bund der Krankenkassen https://www.mds-ev.de/, aufgerufen am 18.07.2019

[138] Kompetenz-Centrum Qualitätssicherung/Qualitätsmanagement (KCQ) https://www.kcqq.de/de, aufgerufen am 18.07.2019

[139] Die Arbeit der Medizinischen Dienste. Zahlen, Daten, Fakten. 2018; https://www.mdk.de/fileadmin/MDK-zentraler-Ordner/ Downloads/00_MDK_allgemein/2018_MDK_ZDF_Kurzversion.pdf, aufgerufen am 18.07.2019

[140] Sachverständigenrat Gesundheit https://www.svr-gesundheit.de, aufgerufen am 19.07.2019

[141] Gemeinsame Pressemitteilung der Sachverständigenräte Gesundheit und Wirtschaft: Für mehr Strukturwandel in der Krankenhausversorgung https://www.svr-gesundheit.de/index.php?id=643 aufgerufen am 19.07.2019
[142] Kurzfassung des Gutachtens 2018: Bedarfsgerechte Steuerung der Gesundheitsversorgung https://www.svr-gesundheit.de/fileadmin/ user_upload/Gutachten/2018/SVR-Gutachten_2018_Kurzfassung. pdf, aufgerufen am 08.07.2019

[143] Medizin und Ökonomie - Maßnahmen für eine wissenschaftlich begründete, patientenzentrierte und ressourcenbewusste Versorgung https://www.awmf.org/fileadmin/user_upload/Stellungnahmen/Medizinische_Versorgung/20181205_Medizin_ und_\%C3\%96konomie_AWMF_Strategiepapier_V1.0mitLit.pdf, aufgerufen am 08.07.2019

[144] Hintergrundpapier zur qualitätsorientierten Versorgungssteuerung: Wo steht die Qualitätssicherung des G-BA? Welche Konsequenzen ergeben sich für die Ärztekammern? https://www.bundesaerztekammer.de/fileadmin/user_upload/downloads/pdf-Ordner/QS/ Hintergrundpapier_QS.pdf, aufgerufen am 18.07.2019

[145] Stellungnahme der Arbeitsgemeinschaft der Wissenschaftlichen Medizinischen Fachgesellschaften (AWMF) zum Vorbericht des Instituts für Qualität und Transparenz im Gesundheitswesen (IQTIG): „Planungsrelevante Qualitätsindikatoren - Konzept zur Neu- und Weiterentwicklungvom 28.09.2018 https://www.awmf.org/ fileadmin/user_upload/Stellungnahmen/Medizinische_Versorgung/20181109_Stellungnahme_AWMF_PlanQI_Folgeauftrag2_fakt. pdf, aufgerufen am 06.07.2019

[146] Gillam SJ, Siriwardena AN, Steel N. Pay-for-performance in the United Kingdom: impact of the quality and outcomes framework: a systematic review. Ann Fam Med 2012; 10: 461-468

[147] Glickman SW, Ou FS, DeLong ER et al. Pay for performance, quality of care, and outcomes in acute myocardial infarction. Jama 2007; 297: 2373-2380

[148] Langdown C, Peckham S. The use of financial incentives to help improve health outcomes: is the quality and outcomes framework fit for purpose? A systematic review. J Public Health (Oxf) 2014; 36: 251-258

[149] Ryan A, Sutton M, Doran T. Does winning a pay-for-performance bonus improve subsequent quality performance? Evidence from the Hospital Quality Incentive Demonstration. Health Serv Res 2014; 49: 568-587

[150] Nimptsch U, Mansky T. Hospital volume and mortality for 25 types of inpatient treatment in German hospitals: observational study using complete national data from 2009 to 2014. BMJ Open 2017; 7: e016184

[151] Amato L, Colais P, Davoli M et al. Volume and health outcomes: evidence from systematic reviews and from evaluation of Italian hospital data. Epidemiol Prev 2013; 37: 1-100

[152] Spahn will weitere Mindestmengen auf den Weg bringen https:// www.aerzteblatt.de/nachrichten/103020/Spahn-will-weitere-Mindestmengen-auf-den-Weg-bringen aufgerufen am 25.08.2019

[153] Reason J. Beyond the organisational accident: the need for "error wisdom" on the frontline. Qual Saf Health Care 2004; 13 (Suppl 2): ii28-33

[154] Vickers AJ, Bianco F], Serio AM et al. The surgical learning curve for prostate cancer control after radical prostatectomy. J Natl Cancer Inst 2007; 99: 1171-1177

[155] Vickers AJ, Savage C], Hruza M et al. The surgical learning curve for laparoscopic radical prostatectomy: a retrospective cohort study. Lancet Oncol 2009; 10: 475-480

[156] Entscheidungshilfe Mandelentzündung bei Kindern https://www. gesundheitsinformation.de/entscheidungshilfe-mandelentzuendungbei-kindern.3321.de.html, aufgerufen am 04.08.2019

[157] Harter M, Dirmaier J, Scholl I et al. The long way of implementing patient-centered care and shared decision making in Germany. Z Evid Fortbild Qual Gesundhwes 2017; 123-124: 46-51

[158] Ärztliches Peer Review https://www.bundesaerztekammer.de/aerzte/ qualitaetssicherung/aerztliches-peer-review/, aufgerufen am 04.08.2019 
[159] Jeder Fehler zählt https://www.jeder-fehler-zaehlt.de/, aufgerufen am 07.08.2019

[160] Initiative Qualitätsmedizin IQM https://www.initiative-qualitaetsmedizin.de/home/, aufgerufen am 21.07.2019

[161] Nimptsch U, Peschke D, Mansky T. Impact of quality measurement, transparency and peer review on in-hospital mortality - retrospective before-after study with 63 hospitals. Zeitschrift für Evidenz, Fortbildung und Qualität im. Gesundheitswesen 2016; 115: 10-23

[162] ÄQSI Die Datenbank ärztlicher Qualitätssicherungsinitiativen (BÄK) https://www.aeqsi.de/content/hintergrund.php, aufgerufen am 06.07.2019

[163] AWMF Leitlinien https://www.awmf.org/leitlinien.html, aufgerufen am 06.08.2019

[164] Grimshaw JM, Thomas RE, MacLennan G et al. Effectiveness and efficiency of guideline dissemination and implementation strategies. Health Technol Assess 2004; 8: iii-iv 1-72

[165] Thomas L, Cullum N, McColl E et al. Guidelines in professions allied to medicine. Cochrane Database Syst Rev 2000; doi:10.1002/14651858. CD000349: CD000349

[166] Kritische Bewertung medizinischer Leitlinien https://www.aezq.de/ $\mathrm{mdb} / \mathrm{edocs} / \mathrm{pdf} /$ literatur/diss-ml-2009.pdf, aufgerufen am 06.08.2019

[167] Leitlinienprogramm Onkologie https://www.leitlinienprogrammonkologie.de/leitlinien/, aufgerufen am 06.08.2019

[168] Berwick DM, Hackbarth AD. Eliminating waste in US health care. JAMA 2012; 307: 1513-1516

[169] Raspe H. Die Choosing Wisely Initiative: Hintergründe, Ziele und Probleme einer professionellen Initiative zur Vermeidung von Überversorgung. Z Evid Fortbild Qual Gesundhwes 2017; 129: 12-17

[170] Schöne-Seifert B. Choosing Wisely - Klug Entscheiden: begriffliche und ethische Überlegungen. Z Evid Fortbild Qual Gesundhwes 2017; 129: 41-45

[171] Morden NE, Colla CH, Sequist TD et al. Choosing Wisely - The Politics and Economics of Labeling Low-Value Services. New England Journal of Medicine 2014; 370: 589-592

[172] Klug Entscheiden https://www.klug-entscheiden.com/home/, aufgerufen am 05.08.2019

[173] Mit System zum Ergebnis. Wie entsteht eine „Gemeinsam Klug Entscheiden“-Empfehlung, wer arbeitet daran mit, was sind die Kriterien? https://www.awmf.org/fileadmin/user_upload/Medizinische_Versorgung/GKE/GKE-PM-Berliner\%20Forum\%20AWMF_ Kopp_2015-10-14.pdf, aufgerufen am 19.07.2019

[174] Sackett DL, Rosenberg WMC, Gray JAM et al. Evidence based medicine: what it is and what it isn't. BMJ 1996; 312: 71-72

[175] Leitartikel David Sackett https://www.ebm-netzwerk.de/was-istebm/leitartikel-sackett/, aufgerufen am 06.08.2019

[176] Cochrane Kompakt https://www.cochrane.org/de/evidence, aufgerufen am 06.08.2019

[177] Cochrane Library https://www.cochranelibrary.com/, aufgerufen am 06.08.2019

[178] Deutsches Netzwerk Evidenzbasierte Medizin https://www. ebm-netzwerk.de/, aufgerufen am 06.08.2019

[179] Deutsches Netzwerk Versorgungsforschung https://www.netzwerkversorgungsforschung.de/ aufgerufen am 06.08.2019

[180] SRV-Gutachten 2018 https://www.svr-gesundheit.de/fileadmin/ user_upload/Gutachten/2018/SVR-Gutachten_2018_WEBSEITE.pdf, aufgerufen am 14.08.2019

[181] Stellungnahme Patientenwohl als ethischer Maßstab für das Krankenhaus https://www.ethikrat.org/fileadmin/Publikationen/ Stellungnahmen/deutsch/stellungnahme-patientenwohl-als-ethischer-massstab-fuer-das-krankenhaus.pdf, aufgerufen am 12.08.2019
[182] Wehkamp K-H, Naegler H. Ökonomisierung patientenbezogener Entscheidungen im Krankenhaus. Dtsch Arztebl International 2019; 116: 33

[183] Nimptsch U, Bolczek C, Spoden M et al. Mengenentwicklung stationärer Behandlungen bei Erkrankungen der Wirbelsaule - Analyse der deutschlandweiten Krankenhausabrechnungsdaten von 2005 bis 2014. Z Orthop Unfall 2018; 156: 175-183

[184] Investitionsbedarf der Krankenhäuser: aktuelle Auswertung bestätigt Unterfinanzierung durch die Bundesländer https://www.gkv-spitzenverband.de/media/dokumente/presse/pressemitteilungen/2019/ Gm_PM_2019-03-21_Investitionsbewertungsrelationen_2019.pdf, aufgerufen am 19.07.2019

[185] Positionspapier für eine Reform der medizinischen Notfallversorgung in Deutschland https://www.divi.de/empfehlungen/publikationen/ notfallmedizin/381-positionspapier-fuer-eine-reform-der-med-notfallversorgung-in-deutschland/file aufgerufen am 08.07.2019

[186] Gütekriterien für ein Instrument zur standardisierten Ersteinschätzung von Notfallpatienten https://www.marburger-bund.de/sites/ default/files/files/2019-06/2019_06_06_MB-KBV_Ersteinschätzungsinstrument.pdf, aufgerufen am 08.07.2019

[187] Eine gemeinsame Anlaufstelle für die Akut-und Notfallversorgungin Deutschland https://www.marburger-bund.de/sites/default/files/ files/2019-06/2019_05_23_MB-KBV_Anlaufstellen.pdf, aufgerufen am 08.07.2019

[188] Sammelband Klug Entscheiden 2019 https://www.klug-entscheiden. com/fileadmin/user_upload/2019_Sammelband_Klug_entschieden. pdf, aufgerufen am 06.08.2019

[189] Lyu H, Xu T, Brotman D et al. Overtreatment in the United States. PLOS ONE 2017; 12: e0181970

[190] Neuordnung Krankenhauslandschaft: Weniger ist mehr https://www. bertelsmann-stiftung.de/de/unsere-projekte/krankenhaus-landschaft/projektbeschreibung/ aufgerufen am 22.08.2019

[191] TJC. Physical and verbal violence against health care workers. Sentinel Event Alert 2018; 1-9

[192] Beschlussprotokoll 122. Deutscher Ärztetag https://www.bundesaerztekammer.de/fileadmin/user_upload/downloads/pdf-Ordner/122. DAET/122DAETBeschlussprotokoll.pdf, aufgerufen am 19.08.2019

[193] Lancet. Physician burnout: a global crisis. Lancet 2019; 394: 93

[194] Beschoner P, Limbrecht-Ecklundt K, Jerg-Bretzke L. Psychische Gesundheit von Ärzten : Burnout, Depression, Angst und Substanzgebrauch im Kontext des Arztberufes. Nervenarzt 2019, doi:10.1007/s00115-019-0739-x

[195] Arztgesundheit: Künftig nicht nur eine Floskel https://www. aerzteblatt.de/archiv/208223/Arztgesundheit-Kuenftig-nicht-nureine-Floskel, aufgerufen am 19.08.2019

[196] Sancar F. A Cultural Sea Change: Mindfulness for Surgical ResidentsA Cultural Sea Change: Mindfulness for Surgical ResidentsA Cultural Sea Change: Mindfulness for Surgical Residents. JAMA 2019, doi:10.1001/jama.2019.9999

[197] Topfer A, Brabander G. Personalisierung von Gesundheitsleistungen - Gestaltungsempfehlungen zur Umsetzung. Z Evid Fortbild Qual Gesundhwes 2018; 130: 27-34

[198] Herrera-Perez D, Haslam A, Crain T et al. A comprehensive review of randomized clinical trials in three medical journals reveals 396 medical reversals. eLife 2019; 8: e45183

[199] Goldkuhle M, Jakob T, Kreuzberger $N$ et al. Übertragbarkeit: Ist GRADE die Lösung? Z Evid Fortbild Qual Gesundhwes 2019; 140: 52-57

[200] Gerhardus A, Barbek R. Berücksichtigung des Kontexts bei der Bewertung der Übertragbarkeit von Evidenz. Z Evid Fortbild Qual Gesundhwes 2019; 141-142: 62-65

[201] Antes G. Ist das Zeitalter der Kausalität vorbei? Z Evid Fortbild Qual Gesundhwes 2016; 112 (Suppl 1): S16-22 
[202] Davis C, Lexchin J, Jefferson T et al. "Adaptive pathways" to drug authorisation: adapting to industry? BMJ 2016; 354: i4437

[203] Wolf BR, Buckwalter JA. Randomized surgical trials and "sham" surgery: relevance to modern orthopaedics and minimally invasive surgery. lowa Orthop J 2006; 26: 107-111

[204] Wartolowska K, Judge A, Hopewell S et al. Use of placebo controls in the evaluation of surgery: systematic review. BM] : British Medical Journal 2014; 348: g3253

[205] Thomsen J, Bretlau P, Tos M et al. Meniere's disease: endolymphatic sac decompression compared with sham (placebo) decompression. Ann N Y Acad Sci 1981; 374: 820-830

[206] Thomsen J, Bretlau P, Tos M et al. Placebo effect in surgery for Meniere's disease: three-year follow-up. Otolaryngol Head Neck Surg 1983; 91: 183-186
[207] Gobel P. Zentrenbildung, Register und Qualitätssicherung : Ökonomische Auswirkung auf Ausbildung und Versorgungsqualität. Orthopade 2018; 47: 849-856

[208] Gemeinsame Stellungnahme von Bundesärztekammer und Kassenärztlicher Bundesvereinigung zur Anfrage der Gesundheitsministerkonferenz vom 20.7.98 https://www.aezq.de/mdb/edocs/pdf/stellungnahmen/ sn-qm-1998.pdf, aufgerufen am 15.07.2019

[209] Sanrivus Healthcare Consulting www.sanrivus.de aufgerufen am 26.08.2019

[210] Beck M. Can a Death-Predicting Algorithm Improve Care? The Wall Street Journal. 02.12.2016

[211] Avati A, Jung K, Harman S et al. Improving palliative care with deep learning. BMC Med Inform Decis Mak 2018; 18: 122 